OPEN ACCESS

\section{A method to determine the electric field of liquid argon time projection chambers using a UV laser system and its application in MicroBooNE}

To cite this article: C. Adams et al 2020 JINST 15 P07010

View the article online for updates and enhancements.

\section{Recent citations}

$$
\begin{aligned}
& \text { - Convolutional neural network for multiple } \\
& \frac{\text { particle identification in the MicroBooNE }}{\text { liquid argon time projection chamber }} \\
& \text { P. Abratenko et al } \\
& \text { - Measurement of the atmospheric muon } \\
& \frac{\text { rate with the MicroBooNE Liquid Arqon }}{\text { TPC }} \\
& \text { - Abratenko et al } \\
& \frac{\text { Vertex-finding and reconstruction of }}{\text { MicroBoontained two-track neutrino events in the }} \\
& \text { P.Abratenko et al }
\end{aligned}
$$

The goal of ECS Sensors Plus, as a one-stop shop journal for sensors, is to advance the fundamental science and understanding of sensors and detection technologies for efficient monitoring and control of industrial processes and the environment, and improving quality of life and human health.

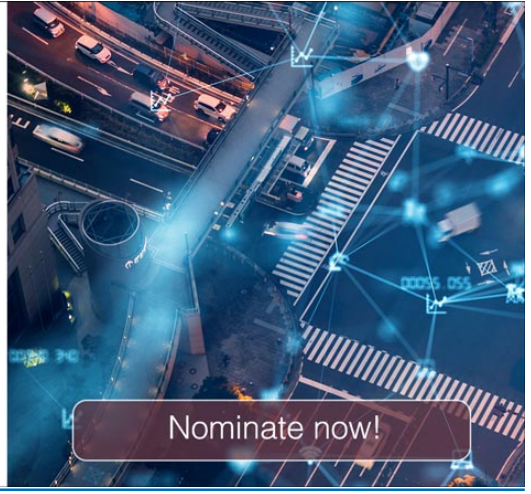




\section{A method to determine the electric field of liquid argon time projection chambers using a UV laser system and its application in MicroBooNE}

\section{MicroBooNE Collaboration}

C. Adams, ${ }^{k}$ M. Alrashed, ${ }^{m}$ R. An, ${ }^{l}$ J. Anthony, ${ }^{d}$ J. Asaadi, ${ }^{d d}$ A. Ashkenazi, ${ }^{q}$

S. Balasubramanian, ${ }^{i i}$ B. Baller, ${ }^{j}$ C. Barnes, ${ }^{r}$ G. Barr, ${ }^{u}$ V. Basque, ${ }^{p}$ M. Bass, ${ }^{b}$ F. Bay, ${ }^{e e}$

S. Berkman, ${ }^{j}$ A. Bhanderi, ${ }^{p}$ A. Bhat, ${ }^{a}{ }^{a}$ M. Bishai, ${ }^{b}$ A. Blake, ${ }^{n}$ T. Bolton, ${ }^{m}$ L. Camilleri, ${ }^{h}$

D. Caratelli, ${ }^{j}$ I. Caro Terrazas, ${ }^{g}$ R. Carr, ${ }^{q}$ R. Castillo Fernandez, ${ }^{j}$ F. Cavanna, ${ }^{j}$ G. Cerati, ${ }^{j}$

Y. Chen, ${ }^{a}$ E. Church, ${ }^{v}$ D. Cianci, ${ }^{h}$ E.O. Cohen, ${ }^{b b}$ J.M. Conrad, ${ }^{q}$ M. Convery, ${ }^{y}$

L. Cooper-Troendle, ${ }^{i}$ J.I. Crespo-Anadón, ${ }^{h}$ M. Del Tutto, ${ }^{u, k}$ D. Devitt, ${ }^{n}$ A. Diaz, ${ }^{q}$ L. Domine, ${ }^{y}$

K. Duffy, ${ }^{j}$ S. Dytman, ${ }^{w}$ B. Eberly, ${ }^{i}$ A. Ereditato, ${ }^{a}$ L. Escudero Sanchez, ${ }^{d}$ J.J. Evans, ${ }^{p}$

R.S. Fitzpatrick, ${ }^{r}$ B.T. Fleming, ${ }^{i i}$ N. Foppiani, ${ }^{k}$ D. Franco, ${ }^{i i}$ A.P. Furmanski, ${ }^{p}$

D. Garcia-Gamez, ${ }^{p}$ S. Gardiner, ${ }^{j}$ V. Genty, ${ }^{h}$ D. Goeldi, ${ }^{a}$ S. Gollapinni, ${ }^{c c}$ O. Goodwin, ${ }^{p}$

E. Gramellini, ${ }^{j}$ P. Green, ${ }^{p}$ H. Greenlee,${ }^{j}$ R. Grosso,${ }^{f}$ L. Gu, ${ }^{g g}$ W. Gu, ${ }^{b}$ R. Guenette, ${ }^{k}$

P. Guzowski, ${ }^{p}$ P. Hamilton, ${ }^{a}{ }^{a}$ O. Hen, ${ }^{q}$ C. Hill, ${ }^{p}$ G.A. Horton-Smith, ${ }^{m}$ A. Hourlier, ${ }^{q}$

E.-C. Huang, ${ }^{o}$ R. Itay, ${ }^{y}$ C. James, ${ }^{j}$ J. Jan de Vries, ${ }^{d}$ X. Ji, ${ }^{b}$ L. Jiang, ${ }^{w}$ J.H. Jo, ${ }^{i i}$

R.A. Johnson, ${ }^{f}$ J. Joshi, ${ }^{b}$ Y.-J. Jwa, ${ }^{h}$ G. Karagiorgi, ${ }^{h}$ W. Ketchum, ${ }^{j}$ B. Kirby, ${ }^{b}$ M. Kirby, ${ }^{j}$

T. Kobilarcik, ${ }^{j}$ I. Kreslo, ${ }^{a}$ I. Lepetic, ${ }^{l}$ Y. Li, ${ }^{b}$ A. Lister, ${ }^{n}$ B.R. Littlejohn, ${ }^{l}$ S. Lockwitz, ${ }^{j}$

D. Lorca, ${ }^{a}$ W.C. Louis, ${ }^{o}$ M. Luethi, ${ }^{a}$ B. Lundberg, ${ }^{j}$ X. Luo, ${ }^{i i, c}$ A. Marchionni, ${ }^{j}$ S. Marcocci, ${ }^{j}$

C. Mariani, ${ }^{g g}$ J. Marshall, ${ }^{h} h$ J. Martin-Albo, ${ }^{k}$ D.A. Martinez Caicedo, ${ }^{z}$ K. Mason, ${ }^{f f}$

A. Mastbaum, ${ }^{e}$ N. McConkey, ${ }^{p}$ V. Meddage, ${ }^{m}$ T. Mettler, ${ }^{a}$ K. Miller, ${ }^{e}$ J. Mills, ${ }^{f f}$ K. Mistry, ${ }^{p}$

A. Mogan, ${ }^{c c}$ T. Mohayai, ${ }^{j}$ J. Moon, ${ }^{q}$ M. Mooney, ${ }^{g}$ C.D. Moore ${ }^{j}$ J. Mousseau, ${ }^{r}$ M. Murphy, ${ }^{g g}$

R. Murrells, ${ }^{p}$ D. Naples, ${ }^{w}$ R.K. Neely, ${ }^{m}$ P. Nienaber, ${ }^{x}$ J. Nowak, ${ }^{n}$ O. Palamara, ${ }^{j}$ V. Pandey, ${ }^{g g}$

V. Paolone, ${ }^{w}$ A. Papadopoulou, ${ }^{q}$ V. Papavassiliou, ${ }^{s}$ S.F. Pate,${ }^{s}$ A. Paudel, ${ }^{m}$ Z. Pavlovic, ${ }^{j}$

E. Piasetzky, ${ }^{b b}$ D. Porzio, ${ }^{p}$ S. Prince, ${ }^{k}$ G. Pulliam, ${ }^{a} a$ X. Qian, ${ }^{b}$ J.L. Raaf, ${ }^{j}$ V. Radeka, ${ }^{b}$

A. Rafique, ${ }^{m}$ L. Ren, ${ }^{s}$ L. Rochester, ${ }^{y}$ H.E. Rogers, ${ }^{g}$ M. Ross-Lonergan, ${ }^{h}$ C. Rudolf von Rohr, ${ }^{a}$

B. Russell, ${ }^{i i}$ G. Scanavini, ${ }^{i i}$ D.W. Schmitz, ${ }^{e}$ A. Schukraft, ${ }^{j}$ W. Seligman, ${ }^{h}$ M.H. Shaevitz, ${ }^{h}$

R. Sharankova, ${ }^{f f}$ J. Sinclair, ${ }^{a}$ A. Smith, ${ }^{d}$ E.L. Snider, ${ }^{j}$ M. Soderberg, ${ }^{a}{ }^{a}$

S. Söldner-Rembold, ${ }^{p}$ S.R. Soleti, ${ }^{u, k}$ P. Spentzouris, ${ }^{j}$ J. Spitz, ${ }^{r}$ M. Stancari, ${ }^{j}$ J. St. John, ${ }^{j}$

T. Strauss, ${ }^{j}$ K. Sutton, ${ }^{h}$ S. Sword-Fehlberg, ${ }^{s}$ A.M. Szelc, ${ }^{p}$ N. Tagg, ${ }^{t}$ W. Tang, $^{c c}$ K. Terao, ${ }^{y}$

R.T. Thornton, ${ }^{o}$ M. Toups, ${ }^{j}$ Y.-T. Tsai, ${ }^{y}$ S. Tufanli, ${ }^{i i}$ M.A. Uchida, ${ }^{d}$ T. Usher, ${ }^{y}$

W. Van De Pontseele, ${ }^{u, k}$ R.G. Van de Water, ${ }^{o}$ B. Viren, ${ }^{b}$ M. Weber, ${ }^{a}$ H. Wei, ${ }^{b}$

D.A. Wickremasinghe, ${ }^{w}$ Z. Williams, ${ }^{d d}$ S. Wolbers, ${ }^{j}$ T. Wongjirad, ${ }^{f f}$ K. Woodruff, ${ }^{s}$ 


\section{Wospakrik, ${ }^{j}$ W. Wu, ${ }^{j}$ T. Yang, ${ }^{j}$ G. Yarbrough, ${ }^{c c}$ L.E. Yates, ${ }^{q}$ G.P. Zeller, ${ }^{j}$ J. Zennamo ${ }^{j}$ and C. Zhang ${ }^{b}$}

${ }^{a}$ Universität Bern, Bern CH-3012, Switzerland

${ }^{b}$ Brookhaven National Laboratory (BNL), Upton, NY, 11973, U.S.A.

${ }^{c}$ University of California, Santa Barbara, CA, 93106, U.S.A.

${ }^{d}$ University of Cambridge, Cambridge CB3 OHE, United Kingdom

${ }^{e}$ University of Chicago, Chicago, IL, 60637, U.S.A.

${ }^{f}$ University of Cincinnati, Cincinnati, OH, 45221, U.S.A.

${ }^{g}$ Colorado State University, Fort Collins, CO, 80523, U.S.A.

${ }^{h}$ Columbia University, New York, NY, 10027, U.S.A.

${ }^{i}$ Davidson College, Davidson, NC, 28035, U.S.A.

${ }^{j}$ Fermi National Accelerator Laboratory (FNAL), Batavia, IL 60510, U.S.A.

${ }^{k}$ Harvard University, Cambridge, MA 02138, U.S.A.

${ }^{l}$ Illinois Institute of Technology (IIT), Chicago, IL 60616, U.S.A.

${ }^{m}$ Kansas State University (KSU), Manhattan, KS, 66506, U.S.A.

${ }^{n}$ Lancaster University, Lancaster LA1 4YW, United Kingdom

${ }^{o}$ Los Alamos National Laboratory (LANL), Los Alamos, NM, 87545, U.S.A.

${ }^{p}$ The University of Manchester, Manchester M13 9PL, United Kingdom

${ }^{q}$ Massachusetts Institute of Technology (MIT), Cambridge, MA, 02139, U.S.A.

${ }^{r}$ University of Michigan, Ann Arbor, MI, 48109, U.S.A.

${ }^{s}$ New Mexico State University (NMSU), Las Cruces, NM, 88003, U.S.A.

${ }^{t}$ Otterbein University, Westerville, OH, 43081, U.S.A.

${ }^{u}$ University of Oxford, Oxford OX1 3RH, United Kingdom

${ }^{v}$ Pacific Northwest National Laboratory (PNNL), Richland, WA, 99352, U.S.A.

${ }^{w}$ University of Pittsburgh, Pittsburgh, PA, 15260, U.S.A.

${ }^{x}$ Saint Mary's University of Minnesota, Winona, MN, 55987, U.S.A.

${ }^{y}$ SLAC National Accelerator Laboratory, Menlo Park, CA, 94025, U.S.A.

${ }^{z}$ South Dakota School of Mines and Technology (SDSMT), Rapid City, SD, 57701, U.S.A.

${ }^{a}$ Syracuse University, Syracuse, NY, 13244, U.S.A.

${ }^{b b}$ Tel Aviv University, Tel Aviv, 69978, Israel

cc University of Tennessee, Knoxville, TN, 37996, U.S.A.

${ }^{d d}$ University of Texas, Arlington, TX, 76019, U.S.A.

ee TUBITAK Space Technologies Research Institute, METU Campus, TR-06800, Ankara, Turkey

${ }^{f f}$ Tufts University, Medford, MA, 02155, U.S.A.

${ }^{g g}$ Center for Neutrino Physics, Virginia Tech, Blacksburg, VA, 24061, U.S.A.

${ }^{\text {hh } U n i v e r s i t y ~ o f ~ W a r w i c k, ~ C o v e n t r y ~ C V 4 ~ 7 A L, ~ U n i t e d ~ K i n g d o m ~}$

${ }^{i i}$ Wright Laboratory, Department of Physics, Yale University, New Haven, CT, 06520, U.S.A.

E-mail: microboone_info@fnal.gov

AвSTRACT: Liquid argon time projection chambers (LArTPCs) are now a standard detector technology for making accelerator neutrino measurements, due to their high material density, precise tracking, and calorimetric capabilities. An electric field (E-field) is required in such detectors to 
drift ionization electrons to the anode where they are collected. The E-field of a TPC is often approximated to be uniform between the anode and the cathode planes. However, significant distortions can appear from effects such as mechanical deformations, electrode failures, or the accumulation of space charge generated by cosmic rays. The latter effect is particularly relevant for detectors placed near the Earth's surface and with large drift distances and long drift time. To determine the E-field in situ, an ultraviolet (UV) laser system is installed in the MicroBooNE experiment at Fermi National Accelerator Laboratory. The purpose of this system is to provide precise measurements of the E-field, and to make it possible to correct for 3D spatial distortions due to E-field non-uniformities. Here we describe the methodology developed for deriving spatial distortions, the drift velocity and the E-field from UV-laser measurements.

Keywords: Ionization and excitation processes; Neutrino detectors; Noble liquid detectors (scintillation, ionization, double-phase); Time projection chambers 


\section{Contents}

1 Introduction 1

2 Laser system description and TPC volume scans 2

2.1 Laser scan 2

2.2 True laser track position 4

$\begin{array}{lll}3 & \text { Reconstruction and selection of laser tracks } & 7\end{array}$

3.1 Hit and track reconstruction 7

3.2 Laser track selection 8

4 Laser track simulations $\quad 8$

4.1 Toy simulation 9

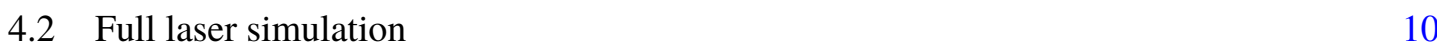

5 Spatial displacement map $\quad 10$

$\begin{array}{lll}5.1 & \text { Spatial displacement vectors } & 11\end{array}$

$\begin{array}{lll}\text { 5.1.1 Closest-point projection } & 11\end{array}$

$\begin{array}{lll}\text { 5.1.2 Track iteration } & 12\end{array}$

$\begin{array}{lll}5.2 & \text { Boundary condition } & 13\end{array}$

$\begin{array}{lll}5.3 & \text { Interpolation } & 13\end{array}$

$\begin{array}{lll}5.4 & \text { Sub-map merging } & 15\end{array}$

$\begin{array}{lll}5.5 & \text { Bias study } & 16\end{array}$

$\begin{array}{lll}\text { 5.5.1 Bias study using toy simulation } & 16\end{array}$

$\begin{array}{ll}\text { 5.5.2 Bias study using full laser simulation } & 17\end{array}$

5.5.3 Coverage of the TPC volume with the UV-laser method 18

$6 \quad$ E-field and drift velocity maps $\quad 24$

$\begin{array}{lll}6.1 & \text { Field lines } & 24\end{array}$

$\begin{array}{lll}6.2 & \text { Extraction of the local drift velocity } & 27\end{array}$

6.3 Extraction of the local E-field 27

6.4 Validation of the E-field determination 28

7 Measurements in MicroBooNE from laser data 29

$\begin{array}{lll}7.1 & \text { Distortion maps } & 29\end{array}$

7.2 Drift velocity map from laser data 33

7.3 E-field map measured from laser data 34

8 Time stability studies $\quad 35$

9 Conclusion $\quad 41$ 


\section{Introduction}

Liquid argon time projection chambers (LArTPCs) are excellent detectors for neutrino experiments at many scales, through very massive detectors. Several massive LArTPCs have already been built and the technology will also be used for the Deep Underground Neutrino Experiment (DUNE) [1]. MicroBooNE [2] is the first large LArTPC with $85 \mathrm{t}$ active mass built at the Fermi National Accelerator Laboratory (FNAL) as part of the short baseline neutrino (SBN) program [3]. As the MicroBooNE detector is placed near the earths' surface, space charge effects induced by comparatively slow drifting ions produced by cosmic ray muons can alter the local electric field (E-field). Since the ion drift speeds are $\sim 10^{5}$ slower than the electron drift speeds in the same E-field [4-6] and are comparable to fluid speeds generated by convection in the cryostat [2], space charge effects are difficult to predict. These E-field variations can adversely affect event reconstruction, as they cause ionization electrons to experience different drift velocities along their path to the anode and to have different recombination rates at where they are produced. This reduces track reconstruction efficiencies of the detector and introduces additional systematic uncertainties in energy reconstruction. To account for these spatial distortions and measure the E-field together with the drift velocity in MicroBooNE, a novel ultraviolet (UV) laser calibration system has been installed. Unlike cosmic muons, another calibration source, laser beams do not experience delta ray emission or multiple Coulomb scattering in LAr. Laser beams can also be repetitively pulsed in controllable directions. Additionally, the UV laser system can be used to investigate detector failures, such as unresponsive or mis-configured wires in the read-out planes.

It has been shown that a UV laser can generate tracks in a LArTPC through multiphoton ionization [7-10]. Reproducible laser tracks over $5 \mathrm{~m}$ long are observed in ARGONTUBE [11]. The attenuation of the laser beam pulse energy is negligible at this scale. The feasibility of using a UV laser for free electron lifetime measurements in LArTPCs has also been shown [11]. Multiphoton ionization strongly depends on the laser beam intensity. Argon atoms in the liquid phase can be lifted to an excited state through a laser-induced virtual state by absorption of two UV photons $(266 \mathrm{~nm})$. An additional UV photon provides the correct energy to ionize the excited argon atom in liquid phase. A more detailed discussion of this process can be found in ref. [10].

In section 2, we describe the hardware set-up of the MicroBooNE laser system, its operation, and the result of laser beams scanning over the TPC volume and the calculation of true laser track positions, which are the actual paths of laser beams. The track reconstruction and selection are both optimized for the laser, which as is illustrated in section 3. A simulation of laser tracks is described in section 4. In section 5, we describe a complete methodology for computing track point spatial displacement maps and their uncertainties using reconstructed laser tracks and their true positions (straight lines). A bias study of the methodology is demonstrated using laser track simulations in the same section. Techniques for extracting the E-field and drift velocity over the TPC volume from the spatial displacement maps are explained in section 6. The measurement results of MicroBooNE spatial displacement, drift velocity and E-field maps are presented in section 7. A study of the temporal stability of the E-field is shown in section 8, with the distortion measured continuously over several hours. 


\section{Laser system description and TPC volume scans}

The MicroBooNE laser system consists of two identical UV laser sub-systems. One is located upstream of the TPC with respect to the Booster neutrino beam, and the other is located downstream of the TPC. Each sub-system uses a commercial Nd:YAG laser module Surelite I-10 [12]. The Surelite I-10 initially generates infrared (IR) light $(1064 \mathrm{~nm})$, which is shifted to green $(532 \mathrm{~nm})$ first, and then to UV $(266 \mathrm{~nm})$ through second and fourth harmonic generators. The output laser light has most of its intensity in the UV, with significant residual green and IR. The maximum laser pulse repetition rate is $10 \mathrm{~Hz}$. The pulse duration is $4 \mathrm{~ns}$ to $6 \mathrm{~ns}$. The UV laser light output of the Surelite I-10 in each pulse has an energy of $60 \mathrm{~mJ}$.

To select only UV light, we use a wavelength separator which is composed of two dichroic mirrors, an attenuator, and an aperture. Figure 1 shows a schematic of the laser systems. The first mirror (M1) has high transmittance for IR light and high reflectance for green and UV light. A beam dump (BD1) at the transmitting side of M1 stops the IR laser light. The second mirror (M2) has high reflectance for UV light while most of the green light is transmitted, terminating at another beam dump (BD3). M2 is mounted on a ZABER T-OMG [13]. The gimbal has actuators which provide remote control. Both dichroic mirrors are supplied by Continuum [14]. In between M1 and M2, there is an Altechna Enhanced Watt Pilot attenuator [15, 16], which is operating in transmission mode. The attenuation is motorized and can be controlled remotely, with a range of $0.5 \%$ to $95 \%$ transmittance at $266 \mathrm{~nm}$. It is used to lower and stabilize the laser beam pulse energy. If the energy of the laser pulse is too high or too low, there will be too many or too few ionization electrons to leave a well defined track. A beam dump (BD2) is at the backside of the attenuator to absorb the passing laser. In front of M2, a remote-controlled aperture is used to limit the diameter of the transverse laser beam to $1 \mathrm{~mm}$.

A green laser is placed at the backside of M1 in order to reach the same optical path as the UV laser beam. It can give a visual guide for the UV laser beam during alignment and maintenance. A photodiode is used to trigger the data acquisition (DAQ) for laser events when its signal crosses a threshold. Thus, each laser event contains one laser track, unless the beam pulse is blocked by the field-cage rings (figure 4), which are used to shape E-field of the TPC along the drift distance.

All the optical components mentioned above are contained within a light-tight aluminum "laser box". The output of the laser box is the UV-only beam which is guided to the last warm mirror (M3). It uses the same gimbal as M2, enabling remote control of the mirror angle. The last mirror (cold mirror) is in liquid argon and reflects the UV-laser beam into the TPC volume. It is supported by a $2.5 \mathrm{~m}$-long feed-through. The cold mirror is controlled by two motors [17], and can rotate both in the $Y-Z$ and $X-Z$ plane. Two independent encoders measure the azimuthal and polar angles with high precision. A $2 \mathrm{~m}$-long evacuated quartz light guide allows the UV-laser beam to enter the LAr without disturbance at the liquid surface. The cold mirror is within the cryostat but outside of the TPC. It is mounted close to the field-cage rings, which block some beam paths into the TPC. A detailed description of the laser system can be found in refs. [2] and [7].

\subsection{Laser scan}

We use the MicroBooNE coordinate system where the $X$ coordinate is the drift direction, with the anode (readout plane) at $X=0 \mathrm{~cm}$, and the cathode at $X=254.4 \mathrm{~cm}$; The $Y$ coordinate is vertical 


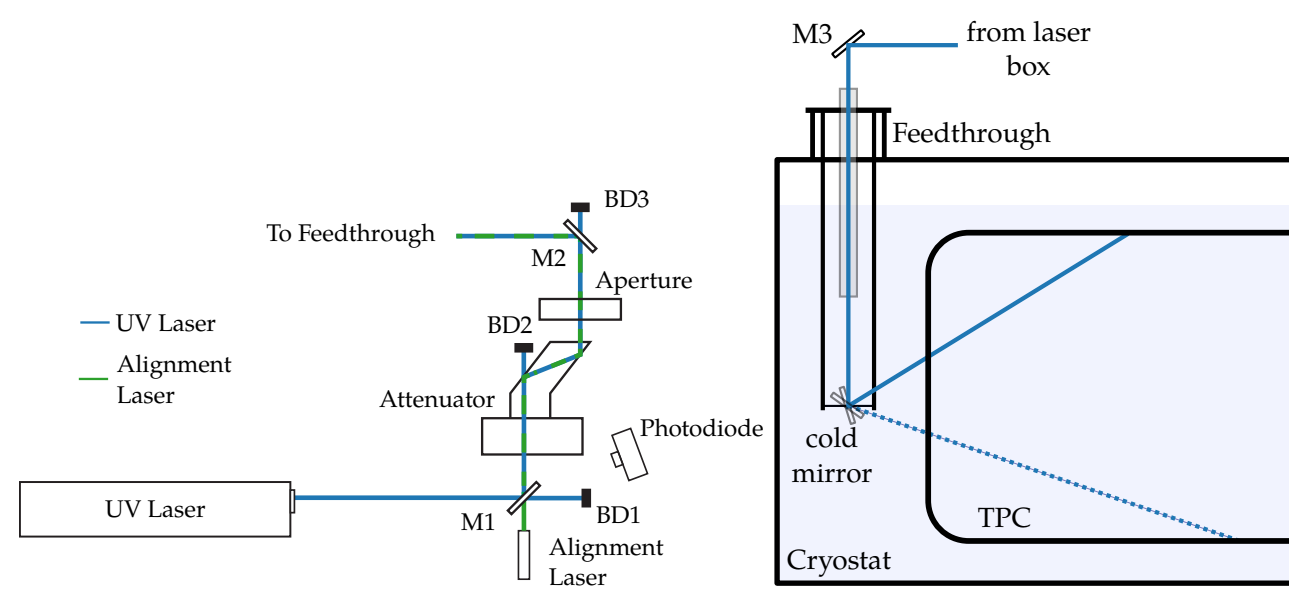

Figure 1. The laser system of MicroBooNE; the upstream and downstream laser sub-systems have the same layout. Left: The optical path of the laser beam before entering the cryostat. The $266 \mathrm{~nm}$ laser beam is aligned by two mirrors (M1 and M2), and directed towards the feedthrough. An attenuator for beam energy control and an aperture for beam size control are placed in between the two mirrors M1 and M2. A photodiode provides a trigger for the readout. Right: Schematic of the laser feedthrough in the cryostat. The UV laser beam reflects at the dichroic mirror M3 and then enters an evacuated quartz tube which serves as light guide to avoid defocusing of the laser beam at the LAr surface. The cold mirror can rotate horizontally together with the feedthrough assembly. A movable rod extending to a cogwheel allows the cold mirror to rotate vertically. The supporting structure of the cold mirror is mounted to the feedthrough flange.

with a range from $-116.25 \mathrm{~cm}$ to $116.25 \mathrm{~cm}$, from the bottom to the top of both the cathode and the anode planes; The $Z$ coordinate is along the beam direction, where $Z=0 \mathrm{~cm}$ is at the upstream end of the TPC, closest to the neutrino source, and $Z=1036.8 \mathrm{~cm}$ is at the downstream end. These TPC boundaries are the physical limits of the true spatial coordinates. This is a right-handed coordinate system, and the anode plane is to the right when looking downstream in $Z$. Figure 2 demonstrates the size of the TPC active volume and the coordinate system.

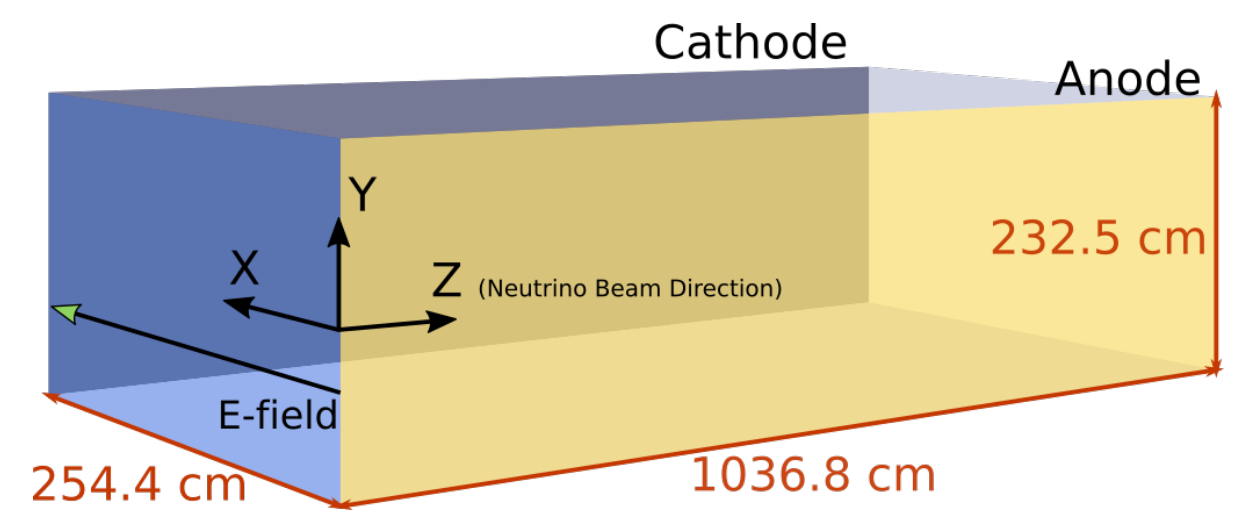

Figure 2. The figure shows the size of the TPC active volume and the coordinate system. The Booster neutrino beam is along $Z$ direction.

A full laser scan consists of a range of measurements from both laser sub-systems. The top view ( $X-Z$ projection) of a full laser scan pattern with reconstructed laser tracks can be seen in figure 3. Field-cage rings located between the cold mirror and the TPC volume obstruct part 
of the laser beam paths, which results in gaps in the laser scan. The TPC corners close to the cathode at the upstream and the downstream ends are not accessible because of the field-cage rings. Laser beams are not aimed in the direction of the anode since photomultiplier tubes (PMTs) and corresponding wavelength shifting plates are mounted behind the anode. To maintain the efficiency of the wavelength shifting plates installed in front of the PMTs, exposure to intense UV light should be avoided. In a laboratory measurement at the University of Bern, the effect of the beam of the same Surelite UV-laser as employed in MicroBooNE was tested on a wavelength plate coated with tetraphenyl butadiene (TPB). Its effect was found to be negligible. To obtain a noticeable degradation, the full TPB area (5 $\mathrm{cm}$ in diameter) had to be directly illuminated at full laser pulse power. Even in this unlikely scenario, only a $20 \%$ decrease in light conversion was observed after more than 14,000 UV-laser pulses.

In MicroBooNE, the laser scan coverage from both sides is almost symmetric. Slight differences arise from small variations in the attenuator settings between the two sub-systems. In the case of the upstream laser, the laser beams are aimed from $30^{\circ}$ to $140^{\circ}$ with respect to the $Y$ axis. The mechanics of the feedthrough and characteristics of the dichroic mirror limit the range of accessible vertical angle. Horizontally, the laser beams sweep from $45^{\circ}$ to $93^{\circ}$ with respect to the $X$ axis. Smaller angles with respect to $X$ are obscured by the field-cage rings. Larger angles are limited by the field-cage rings and the need to avoid the anode.

To achieve a dense scan pattern and to minimize the E-field distortions from the ions induced by the laser beam itself, the pulse repetition rates are limited to $4 \mathrm{~Hz}$. The laser pulses continually while the cold mirrors move. At the beginning of a scan, a laser sub-system is set to an extreme of both horizontal and vertical ranges. Then a horizontal sweep is started with a speed set to 2000 micro-steps per second $\left(\sim 0.16^{\circ} \mathrm{s}^{-1}\right)$. When the horizontal sweep is complete, the cold mirror tilts vertically without the laser pulsing, and another horizontal sweep in the opposite direction is started. The attenuator setting is updated based on the laser beam incident angle with respect to the cold mirror, to obtain an appropriate laser beam pulse energy. The cold mirror is a dichroic mirror which has different reflectance at different angles of incidence.

Every time the laser pulses, the photo diode triggers an event. Meanwhile, the position of the cold mirror is read out by two encoders and stored on a local server. Data fragments from the laser DAQ and the TPC DAQ are merged offline based on the coincidence of their time stamps. Both of their DAQ time clocks are provided by a common network time protocol (NTP) server.

\subsection{True laser track position}

True laser tracks represent the paths of laser beams in the TPC that are equivalent to the intrinsically straight laser tracks without any E-field distortions. In the TPC, laser tracks are similar to tracks from charged particles. Ionization electrons from both types of tracks drift in the same E-field. Reconstruction of ionization electrons from a given position in the TPC always results in the same fixed read-out position, thus allowing laser tracks to be used to calibrate distortions in the TPC related to the E-field.

True laser track positions are provided by the reflection point on the cold mirror and the angles at which the laser beams cross the TPC. The reflection point and crossing angle provide laser entry and exit points of the TPC. 


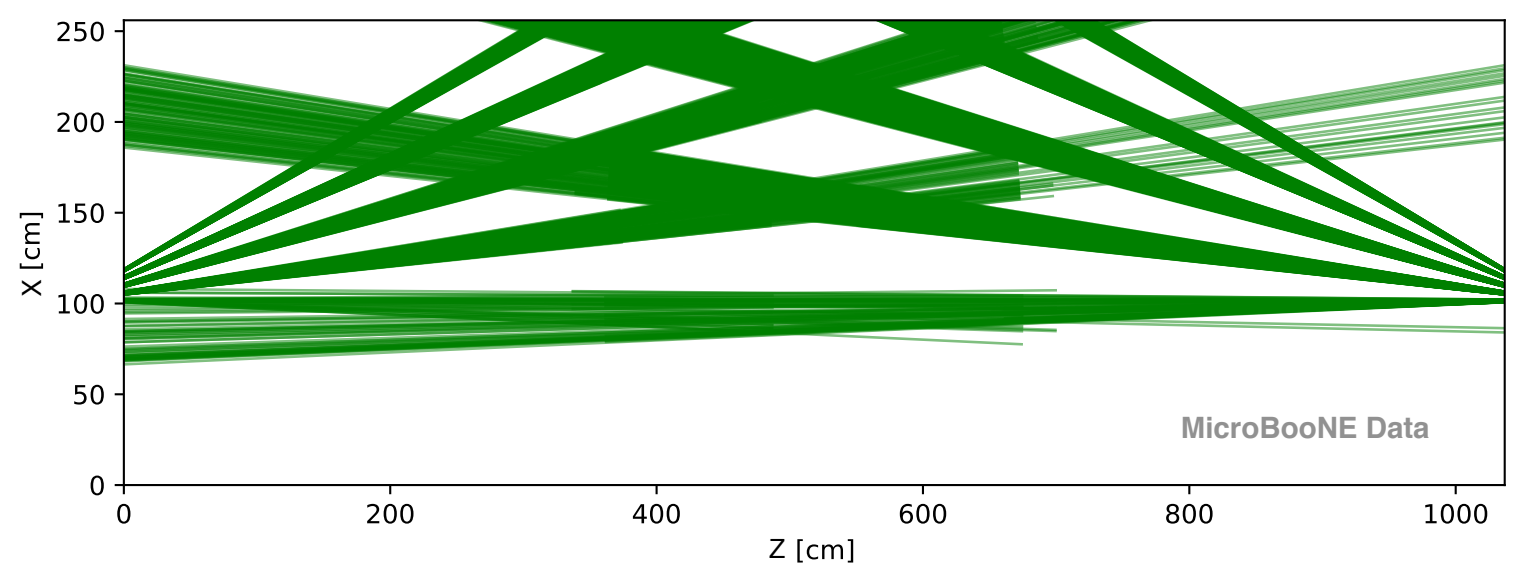

Figure 3. Top view ( $X-Z$ projection) of reconstructed laser tracks (green lines) for both laser sub-systems in the TPC active volume. The laser beams are sent into the TPC from the upstream end (left) and the downstream end (right). Each colored 'fan' is composed of several discrete laser tracks. The laser tracks shown in the plot pass the selection criteria described in section 3.2. Some tracks look like they end because the corresponding laser light exits the TPC at the top and bottom surfaces. Gaps in the coverage arise due to the presence of field-cage rings in front of the cold mirror.

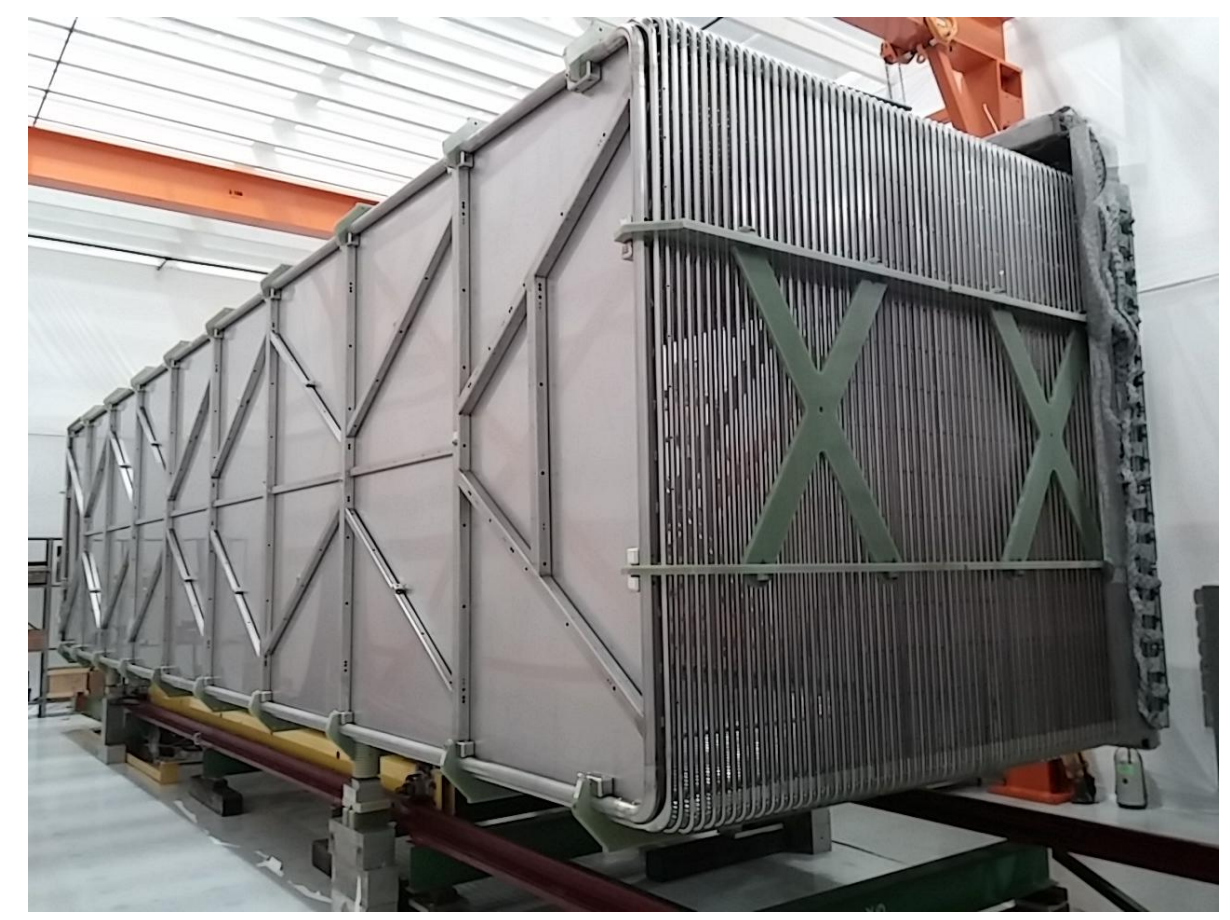

Figure 4. Exterior view of the MicroBooNE time projection chamber showing the cathode frame, structural supports, and the field-cage, before it was placed in the cryostat. There are 63 field-cage rings supported by horizontal G10 beams. Taken from ref. [2]. 
The precise position of the reflection point is determined by measuring the ionization pattern caused by the shadow that the field-cage rings cast on the laser scan. This procedure is illustrated in figure 5. From the engineering drawings, the location of the cold mirror is known to a precision of $O(1 \mathrm{~cm})$, which provides a starting point for the fit of its exact position. In MicroBooNE, there are 63 field-cage rings supported by horizontal G10 beams [2], as shown in figure 4. A slow horizontal scan is applied, producing a dense scan pattern. From this pattern, the sharp edges of the field-cage rings and their separations are apparent. A 2-D $(X-Z)$ fit then determines the $X$ and $Z$ positions of the laser reflection point on the cold mirror. The vertical position $(Y)$ is determined using the same technique with a 1-D fit of the shadow of the horizontal G10 beams. The calibrated positions of the laser reflection points are listed in table 1.

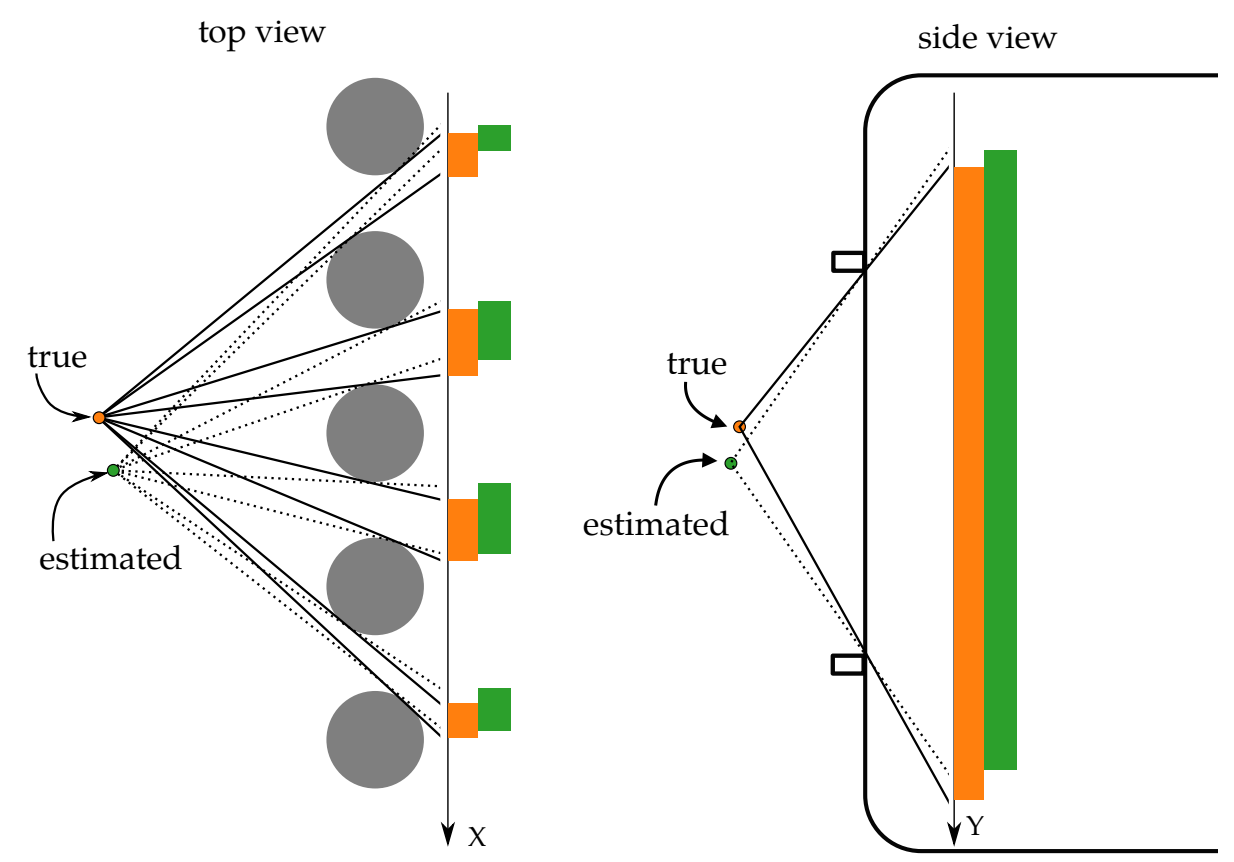

Figure 5. Schematic of the calibration procedure used to determine the position of the laser reflection point on the cold mirror. Left: Top view near the cold mirror showing the determination of the laser reflection point on the cold mirror horizontally in the $X-Z$ plane. The gray circles are the cross-section of the field-cage rings. A horizontal scan produces a pattern of laser ionization delimited by field-cage rings in the TPC represented by the orange boxes at the TPC edge. Any different reflection point in the $X-Z$ plane would leave a different pattern in the horizontal scan, for example, the green boxes. By fitting the $X$ and $Z$ coordinates of the reflection point, the position of the reflection point on the cold mirror is determined. Right: Side view next to the cold mirror showing the determination of the laser reflection point on the cold mirror in $Y$. The two small boxes on the left hand side with solid lines are the G10 beams. A vertical laser scan leaves an ionization pattern delimited by the G10 beams in the TPC represented by the orange (green) bar for the orange (green) beam reflection origin. A fit to the pattern reveals the true reflection point coordinate in $Y$. The determination of the laser reflection point follows the order of horizontal coordinates $(X$ and $Z)$ first, and then the vertical coordinate $(Y)$.

The angles of the laser beams are deduced from the cold mirror positions. As described previously, the cold mirror angles (azimuth and polar) are measured by two independent encoders to a high precision. The position accuracy of true laser tracks is $O(1 \mathrm{~mm})$ at the full distance of $10 \mathrm{~m}$ in the TPC. 
Table 1. Coordinates of the laser reflection point on the cold mirror of the upstream and downstream laser sub-systems, with uncertainties.

\begin{tabular}{|c|c|c|c|}
\hline Sub-system & $X[\mathrm{~cm}]$ & $Y[\mathrm{~cm}]$ & $Z[\mathrm{~cm}]$ \\
\hline Upstream & $103.8 \pm 0.1$ & $8.6 \pm 0.1$ & $-35.6 \pm 0.1$ \\
Downstream & $102.5 \pm 0.1$ & $8.2 \pm 0.1$ & $1080.2 \pm 0.1$ \\
\hline
\end{tabular}

\section{Reconstruction and selection of laser tracks}

\subsection{Hit and track reconstruction}

The MicroBooNE charge readout consists of two induction-wire planes and one collection-wire plane. The collection wires are vertical and wires of the two induction planes are angled at $\pm 60^{\circ}$ with respect to the collection wires. For all three planes, the wire pitch is $3 \mathrm{~mm}$. The read-out wires are indexed by wire IDs. Raw signals are waveforms on each wire with respect to the drift time; details of signal processing are described in refs. [18, 19]. The hit reconstruction identifies hits as the peak amplitudes and times of waveforms associated to wire IDs. The track reconstruction then employs the hits as an input, grouping them to form 3D space points. Reconstructed track objects are a sequence of 3D points with spatial coordinates in $X, Y$ and $Z$.

Hit and track reconstruction for the laser employs LArSoft [20], the standard software toolkit for simulation and reconstruction used in MicroBooNE.

The laser reconstruction procedure first defines a region of interest (ROI) around the true laser track wire IDs and drift time, in order to reduce computing time. Given a spatial position in the TPC, LArSoft provides the expected wire ID for each plane. Thus, a range of wire IDs along the true laser track can be identified. The ROI is extended by \pm 100 wires $(30 \mathrm{~cm})$ around each wire to account for E-field distortions. Similarly, the ROI along the drift axis (time) is also determined by the $X$ coordinates of the true laser track, and extended by $\pm 20 \mathrm{~cm}$. For simplicity, the extensions to the ROI for both wires and drift time remain the same across all events.

A hit contains information about the waveform peak amplitude, width, time, and the associated uncertainties. Typical signals in the collection plane are a single positive peak, whereas signals in induction planes are bipolar peaks. After a baseline correction, a hit is recognized if the waveform exceeds threshold. In the collection plane, the time of the highest peak amplitude is defined as the hit time. In the induction planes, the average of the two bipolar peak times is taken as the hit time. For a laser track, the charge deposition is large and the waveform is broadened to a less well-defined peak. In this case, the local center of the peak is taken as the hit time. If the ratio of height to width is too low, the waveform is not reconstructed as a hit. This hit reconstruction algorithm was developed specifically for laser track reconstruction. The conventional hit reconstruction involves waveform deconvolution which has difficulties with the large charge deposition of laser tracks. Minimum ionizing particles have narrower and shorter waveforms than laser waveform signals which typically are three times larger.

The laser tracks are reconstructed by Pandora [21] tools embedded in LArSoft using the collection of hits as an input. The tracks are groups of 3D space points which have separate primary ionization origins. 


\subsection{Laser track selection}

Hits induced by cosmic rays can enter the ROI and contaminate track reconstruction. To select a pure laser track, crossing cosmic muons and poorly- or mis-reconstructed laser tracks should be removed. Three selection criteria are applied to the reconstructed tracks as illustrated in figure 6 .

The first two selection cuts are specifically used for eliminating cosmic tracks. In the first, tracks only pass if one of the reconstructed track-ends is close to the true laser start. The permitted differences in $X, Y$, and $Z$ are $20 \mathrm{~cm}$. No constraint is applied to the true laser end point, as unresponsive wires or cosmic muons may cause difficulties reconstructing a full laser track.

The second selection cut compares the angle of the track to the true laser track. Tracks have 3D space points defined by the wire pitch of $3 \mathrm{~mm}$. For example, a $5 \mathrm{~m}$-long track has about 1600 space points. To account for curvature along the track, the first and last 100 space points are used to fit two straight lines. The angles of the lines from each end of the track are then compared to the true laser track angle. The track is selected if both angles are less than $40^{\circ}$ from the true track angle.

The final selection step further removes the cosmic tracks and provides a quality control for the reconstructed laser tracks. Particles from cosmic rays may decay into other particles and the corresponding ionization electrons may be reconstructed as a single track. The smoothness cut would remove a cosmic track that has such a signature. Given the MicroBooNE design, no drastic E-field distortion is expected in the bulk of the TPC. We remove reconstructed laser tracks with hard kinks and wiggles, caused by artifacts irrelevant to the E-field (figure 6). The large charge deposition and broad waveforms of the laser introduces uncertainties in the hit reconstruction which can eventually lead to large deformities in the reconstructed track. We sort 3D space points in the laser tracks by their ascending $Z$ coordinate. Using the true angle and the separation in $Z$ between neighboring 3D space points, expected separations in $X$ and $Y$ can be computed. A track fails to pass the selection if $X$ or $Y$ separations are more than three times larger than expected for ten or more space points. For simplicity, mis-reconstructed tracks are removed from the selection, but one could potentially recover the well reconstructed portion of the tracks in future iterations of this work.

For calibration we only use spatial information, so no requirement on reconstructed laser energy has been applied.

From a laser scan taken in the summer of 2016 over a few hours, there are 911 upstream and 1204 downstream tracks after all selection cuts. These laser tracks are the input for the following calibration. Additionally, simulated tracks are used to assess the methodology and to estimate the systematic uncertainty.

\section{Laser track simulations}

Two simulations are used: a toy simulation evaluates the performance of the methodology with ideal tracks and a perfectly known spatial distortion. A more realistic laser simulation verifies the calculation of the spatial-displacement map and the E-field map, and provides an estimate of the systematic uncertainties of the calibration maps. The toy simulation does not integrate the detector simulation and the reconstruction, while the full laser simulation does. 

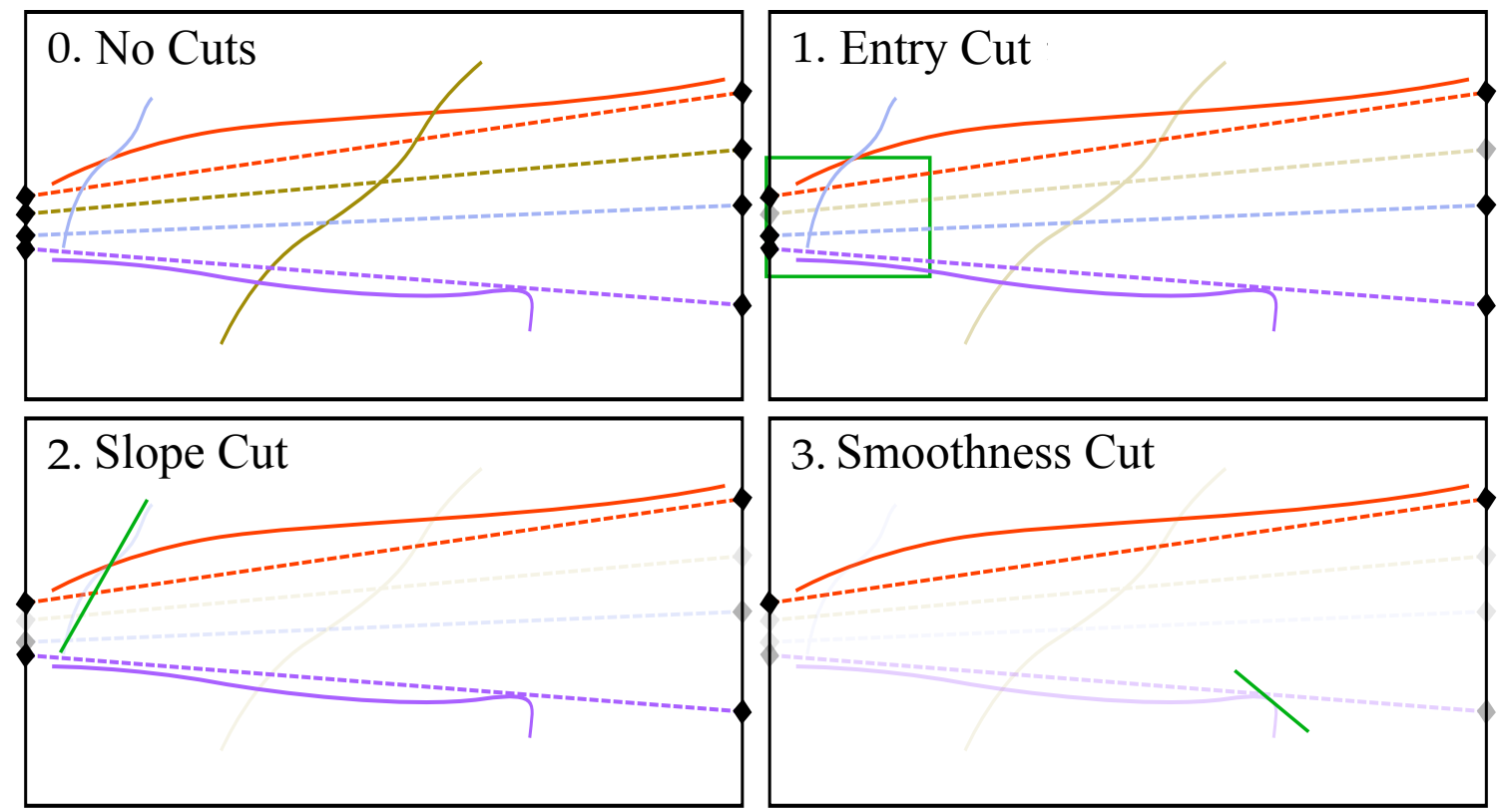

Figure 6. Illustration of the track selection steps for removal of cosmic tracks and poorly reconstructed laser tracks. This example uses laser events originating from the upstream laser sub-system. In these illustrations, the $Z$ axis is along the horizontal direction, and the vertical axis can be $X$ or $Y$. The black points on the left hand side of each TPC projection are the laser entry points in the events, and the ones on the right side are the laser exit points. The dashed lines connecting these points are the true laser beam positions. The solid lines with the matching colors represent the possible reconstructed tracks in the corresponding events. The solid brown line and the solid blue line are most likely to be cosmic tracks, and the solid purple line could be either a cosmic track or a poorly reconstructed laser track. (0) Reconstructed tracks with no selection applied. (1) Tracks pass this step if one of the track ends is within a $20 \mathrm{~cm} \times 20 \mathrm{~cm} \times 20 \mathrm{~cm}$ region (green box) from the laser entry. This region is a loose prior allowing for the effect of potential E-field distortions. The brown track would fail to pass this step, because neither of its track ends in the region of the green box. (2) Tracks pass this step if the angles of the first and last hundred space points are within $40^{\circ}$ of the true laser track angle. The blue track associated to green line would fail to pass this step, due to inconsistent track angle with the true laser track angle. (4) Tracks pass this cut if no hard kinks or wiggles, due to mis-reconstruction, are present. The purple track would fail to pass this step with a kink labelled by a green line at the end of the track. Only the red track passes all cuts.

In both simulations, a "muon gun" is used to replicate laser tracks in the Geant4 [22] stage of LArSoft ( $L A r G 4)$, where a single muon is simulated with processes such as delta electron emission and multiple Coulomb scattering disabled. No cosmic ray muons are simulated.

An E-field distortion is simulated with a charge distribution of positive ions accumulating along $X$ towards the cathode. The corresponding spatial displacement is simulated parametrically. The details are described in [23]. The simulated E-field and spatial displacement are embedded in LArSoft, with spatial distortions applied to true tracks via LArSoft.

\subsection{Toy simulation}

To avoid bias from the uncertainties of the laser system and track reconstruction, we employ the toy simulation sample to test the methodology, since only track positions are used to calculate the spatial displacement, the corresponding local drift velocity, and the E-field. 
The detector simulation is not included in the toy simulation. A raw waveform is not generated, implying that signal processing and reconstruction are not needed. The true tracks are the straight lines of ionization generated along the direction of the input muon gun. Spatial distortions are directly applied to charge deposited positions on the true tracks. We refer to the distorted tracks as the "reconstructed" tracks to be comparable with laser data, although the full reconstruction chain is not part of the toy simulation. The reconstructed tracks from the toy simulation are then processed through the track selection described in section 3.2. The surviving tracks constitute the toy simulation sample (referred as the toy simulation in the following text) for the methodology evaluation.

\subsection{Full laser simulation}

The full laser simulation is introduced to mimic the reconstructed laser data, so as to verify the complete chain of the E-field calculation using the laser system.

Unlike the toy simulation, we implement the detector simulation which includes signal processing and reconstruction in the full laser simulation. In the MicroBooNE setup, laser beams have a diameter of about $3 \mathrm{~mm}$, and they effectively ionize more than simulated muons with the default muon gun settings. Thus, a typical laser signal (raw waveform from wires) is larger than a simulated muon signal in both voltage and time spread. Therefore, the longitudinal diffusion of drifting electrons in the full laser simulation is increased to be comparable with the laser beam diameter. The ionization yield in the full laser simulation is scaled up by a factor of 2 to compensate for the greater ionization of laser beams. The scaling factors of the longitudinal diffusion and the ionization yield are chosen to allow the simulated signal to match typical laser track data, with a peak voltage of $U_{\max } \approx 80 \mathrm{mV}$ and time spread of $t_{s} \approx 10 \mu \mathrm{s}$.

The simulated laser signals are processed through the reconstruction and the track selection criteria, as described in section 3. The true tracks are the straight lines in the initial direction of the muon gun and the results of their reconstruction are the reconstructed simulated tracks. They have the same detector and reconstruction effects as the laser tracks in data. This provides comprehensive track information, which allows us to estimate the systematic uncertainties.

\section{Spatial displacement map}

In a distorted E-field, the positions of reconstructed charges in the TPC differ from their true positions. True positions are actual positions of ionization in real and simulated cases. For laser tracks, true positions are along the straight trajectory of a laser beam. Reconstructed positions are the positions calculated after the readout of wire number and timing, and will be affected by possible E-field distortions. Spatial displacement maps are used to show the difference between the reconstructed and true positions.

There are two types of spatial displacement maps used: a distortion map and a correction map. The distortion map is based on the true spatial coordinates, in which the TPC boundary follows the known mechanical structure. It shows the expected reconstructed space points given the true space points. It can be used in the simulation of TPC events including E-field distortion. We use it to verify the method of extracting the spatial displacement by comparing the calculated spatial displacement to the simulation truth. The correction map is based on the reconstructed spatial coordinates, in which the TPC boundary may be irregular. It shows the expected true space points 
given by the reconstructed space points. It can be used to calibrate the position of reconstructed TPC objects in a distorted E-field and it is used to derive the local E-field.

For an irregular E-field, it is not trivial to convert from the distortion map to the correction map. However, both maps contain related information and have many similarities.

For simplicity, the displacement maps are arranged as a regular grid. Therefore, any reconstructed position within the TPC can be translated from or to the corresponding true position by interpolation between points on the displacement grid. We set 26 bins in $X, 26$ bins in $Y$, and 101 bins in $Z$, with the first and last bins centered at the TPC boundaries. For example, along the $X$ axis, the first bin center is at $X=0 \mathrm{~cm}$, and the last bin center is at $X=254.8 \mathrm{~cm}$. The bin size is about $10 \mathrm{~cm} \times 10 \mathrm{~cm} \times 10 \mathrm{~cm}$.

\subsection{Spatial displacement vectors}

Spatial displacement vectors represent the difference between the reconstructed and true positions. The reconstructed positions are deduced from the wire waveforms, with the assumption that electrons drift in a nominal, and uniform, E-field. In practice, the E-field in MicroBooNE is distorted. The true positions are where the charged particles ionize LAr in the TPC. The distortion vectors are measured by comparing points on the true laser tracks to their corresponding reconstructed points. Alternatively, the correction vectors are measured by comparing points on the reconstructed tracks to their corresponding true points. At this stage, the distortion vectors and the correction vectors have opposite direction with the same length.

One unambiguous way of calculating the spatial displacement vectors would be to use crossing tracks. If the E-field does not change, a true position should always be reconstructed in the same position. Because E-field lines cannot cross each other, the true positions and the reconstructed positions have a one-to-one correspondence. The intersection point of two true tracks, with a small tolerance allowed, must correspond to the intersection point of their associated reconstructed tracks. It is therefore straightforward to extract the spatial displacement for those intersection points. However, given the limited TPC coverage and total numbers of laser tracks, it is rare to have crossing tracks, and this method cannot be used.

Without crossing tracks, the difficulty in determining the displacement vectors comes from the lack of a point-to-point correspondence. Even though the true laser track positions are known exactly, it is not straightforward to establish where an associated reconstructed laser track should be. This subsection introduces an alternative method using closest-point projection and track iteration to determine the spatial displacement vectors.

\subsubsection{Closest-point projection}

The closest-point projection is a simple way to give a good initial estimation on the spatial displacement vectors. We project reconstructed track points perpendicularly to the true track in 3D; an example is shown in figure 7. The vectors from the reconstructed track points (red) to their closest point on the true track (blue) are the correction vectors. The vectors with opposite directions starting from the true track (blue) to the reconstructed track points (red) are the distortion vectors.

Calculating spatial displacement vectors by closest-point projection alone introduces a dependency on the laser beam angles. It forces the displacement vectors to be perpendicular to the 
corresponding true laser tracks. We use a track iteration method to reduce the bias from the initial laser beam angles.

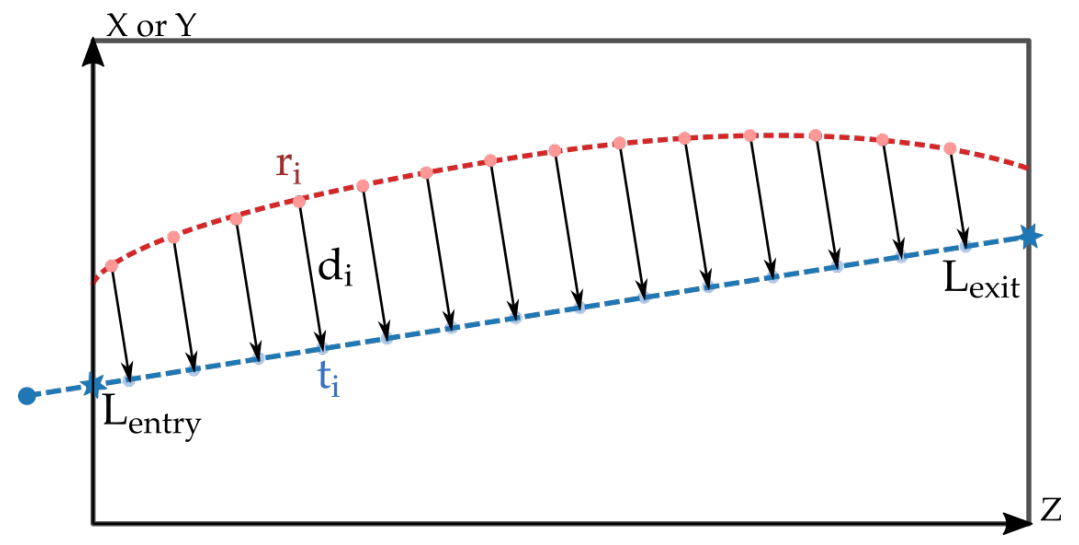

Figure 7. Diagram illustrating the closest-point projection used as the first step in calculating the spatial displacement vectors. The black box is the TPC active volume in true spatial coordinates. $Z$ is along the horizontal axis, and the vertical axis can be either $X$ or $Y$. The example shown here has a laser beam originating from the upstream sub-system, with the true laser track shown as the blue dashed line. The blue dot is the reflection point on the cold mirror which is outside of the field-cage rings. The collection of red dots is the reconstructed track. Each reconstructed point (red dot) $r_{i}$ is perpendicularly projected to the true laser track in 3D. Its corresponding point on the true track is $t_{i}$. The displacement vector $d_{i}$ starting from $r_{i}$ to $t_{i}$ is the correction vector, as shown in the diagram. The distortion vector has the same length but is in the opposite direction to the correction vector.

\subsubsection{Track iteration}

A complete description of the angular acceptance of laser tracks used in the analysis can be found in section 2.1. To maximize the spread in track angles for the correction, we split the laser tracks into two samples. Laser tracks initiating from the upstream sub-system are assembled in track sample $\mathbf{A}$, and laser tracks initiating from the downstream sub-system are assembled in track sample $\mathbf{B}$. Essentially, we use tracks from one sub-system to calculate the displacement vectors of the other in several iterative steps. Details of meshing and interpolation methods used in the following are explained in section 5.3.

We illustrate the procedure using the example of an $n$-step iteration with track samples $\mathbf{A}$ and B. Each step includes partial correction for both sample $\mathbf{A}$ and $\mathbf{B}$. At the beginning of the first step, we calculate the correction vectors of track samples $\mathbf{A}$ and $\mathbf{B}$ using closest-point projection. Then $\frac{1}{n}$ of the calculated corrections vectors of sample $\mathbf{A}$ are assigned to all the corresponding reconstructed track points. That is similarly the case for $\frac{1}{n}$ of all the calculated correction vectors of sample $\mathbf{B}$. These are referred to as the partial correction vectors of samples $\mathbf{A}$ and $\mathbf{B}$, respectively. We interpolate the partial correction vectors of track sample $\mathbf{A}$ with the reconstructed track points of sample $\mathbf{B}$ and their partial correction vectors. We refer to these interpolated partial correction vectors as the intermediate correction vectors. We then use the intermediate correction vectors to move the track points of sample A to new corrected positions. These new corrected positions as intermediate positions become pseudo reconstructed track points for the next step. We then perform the inverse procedure. The intermediate correction vectors of sample $\mathbf{B}$ are interpolated using the 
reconstructed track points of sample $\mathbf{A}$ and their partial correction vectors. Afterwards, we move the track points of sample $\mathbf{B}$ to new corrected positions. The reconstructed track points of sample A that we use for interpolation are the positions before being moved to new corrected positions. This procedure is repeated $n-1$ times. The partial correction vectors for step $i$, in the range $i=1$ to $n-1$, are a fraction $\frac{1}{n-(i-1)}$ of the correction vectors obtained by closest-point projection. After the final $n^{\text {th }}$ step, all the track points from both samples are associated to their closest point on the true tracks. This guarantees that the displacement vectors link the reconstructed track points to the corresponding points on the true tracks. The correction vectors point from the reconstructed track points to the corresponding points on the true tracks, and the distortion vectors point from the true tracks to the corresponding reconstructed track points.

By repeating the calculation with different numbers of track iteration steps, we found that three iteration steps $(n=3)$ were sufficient. The bias of the displacement maps from the simulation does not decrease noticeably with additional iteration steps.

\subsection{Boundary condition}

Independent of the E-field in the TPC, any ionization along a track at the anode position $(X=0 \mathrm{~cm})$ would be reconstructed at its actual position. Thus, in the displacement map, there is no spatial distortion at the anode.

Spatial displacements in the TPC are caused by differences between the actual E-field the electrons drift in, and the E-field used in reconstruction. The reconstructed spatial position and the true spatial position are associated with each other by the same positions at the read-out planes and the equivalent drift time. The displacement vectors connect them directly without explicit knowledge of the path of ionization electrons drifting to the read-out planes. The spatial displacement vectors are related to the E-field distortion accumulated in going from the location of the ionization to the read-out planes. Ionization electrons that are produced at the anode are immediately read out with no time to be affected by E-field distortions.

As mentioned in section 2.1, the coverage of the laser scans in MicroBooNE is restricted near the anode. The gap between the anode and the edge of the laser coverage is roughly $50 \mathrm{~cm}$. Considering the main sources of E-field distortion, space charge from cosmic rays and mechanical deformation of the detector in cold liquid Argon, we expect no drastic E-field distortion. Thus we anticipate that the changes of spatial displacements over different locations in the TPC are smooth.

We impose the boundary condition that the spatial displacement is null at the anode. Including interpolation procedures described in section 5.3, the effective region of the displacement maps increases by about $20 \%$. The validation of the displacement maps with the boundary condition included can be found in section 5.5.

\subsection{Interpolation}

In order to obtain the spatial displacement vectors on the regular grid, we interpolate them from the ones associated with the track points. The regular grid points are located at the center of each bin in the spatial displacement maps. Each bin has the same size of approximately $10 \mathrm{~cm} \times 10 \mathrm{~cm} \times 10 \mathrm{~cm}$.

As mentioned in section 2.1, laser beams do not reach the area blocked by the field-cage rings and the area near the anode. Given the bin size and the area of missing coverage, we expect 
the E-field distortion only causes small variations in spatial displacement along the regular grid. Interpolation provides a fair estimate of the spatial displacement in the low coverage area.

Interpolation is implemented in two steps. We first create a mesh of all the track points by using Delaunay triangulation. Then barycentric coordinates of the grid points in the corresponding Delaunay triangulation unit are computed, and they are further applied to achieve the spatial displacement vectors on the grid points.

We use CGAL $3 D$ triangulation $[24]^{1}[25]^{2}$ for meshing with Delaunay triangulation. For the correction map, the mesh base is the collection of all the reconstructed track points. For the distortion map, the mesh base is the collection of all the true track points which are the ends of the displacement vectors. Figure 8 is a diagram showing the interpolation procedure with a tetrahedron, a unit of the mesh. Given the barycentric coordinates of the four points defining the tetrahedron, $\mathbf{V}_{\mathbf{1}}$ to $\mathbf{V}_{\mathbf{4}}$, as well as their displacement vectors, the displacement vector at the grid point $\mathbf{P}$ can be calculated by interpolation.

The interpolation method is also used in the intermediate step of track iteration (section 5.1.2), and in the calculation of the drift velocity and E-field maps.

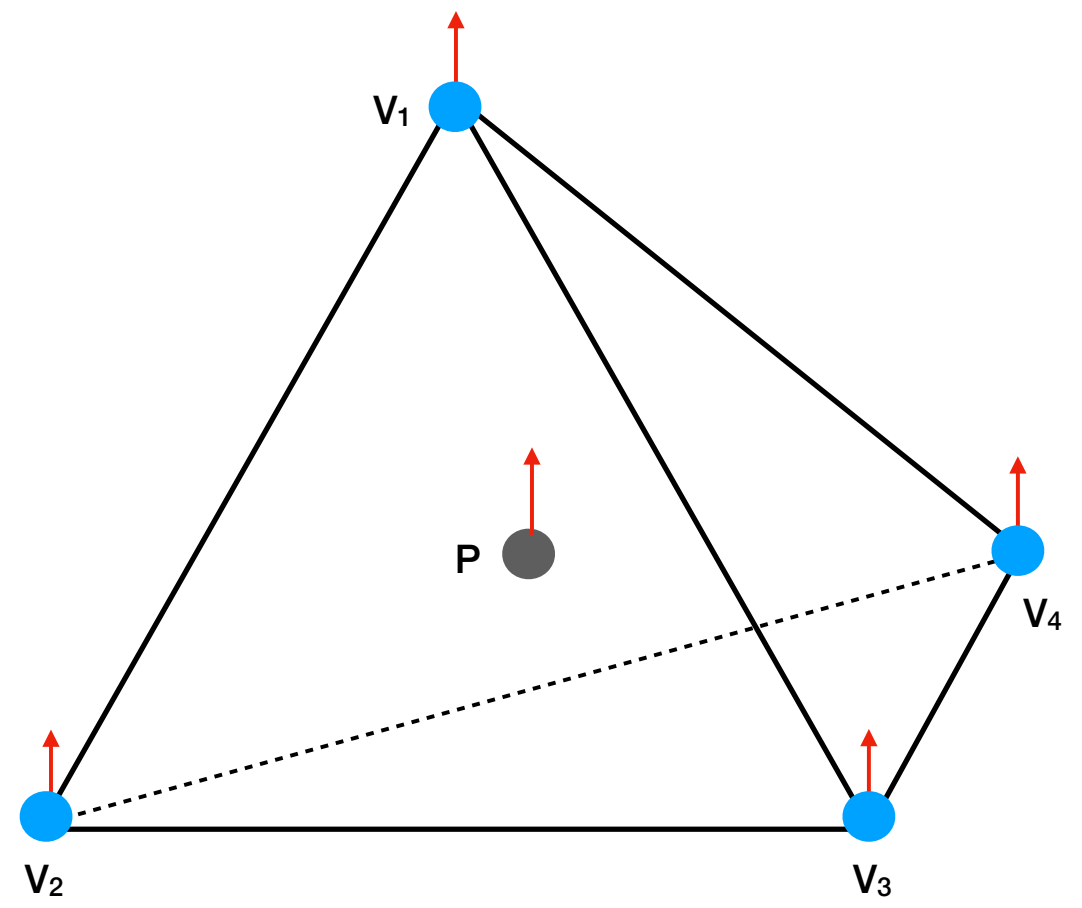

Figure 8. Diagram of a unit tetrahedron of the Delaunay Triangulation. In our application, such tetrahedrons are used as mesh units for interpolating physical quantities represented by vectors. The blue mesh points are the start of the mesh vectors and correspond to the points on the true tracks. The red arrows represent the distortion vectors. We interpolate to the regular grid point $\mathbf{P}$ (gray).

${ }^{1}$ https://doc.cgal.org/4.13/Manual/packages.html\#PkgTDS3Summary.

${ }^{2}$ https://doc.cgal.org/4.13/Manual/packages.html\#PkgTriangulation3Summary. 


\subsection{Sub-map merging}

With the interpolation procedure explained in section 5.3, the displacement vector on a grid point only depends on the tetrahedron which encloses the grid point. This means that only four track points and their displacement vectors determine the representative displacement vector in a $10 \mathrm{~cm} \times 10 \mathrm{~cm} \times 10 \mathrm{~cm}$ bin. Without subset division, the typical spacing between track points is $\sim 3 \mathrm{~mm}$ for reconstructed tracks. Hence, in a large area, the track points density is high and most of them do not contribute to the displacement map.

To make use of more of the reconstructed track points, we divide the track points of each of the original $k$ tracks into $m$ subsets. Each subset consists of points from all the tracks of both laser sub-systems, which are picked such that they are spread over the TPC volume, and will be used to create a sub-map. All sub-maps are then averaged to obtain the overall spatial displacement maps for the drift volume. To obtain a subset, from each original reconstructed track we form sub-tracks made up of the $i^{t h},(m+i)^{t h},(2 m+i)^{t h}, \ldots$ track points, for $i=1$ to $m$. In this way the track points of a sub-track are approximately equally-spaced along almost the whole length of the original track. Each sub-track shares the same true laser track as the original reconstructed track. The $k$ sub-tracks of step i then form the $i^{\text {th }}$ subset.

The track set division is performed before the calculation of displacement vectors. As the upstream laser sub-system sample $\mathbf{A}$ is separated from the downstream laser sub-system sample $\mathbf{B}$, $m$ pairs of track subsets $\left(\mathbf{A}_{1}, \mathbf{B}_{1}\right),\left(\mathbf{A}_{2}, \mathbf{B}_{2}\right), \ldots\left(\mathbf{A}_{i}, \mathbf{B}_{i}\right), \ldots\left(\mathbf{A}_{m}, \mathbf{B}_{m}\right)$ are produced, where $\mathbf{A}_{i}\left(\mathbf{B}_{i}\right)$ is the $i^{\text {th }}$ subset of sample $\mathbf{A}(\mathbf{B})$, etc. Track iteration (section 5.1.2) is implemented to calculate the displacement vectors using pairs of subsets.

Each pair of subsets produces a new mesh which leads to a different interpolation result in each bin. Thus, each pair of subsets generates a distortion sub-map and a correction sub-map, which have similar coverage as those from the original track set. The final displacement maps are calculated by averaging the displacement vectors of the $m$ sub-maps bin-by-bin. Therefore, the representative displacement vector in the same $10 \mathrm{~cm} \times 10 \mathrm{~cm} \times 10 \mathrm{~cm}$ bin is determined by many more track points and their displacement vectors.

Considering the density of the track points, we divide the original track set into 50 subsets for both the simulated track set and the laser data track set. The number of subsets is chosen to be $50(m=50)$, so that the statistics in each bin are sufficient, while maintaining the shape and characteristics of the typical unit tetrahedron. The typical spacing of track points within a track is about $3 \mathrm{~mm}$. Dividing the track set to 50 subsets makes the typical spacing of track points within a sub-track to be around $15 \mathrm{~cm}$. This is comparable with the bin size. We then average the corresponding 50 sub-maps to calculate a final map. The displacement vector on a grid point of the final map is the mean of 50 values calculated at the same point from sub-maps. Therefore, the calculation of the displacement vector on a grid point is based on $\sim 200$ track points and their displacement vectors. The averaging makes it unlikely that some individual track points with large bias could negatively affect the result on a grid point.

Due to small differences in the interpolation meshes of 50 subsets, the region with valid interpolated displacement may vary by a small amount at the edges. The standard deviation of the $50 X, Y$, and $Z$ components of the displacement vectors are taken as the statistical uncertainty of the final map. 


\subsection{Bias study}

The simulation generates straight laser tracks. Positions along these generated trajectories are in a straight line. The simulation also includes a model of the effect of space charge, and these original straight line generated space points are assigned positions shifted according to the space charge model built into the simulation. The difference between these two is referred to as the simulated displacement vector with components $d X_{\text {sim }}, d Y_{\text {sim }}$ and $d Z_{\text {sim }}$, and a magnitude $d_{\text {sim }}$. The simulated displacement vector may be obtained either with the toy or with the full laser simulation. The shifted space points in simulation undergo reconstruction as for real laser data. The resulting calculated points, when compared to the generated original straight line space points, yield a different calculated displacement vector with components $d X_{\text {calc }}, d Y_{\text {calc }}$ and $d Z_{\text {calc }}$, and a magnitude $d_{\text {calc }}$. The difference between these two displacement vectors is referred to as the bias.

The bias of the displacement maps from full laser simulation is taken as the systematic uncertainty for the displacement maps determined with data. The fact that the biases of the displacement maps from toy simulation are small (see section 5.5.1) demonstrates the effectiveness of the methodology.

We use the distortion map as an example to explain the bias study. As stated previously, the distortion map and the corresponding correction map describe similar spatial displacements which can be converted to each other. For both the distortion map and the correction map, we use the same TPC boundary as described in section 2.1, considering that the reconstruction is focused on a region of nominal active volume. The bins with calculated displacement show the rough coverage of the laser, which might be slightly smaller than the actual coverage due to the bin size. If the distortions of the E-field lead to the ionization electrons appearing to be out of the TPC active volume, the displacement vector in the edge bins of both the distortion map and the correction map may be difficult to represent. In that case, the correction map shows a smaller area than the actual TPC active volume.

\subsubsection{Bias study using toy simulation}

Figure 9 is the calculated distortion map from the toy simulation at $Z=518 \mathrm{~cm}$ (central $Z$ ), for the components $d X_{\text {calc }}=X_{\text {reco }}-X_{\text {true }}, d Y_{\text {calc }}=Y_{\text {reco }}-Y_{\text {true }}, d Z_{\text {calc }}=Z_{\text {reco }}-Z_{\text {true }}$, which are the result of the displacement calculation, applying the boundary condition and interpolation methods from section 5.1, 5.2 and 5.3. At central $Z, d X_{\text {calc }}$ has a maximum distortion at $X \approx 170 \mathrm{~cm}$ of about $\sim 4 \mathrm{~cm}$. The maximum distortion $d X_{\text {calc }}$ at the central $Z$ is in the cathode direction because of space charge, which is the main contribution to the E-field distortion in MicroBooNE, drifting towards and accumulating preferentially near the cathode. At the region closest to the anode and the cathode, $d X_{\text {calc }}$ is negligible. The distortion $d Y_{\text {calc }}$ close to the cathode has a value up to $\sim 15 \mathrm{~cm}$ and points inward. $d Y_{\text {calc }}$ is almost symmetric with respect to $Y=0 . d Z_{\text {calc }}$ is generally small and uniform.

Figure 10 shows the corresponding standard deviation of the calculated distortion within each bin at central $Z$ (section 5.4). In general, the standard deviations of $d X_{\text {calc }}, d Y_{\text {calc }}, d Z_{\text {calc }}$ are found to be small. In comparison to other distortion components, the larger standard deviation in $d Z_{\text {calc }}$ implies that the calculation of the displacement vectors is affected by the track angle. 

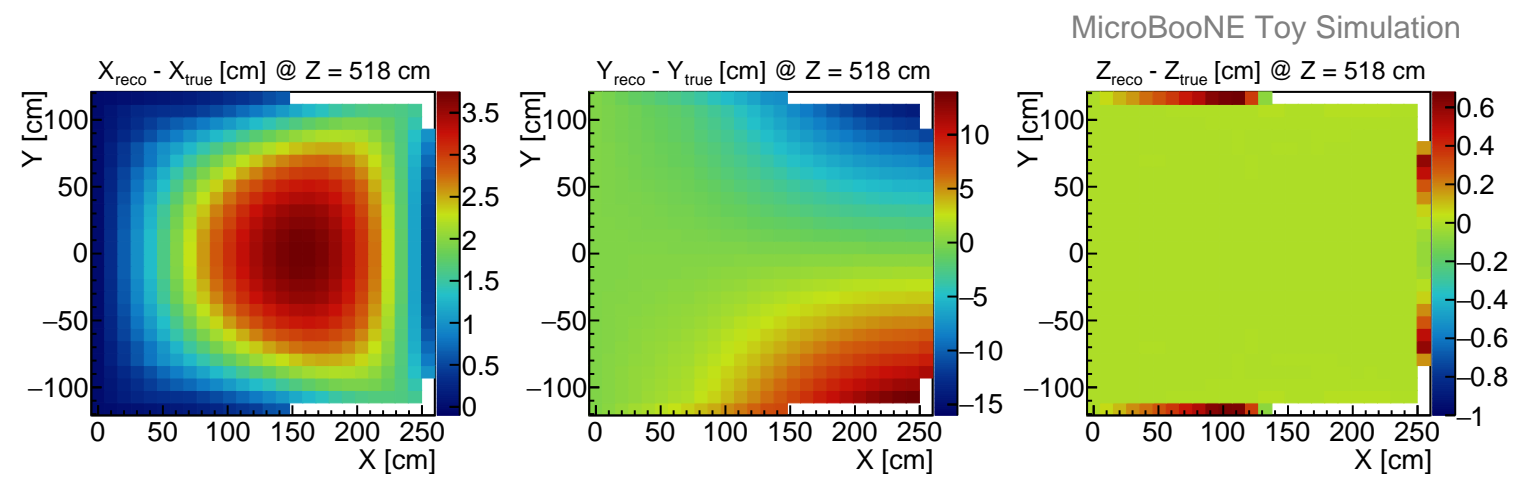

Figure 9. Calculated distortion map from a toy simulation showing a $X-Y$ slice at $Z=518 \mathrm{~cm}$ (central $Z$ ). All three components are shown: $d X_{\text {calc }}=X_{\text {reco }}-X_{\text {true }}$ (left), $d Y_{\text {calc }}=Y_{\text {reco }}-Y_{\text {true }}$ (middle), and $d Z_{\text {calc }}=Z_{\text {reco }}-Z_{\text {true }}$ (right).

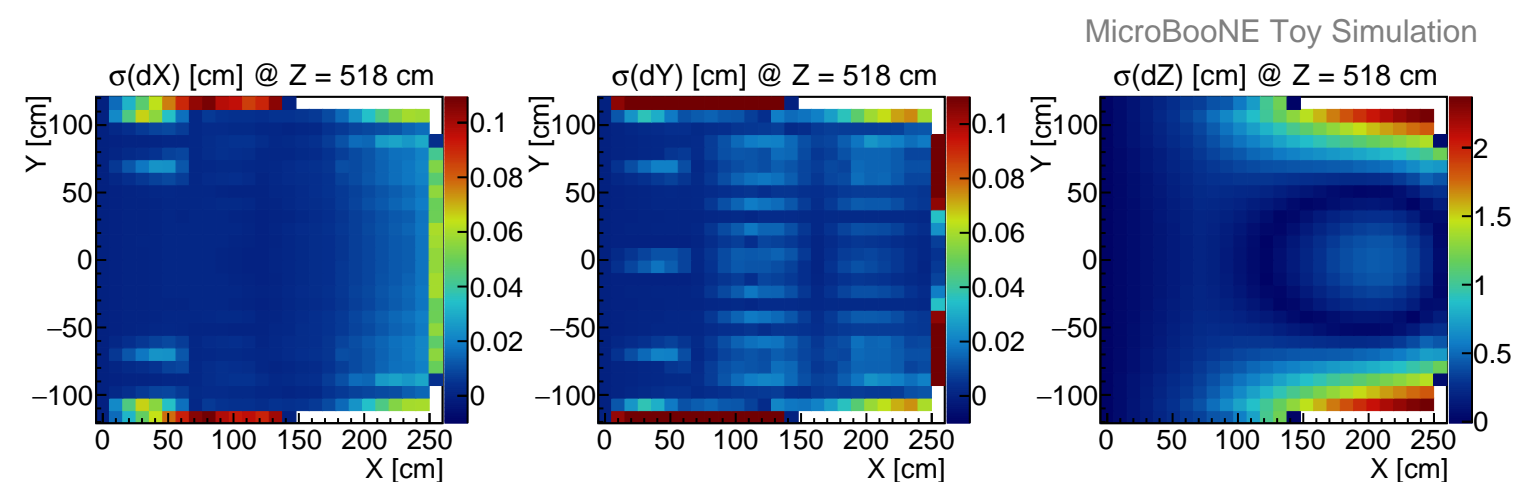

Figure 10. Standard deviation of the calculated distortion for $d X_{\text {calc }}, d Y_{\text {calc }}$ and $d Z_{\text {calc }}$ at $Z=518 \mathrm{~cm}$ (central $Z$ ) in the toy simulation.

The difference between the simulation truth and the calculated distortion is taken as the bias of the algorithm, excluding the reconstruction process. The bias $\Delta d X=d X_{\text {sim }}-d X_{\text {calc }}$, $\Delta d Y=d Y_{\text {sim }}-d Y_{\text {calc }}, \Delta d Z=d Z_{\text {sim }}-d Z_{\text {calc }}$ at central $Z$ is displayed in figure 11 . We observe good agreement, with only a small bias. The poor performance at the TPC edges is due to a combination of a lack of track coverage and effects of interpolation.

\subsubsection{Bias study using full laser simulation}

The calculated distortion map determined by the full laser simulation at the central $Z$ slice is presented in $d X_{\text {calc }}, d Y_{\text {calc }}$ and $d Z_{\text {calc }}$ components in figure 12. This distortion map is determined from the same E-field simulation [23], and thus input spatial distortion, as the one determined in the toy simulation study. For this central $Z$ slice, the main features of the distortion are similar to those observed with the toy simulation. Compared to figure 9 , the maximum distortions in $d X_{\text {calc }}$ and $d Y_{\text {calc }}$ have very similar magnitudes and similar positions, while the coverage near the cathode and at the bottom of the TPC are slightly different. A possible reason for the difference is the behavior in reconstruction with more diffuse charge deposition.

Figure 13 illustrates the measured standard deviations within each bin at central $Z$. Compared to the standard deviations of $d X_{\text {calc }}, d Y_{\text {calc }}, d Z_{\text {calc }}$ from the toy simulation (figure 10), the full laser 

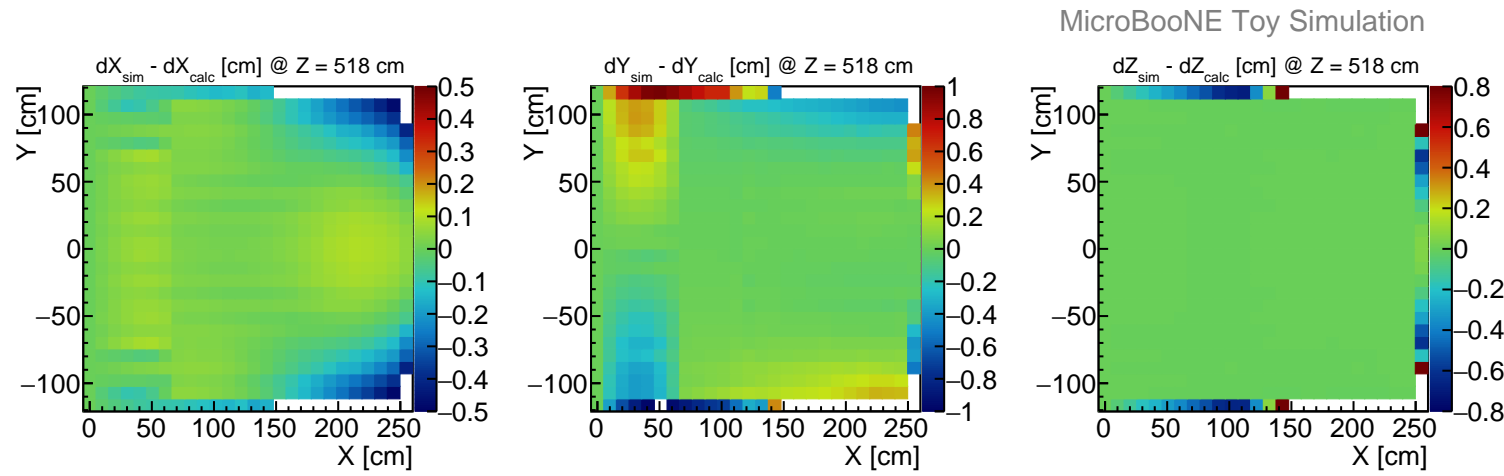

Figure 11. Bias in $\Delta d X=d X_{\text {sim }}-d X_{\text {calc }}, \Delta d Y=d Y_{\text {sim }}-d Y_{\text {calc }}, \Delta d Z=d Z_{\text {sim }}-d Z_{\text {calc }}$ from the toy simulation shown for a slice at a central slice at $Z=518 \mathrm{~cm}$. In each bin the bias is relatively small compared to the distortion shown in figure 9.

simulation standard deviations fluctuate more, but in general remain reasonably small. The larger standard deviation in $d Z_{\text {calc }}$ (at central $Z$ ) at the top and bottom of the TPC close to the cathode is at the same scale as in the toy simulation. We attribute this increase to the statistical fluctuation of distortions to the track reconstruction.

The biases $\Delta d X=d X_{\text {sim }}-d X_{\text {calc }}, \Delta d Y=d Y_{\text {sim }}-d Y_{\text {calc }}$ and $\Delta d Z=d Z_{\text {sim }}-d Z_{\text {calc }}$ at central $Z$ are shown from the full laser simulation in figure 14. In comparison with the bias from toy simulation (figure 11), the magnitude of the biases are slightly larger, but are still at the subcentimeter level. The slightly asymmetric distributions of $\Delta d Y$ and $\Delta d Z$ in figure 14 also suggest an asymmetry introduced by reconstruction. The bias, arising from the methodology, is also taken as the total systematic uncertainty of the displacement calculation, given that the estimates of other systematic uncertainties, such as of the mirror angle and the reflection point on the mirror, are all considerably smaller.
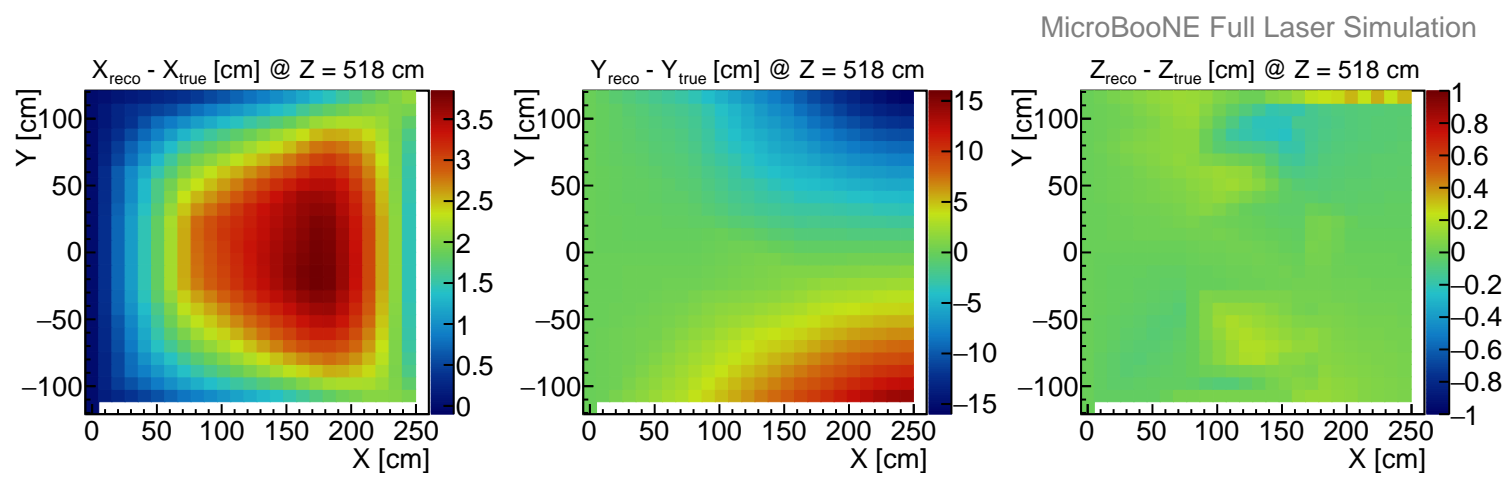

Figure 12. Calculated distortion map from the full laser simulation at a central slice at $Z=518 \mathrm{~cm}$. Shown is $d X_{\text {calc }}=X_{\text {reco }}-X_{\text {true }}$ (left), $d Y_{\text {calc }}=Y_{\text {reco }}-Y_{\text {true }}$ (middle), and $d Z_{\text {calc }}=Z_{\text {reco }}-Z_{\text {true }}$ (right).

\subsubsection{Coverage of the TPC volume with the UV-laser method}

The distortion map calculated by the full laser simulation is generally very close to the true distortion map. However, at the upstream and downstream edges of the TPC a sharp triangular edge in the 

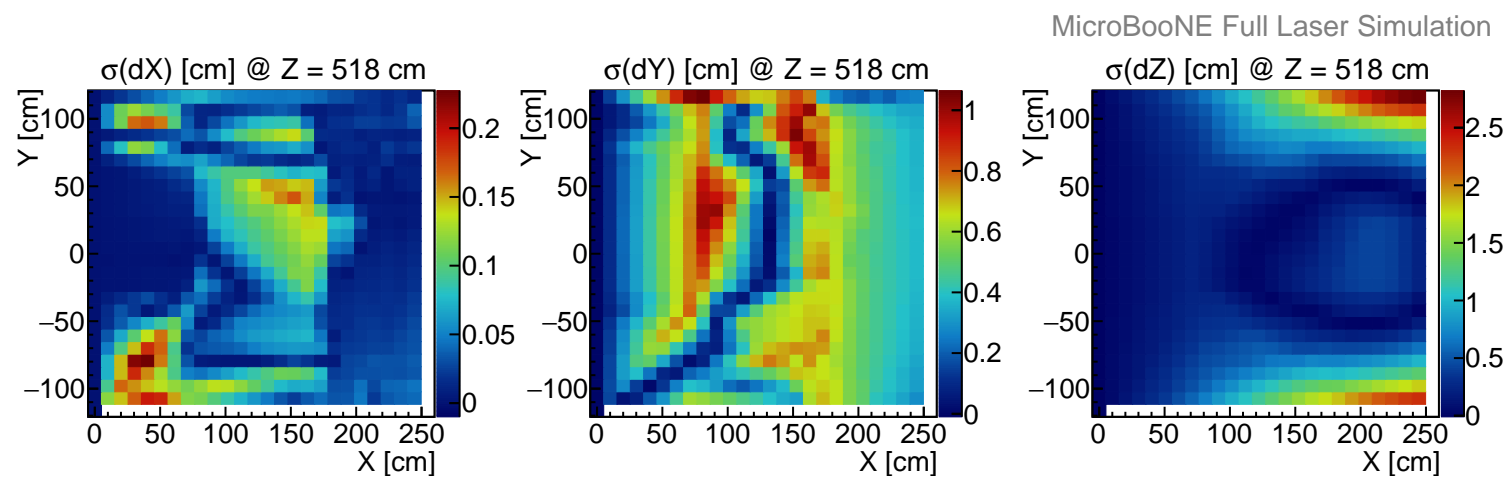

Figure 13. Standard deviation of the calculated distortion for $d X_{\text {calc }}, d Y_{\text {calc }}$, and $d Z_{\text {calc }}$ for a central slice at $Z=518 \mathrm{~cm}$ in the full laser simulation.
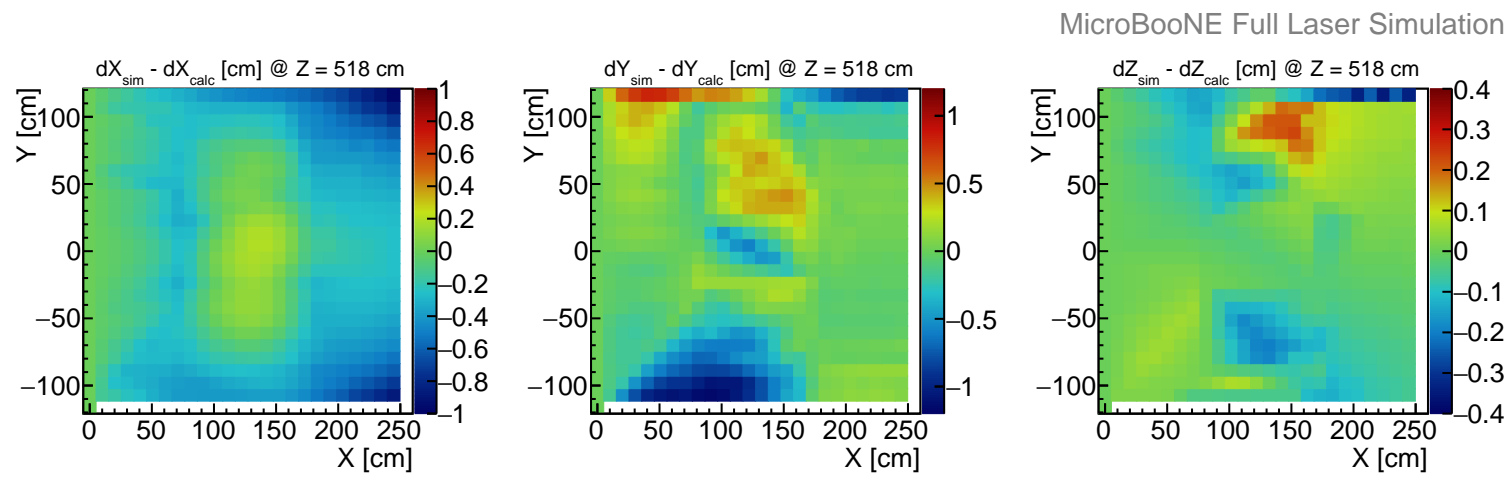

Figure 14. Bias calculated as $\Delta d X=d X_{\text {sim }}-d X_{\text {calc }}, \Delta d Y=d Y_{\text {sim }}-d Y_{\text {calc }}$, and $\Delta d Z=d Z_{\text {sim }}-d Z_{\text {calc }}$ from the full laser simulation at a central slice at $Z=518 \mathrm{~cm}$. This bias shows the overall effect of the distortion calculation, the track reconstruction, and the selection. In most of the bins, the bias is relatively small compared to the distortion.

calculated distortion from the center of the $X-Y$ to the top and bottom ends of the anode $(X=0 \mathrm{~cm})$ is noticeable in all views, as shown in figure 15. Moreover, the bias of the same upstream $Z$ slice (figure 16) shows a larger bias at the center of the $X-Y$ plane where the sharp edge is seen in figure 15. This is due to the specific placement of the laser mirrors in MicroBooNE. The bias $\Delta d Y$ are relatively large at lower $Y$ and $\Delta d Z$ reaches $\sim 10 \mathrm{~cm}$ at high $X$.

MicroBooNE being on the surface, has the copious cosmic ray flux that can also be used to determine the distortion map through a separate technique, and we are able to fill in the regions where the UV-laser method has large uncertainties. This work will be described in a future publication. We define a region of validity for the UV-laser technique as the region with small uncertainties. As these regions are at the center of the TPC and the method using cosmic ray muons could compensate for gaps at the edges of the TPC, the combination is expected to improve the overall maps.

In simulation the distortion is continuous by design; the sharp edge in the reconstructed distortion is an artificial effect caused by the algorithm. The closest-point projection technique introduces an angular dependence in the initial determination of the displacement vectors. The track iteration does reduce this bias, but is less effective at the upstream and downstream edges due to the placement of the mirrors and thus the start points of the laser tracks. Where these enter the 
TPC, they are close to collinear and point to the same spot on the mirror. In figure 3 we show that the starting points of the laser tracks are mostly located in an area not accessible by tracks from the other side. Also, tracks which reach the other end in $Z$ usually have small angle differences compared to all tracks. Imposing a boundary condition at the anode, together with interpolation, extends the local discontinuity from two points to a sharp triangle-like region in the distortion map.
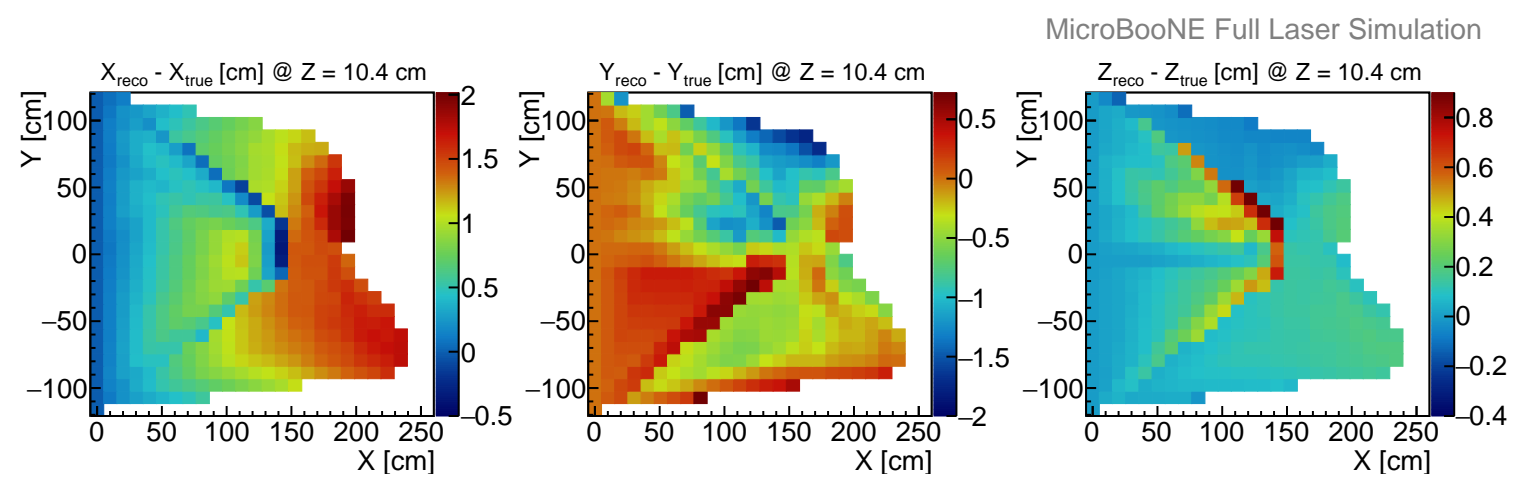

Figure 15. Calculated distortion map from the full laser simulation at an upstream slice at $Z=10.4 \mathrm{~cm}$. Shown are $d X=X_{\text {reco }}-X_{\text {true }}$ (left), $d Y=Y_{\text {reco }}-Y_{\text {true }}$ (middle), and $d Z=Z_{\text {reco }}-Z_{\text {true }}$ (right). The blank parts are where the laser does not have coverage. In this upstream slice in $Z$, a distinctive triangular pattern is noticeable from the center of the view to the top and bottom of the anode at $X=0 \mathrm{~cm}$.
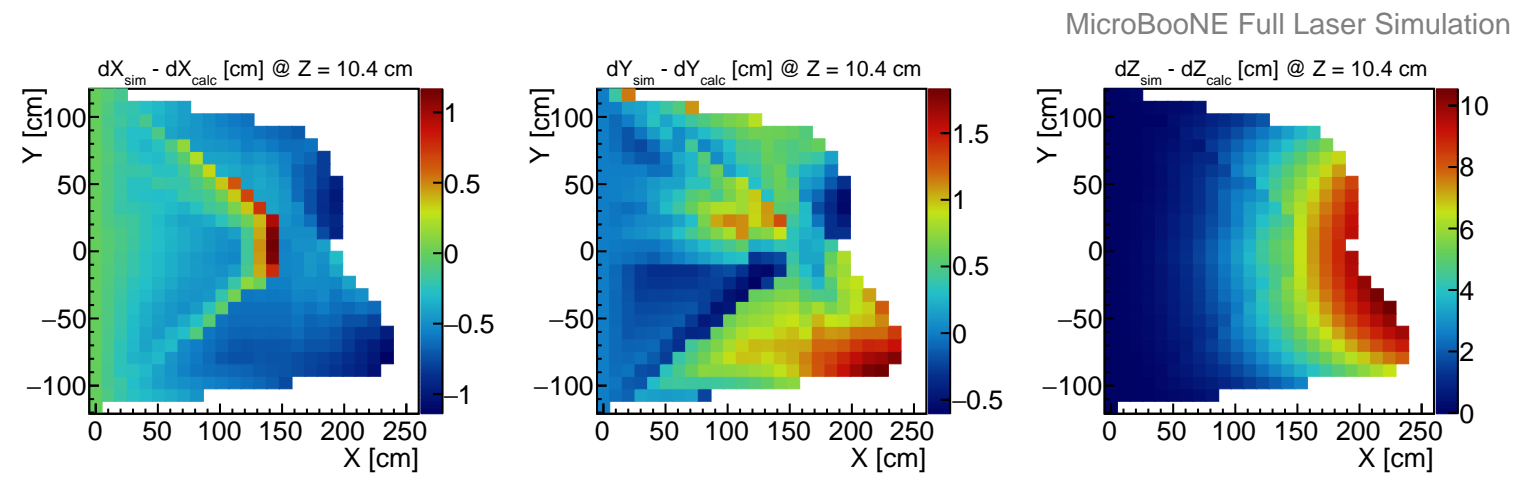

Figure 16. Bias of the calculated distortion from the full laser simulation at an upstream slice at $Z=10.4 \mathrm{~cm}$. Shown are $\Delta d X=d X_{\text {sim }}-d X_{\text {reco }}, \Delta d Y=d Y_{\text {sim }}-d Y_{\text {reco }}$ and $\Delta d Z=d Z_{\text {sim }}-d Z_{\text {reco. }}$.

Figures 17 and 18 show the distortion and the bias determined by the toy simulation in the same $Z$ slice. The sharp triangle-like region, the large bias $\Delta d Y$ close to the top and bottom near the cathode, and the large bias $\Delta d Z$ near the cathode, are very pronounced. The toy simulation is used as a reference for the full laser simulation and is not used to determine the coverage.

We proceed to define a more limited region of validity of the displacement map based on the bias and resolution maps. Figure 19 shows the bias of the calculated distortion compared to the full laser simulation truth in three components $\Delta d X=d X_{\text {sim }}-d X_{\text {calc }}, \Delta d Y=d Y_{\text {sim }}-d Y_{\text {calc }}$, and $\Delta d Z=d Z_{\text {sim }}-d Z_{\text {calc }}$, for each bin. The distribution of $\Delta d Y$ has tails up to $\pm 5 \mathrm{~cm}$, and the distribution of $\Delta d Z$ has tails up to $\pm 10 \mathrm{~cm}$. The biases $\Delta d X, \Delta d Y, \Delta d Z$ derived by toy simulation are shown in figure 20. The bias distributions are similar in figures 19 and 20. The bias distributions of 

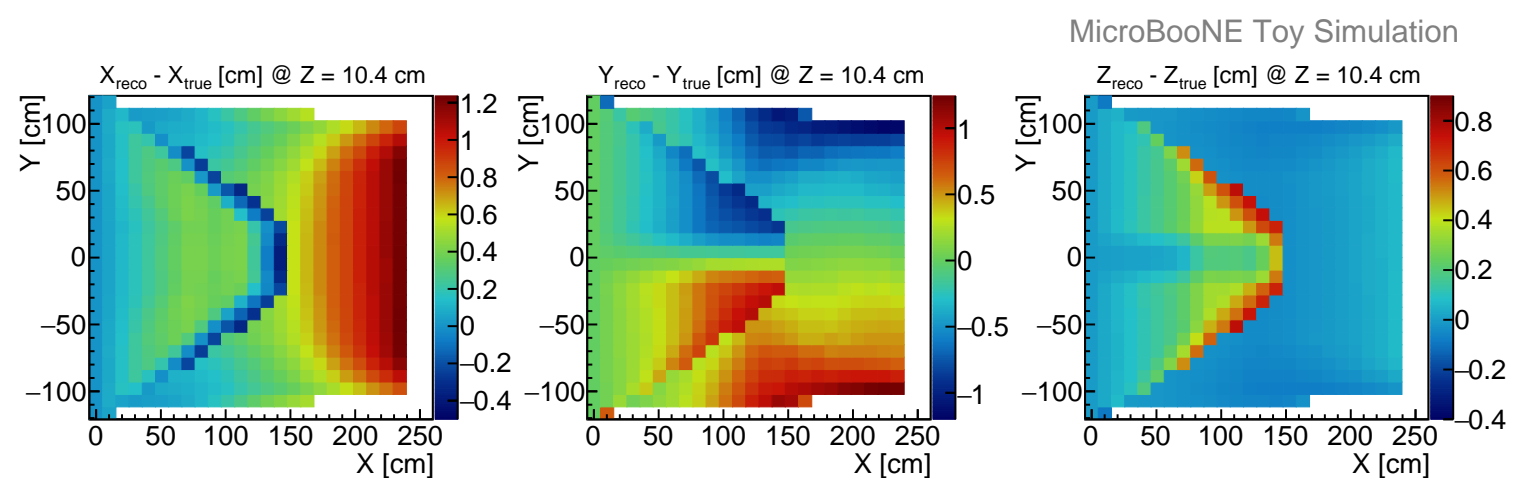

Figure 17. Calculated distortion map from the toy simulation at an upstream slice at $Z=10.4 \mathrm{~cm}$. The distinctive pattern in $Y Z$ is more pronounced and in the same region as the one from the full laser simulation.
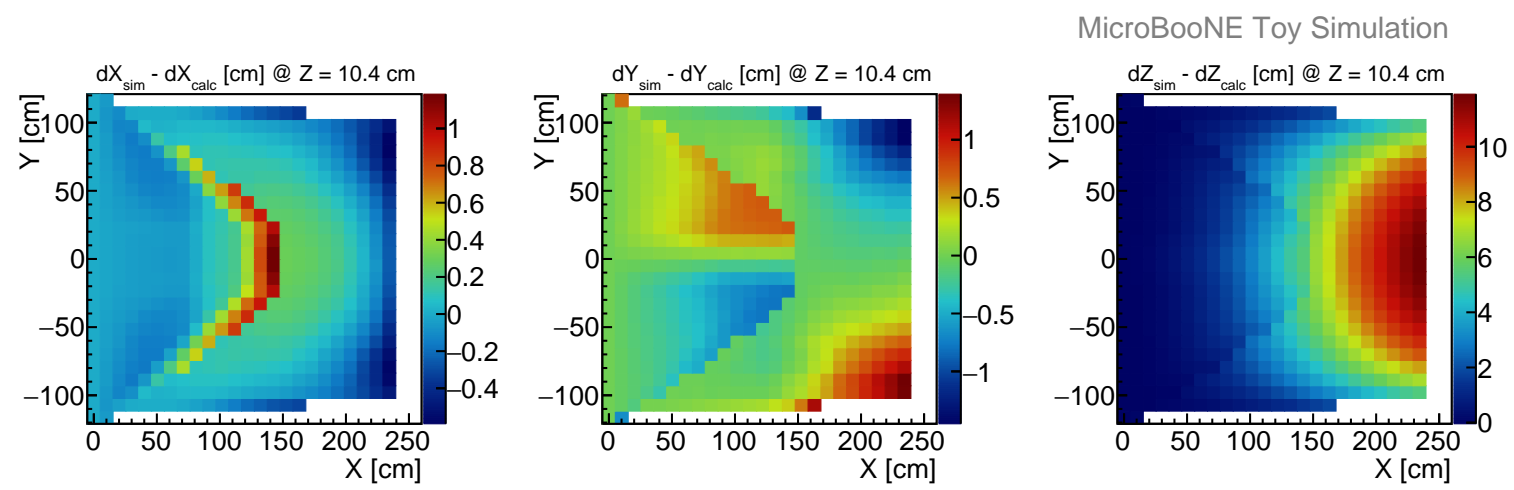

Figure 18. Bias of the calculated distortion from the toy simulation at an upstream slice at $Z=10.4 \mathrm{~cm}$, corresponding to the distortion maps shown in figure 17.

$\Delta d Y$ and $\Delta d Z$ from the full laser simulation (figures 19) are slightly asymmetric with respect to the peak at 0 . In comparison, the bias distributions from toy simulation (figures 20) are more centered around zero. The small difference is due to the additional bias introduced by the reconstruction as determined in the full laser simulation. With $d=\sqrt{d X^{2}+d Y^{2}+d Z^{2}}$ as the length of the displacement vector, the full distribution of biases $\Delta d=d_{\text {sim }}-d_{\text {calc }}$ determined by the full laser simulation over the entire TPC volume is shown in figure 21. A noticeable positive excess in $\Delta d$ is due to the calculation tending to underestimate $d_{\text {calc }}$ by the closest point projection initiated method.

Figure 22 shows the bias in $d X, d Y, d Z$ excluding the TPC edge at low $Z(0 \mathrm{~cm} \leq Z \leq 46.8 \mathrm{~cm})$ and high $Z(990 \mathrm{~cm} \leq Z \leq 1036.8 \mathrm{~cm})$. Biases in $d Y$ and $d Z$ decrease in comparison to figure 19 . They are almost symmetric and are more consistently around zero. As expected, a noticeable amount of underestimated $d$ are excluded when the valid region is limited to $46.8 \mathrm{~cm} \leq Z \leq 990 \mathrm{~cm}$. Thus, the high $\Delta d$ tail is less pronounced in figure 23 compared with the one in figure 21 .

A further restriction on $Z$ to be between $99.8 \mathrm{~cm}$ and $937 \mathrm{~cm}$ results in a narrow bias of $\Delta d X, \Delta d Y$ and $\Delta d Z$ (figure 24). The standard deviation of $\Delta d Y$ and $\Delta d Z$ are $0.40 \mathrm{~cm}$ and $0.47 \mathrm{~cm}$ respectively, which is compatible with standard deviation of $\Delta d X(0.25 \mathrm{~cm})$. The standard deviation of $\Delta d$ is also reduced to $0.37 \mathrm{~cm}$ (figure 25). The tails of these bias distributions typically end around $\pm 3 \mathrm{~cm}$. A stricter restriction to the region of validity does not significantly narrow the bias, so the final valid region is chosen to be $99.8 \mathrm{~cm} \leq Z \leq 937 \mathrm{~cm}$ and the full span of $X$ and $Y$. 

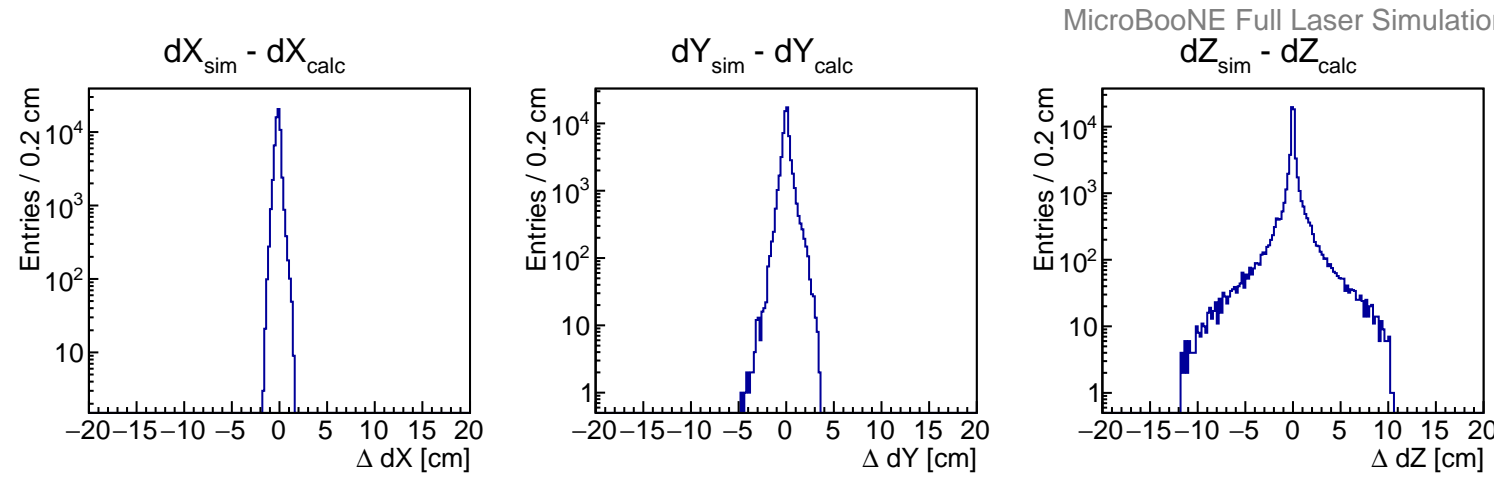

Figure 19. Bias of the calculated distortions in three components $\Delta d X, \Delta d Y$ and $\Delta d Z$ from the full laser simulation in the region with coverage. The bias distributions of $\Delta d Y$ and $\Delta d Z$ are not symmetric with respect to the peak at 0 .
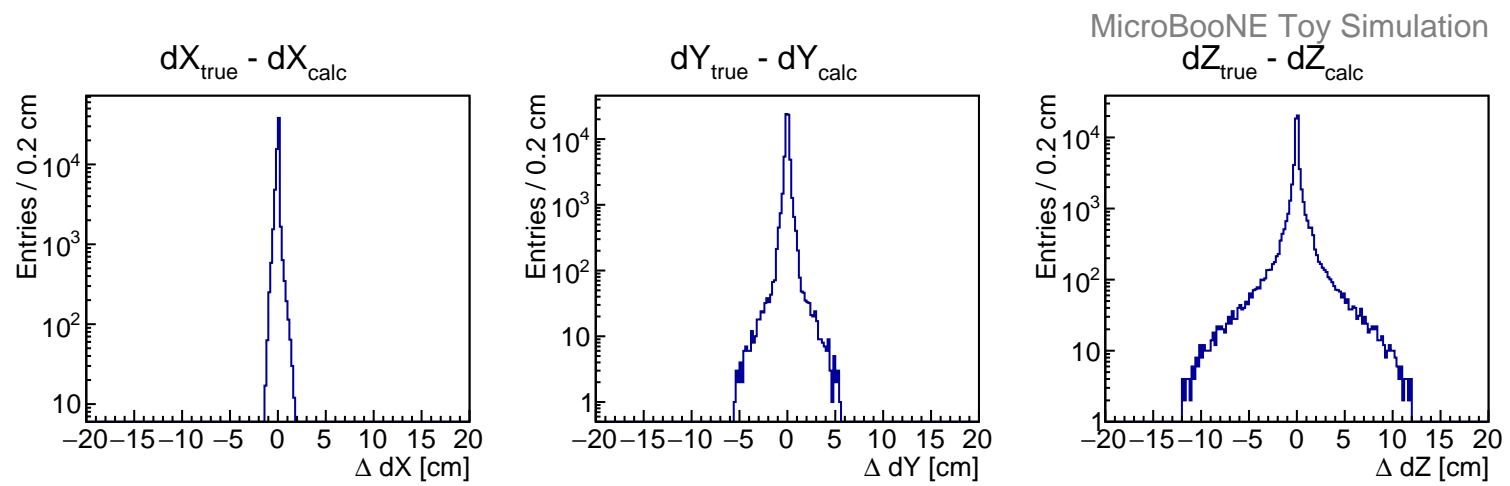

Figure 20. Bias of the calculated distortions in three components $\Delta d X, \Delta d Y$ and $\Delta d Z$ from the toy simulation in the region with coverage. The bias distributions are symmetric around the peak at 0 .

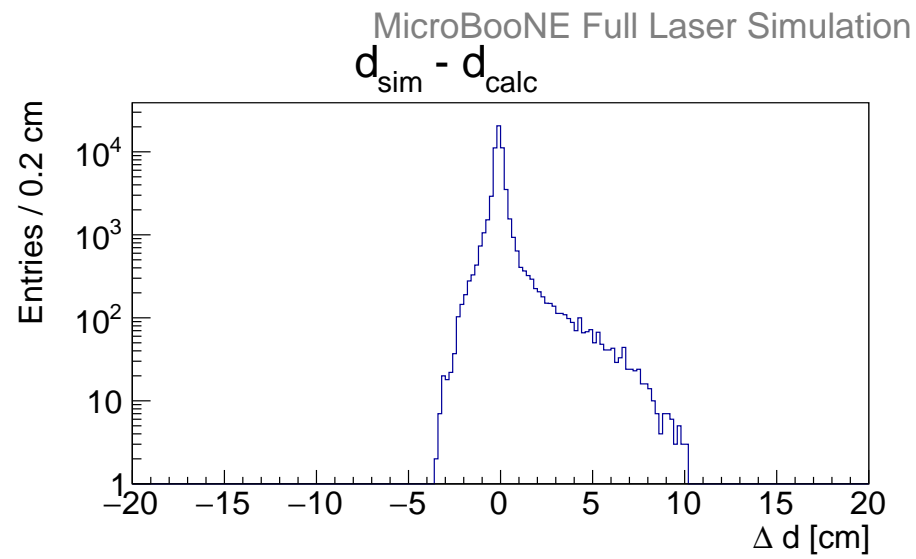

Figure 21. The magnitude $d$ of the distortion vector, where $\Delta d=d_{\text {sim }}-d_{\text {calc }}$ is the bias of the calculated distortion from the full laser simulation of the map region with laser coverage. A excessive tail is populated at $\Delta d>0$. It is expected that overall the calculation distortion is smaller than the true distortion, because of the step of closest-point projection. 

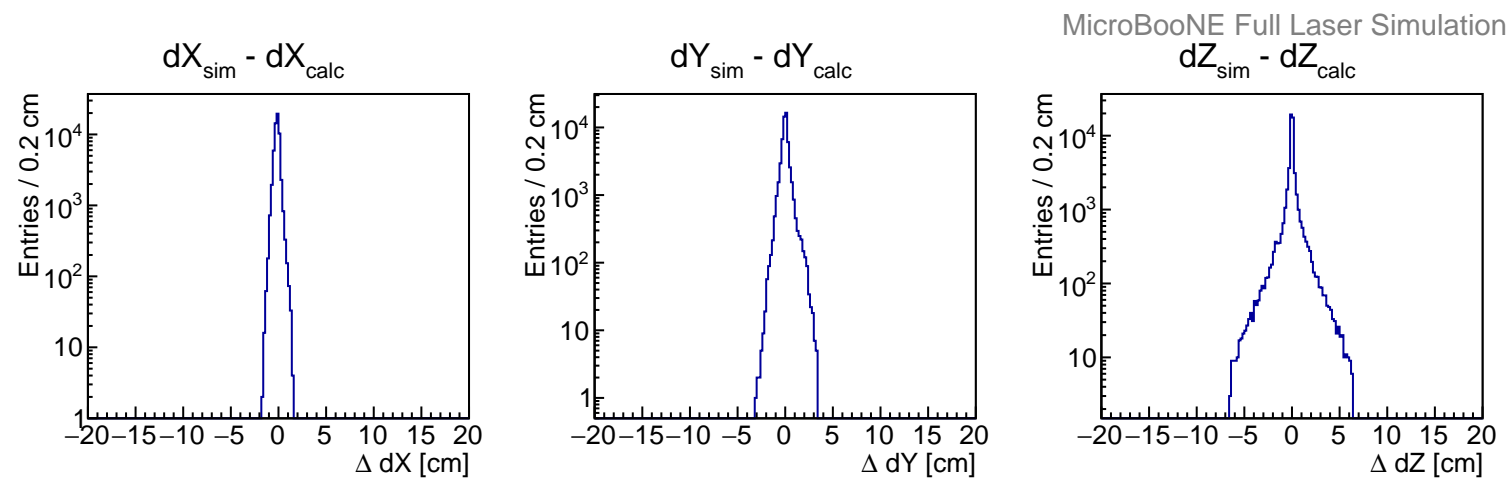

Figure 22. Bias of the calculated distortion in three components $\Delta d X, \Delta d Y$ and $\Delta d Z$ from the full laser simulation. In the region of $46.8 \mathrm{~cm} \leq Z \leq 990 \mathrm{~cm}$, the bias distributions are centered at 0 and symmetric. The spread of the bias distributions are also smaller compared to the ones including high $Z$ and low $Z$ ends.

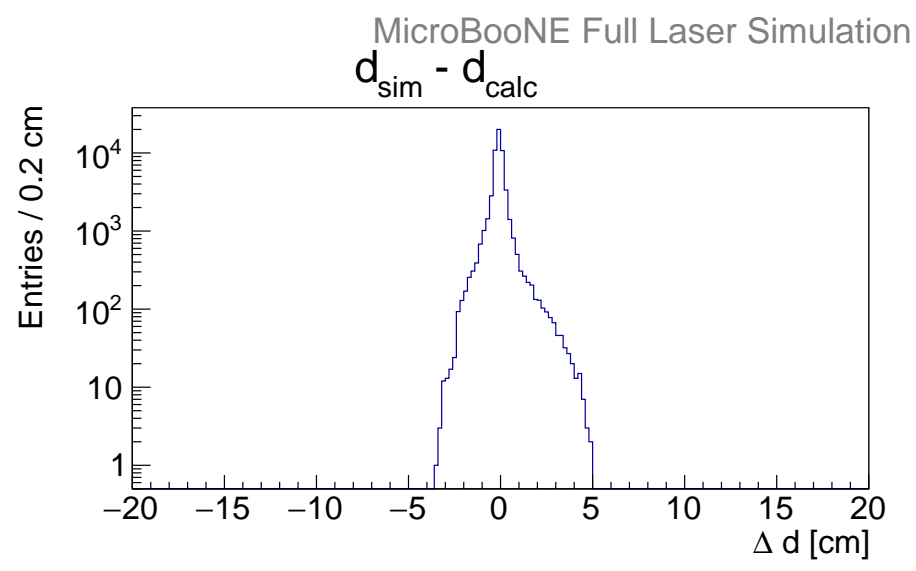

Figure 23. Magnitude $\Delta d$ of the bias of the calculated distortion from the full laser simulation. The region of $46.8 \mathrm{~cm} \leq Z \leq 990 \mathrm{~cm}$ in the TPC is included, excluding high $Z$ and low $Z$ coverage. With this selection the tail of $\Delta d>0$ is largely reduced.
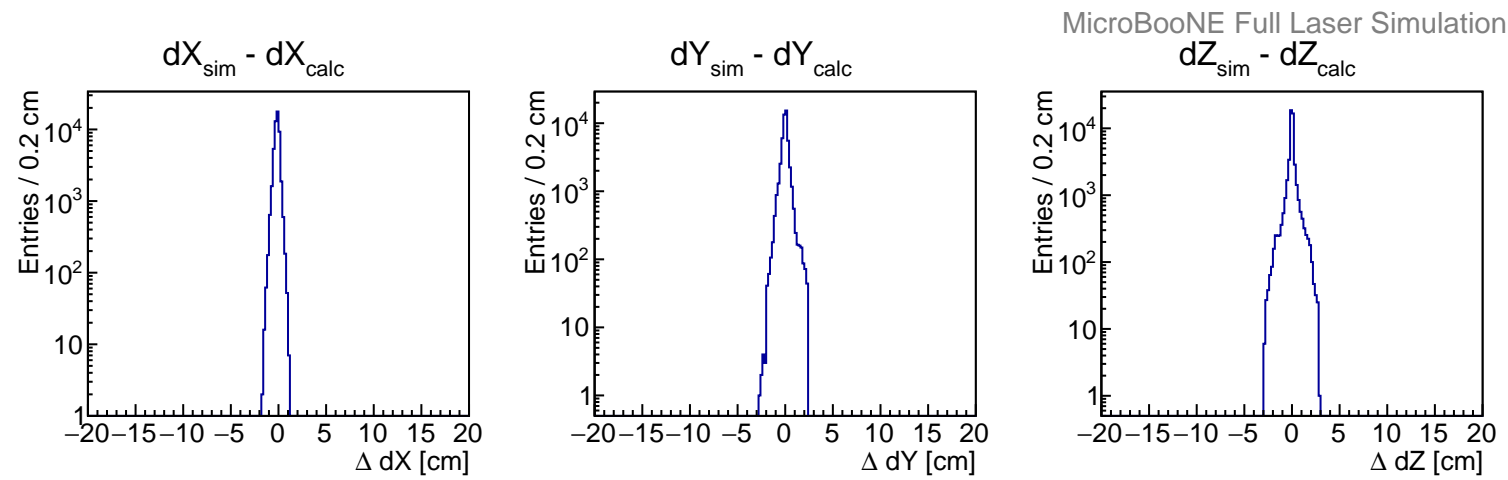

Figure 24. Bias of the calculated distortion in the three components $\Delta d X, \Delta d Y$, and $\Delta d Z$ from the full laser simulation. Only the region of $99.8 \mathrm{~cm} \leq Z \leq 937 \mathrm{~cm}$ is included. The bias distribution are now closer to symmetric around the peak at 0 . The standard deviations of each components are $\Delta d X \sim 0.2 \mathrm{~cm}$, $\Delta d Y \sim 0.4 \mathrm{~cm}$, and $\Delta d Z \sim 0.4 \mathrm{~cm}$. 


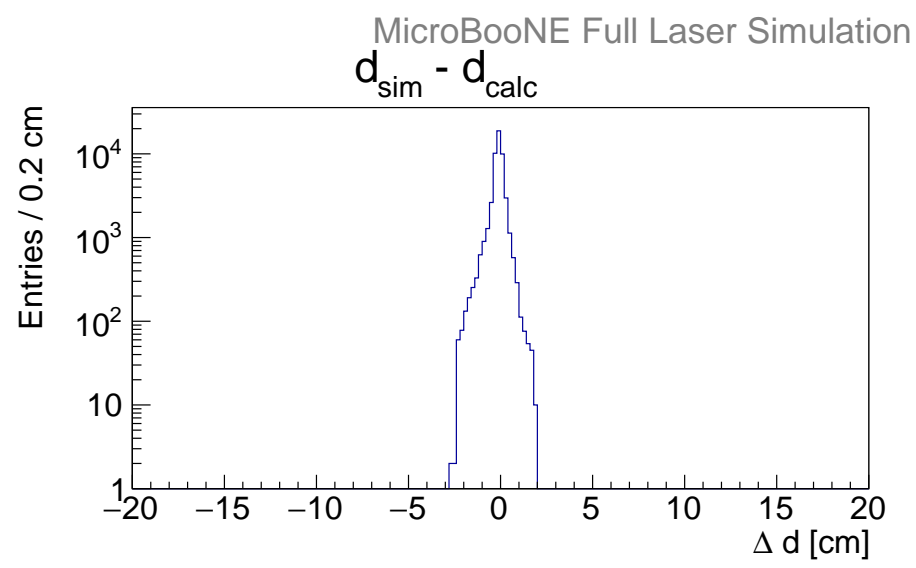

Figure 25. Magnitude $\Delta d$ of the bias of the calculated distortion from the full laser simulation, with $99.8 \mathrm{~cm} \leq Z \leq 937 \mathrm{~cm}$. $\Delta d$ of each bin in the region of validity is relatively small. The standard deviation is $\Delta d \sim 0.4 \mathrm{~cm}$.

The methodology for calculating displacement maps, which has been validated by toy and full laser simulations, can be regarded as a valid technique. Room for improvement lays in the calculation of displacement vectors and laser track reconstruction.

\section{E-field and drift velocity maps}

Although the E-field for drifting ionization electrons is designed to be uniform in between the cathode and anode planes, this is difficult to achieve in practice. Both the TPC geometry and space charge effect can distort the E-field. To better identify charged particles and their locations in the TPC, a precise mapping of the local E-field at each point is necessary.

The E-field is calculated and then assigned to the TPC active volume in true spatial coordinates on an adjustable regular grid. We choose 26 bins along $X, 26$ bins along $Y$ and 101 bins along $Z$ with the TPC edge at the center of the edge-most bins. With the interpolation procedures, the E-field can be determined at any position in the valid map area.

In the following, we describe how the E-field is extracted from distortions maps derived from UV-laser data. In principle, the technique can be also applied to any calibration data source.

\subsection{Field lines}

E-field lines extend from the anode to the cathode. Ideally, ionization electrons generated anywhere along a field line drift to the same position at the anode. Since the ionization electrons on a field line share the same read-out position, they are reconstructed along a line which passes that read-out position and is perpendicular to the anode plane.

We take the correction map, which has a regular grid in reconstructed spatial coordinates, as the input to the E-field calculation. In figure 26, the gray dots are regular grid points in reconstructed spatial coordinates. The dots along the dashed line all correspond to the same read-out position (orange). The red arrows are the correction vectors on those gray grid points, and the blue dots are the corresponding true positions. We organize the blue dots by the distance of their corresponding reconstructed grid points to their read-out position. The connection from that read-out position to 
the first blue dot, represents the field line in this step. Similarly, the continuous connection from a blue dot to the next blue dot forms the field line.

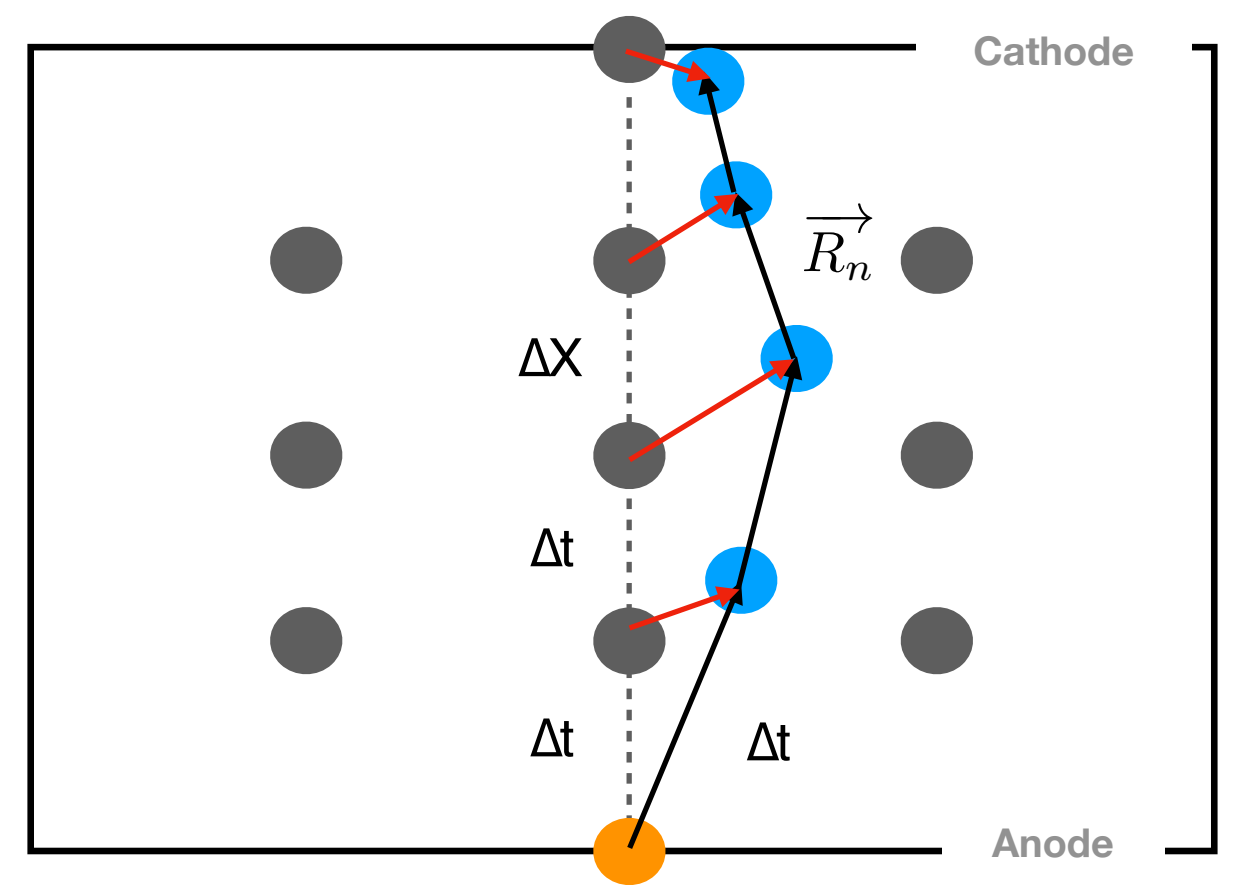

Figure 26. Diagram showing how the E-field is calculated. The gray dots are regular grid points in reconstructed spatial coordinates, separated by $\Delta x=v_{0} \Delta t$, where $\Delta t$ is the fixed time sampling of the TPC, and $v_{0}$ is the nominal drift velocity. With no field distortion, points read out at the anode separated by $\Delta t$ are placed along the dotted line perpendicular to the anode. However, with a distorted field the points' true position is given by the red correction vectors, which take us to the true blue points. The blue points are the positions of the actual energy deposition in true spatial coordinates, these are also spaced by $\Delta t$. The red arrows represent the correction vector pointing from the reconstructed to the corresponding true positions. Ionization electrons drifting from any blue dots along a field line are read out at the same orange point at the anode. Thus the line connecting these points, the spatial vector at each step $\vec{R}_{\mathrm{n}}$, follows the E-field. The black arrows indicate the direction of the derived E-field at each step.

Figure 27 shows the field lines derived from laser data at $Y=0$ (central $Y$ ) and at $Z=518 \mathrm{~cm}$ (central $Z$ ). The field lines are smooth. No kinks and no crossing lines are observed. The distortion in the direction of the E-field is pronounced at central $Z$. Close to the cathode, the E-field bends outwards. Thus, in reconstructed spatial coordinates, the corner near the top and bottom edges of the cathode is not seen. The gray boxes in figure 27 indicate the projection of the TPC active volume. In principle, the field lines should be contained within the TPC active volume. The top two field lines at central $Z$ (bottom plot) are outside the gray box by $\sim 1 \mathrm{~cm}$. The E-field exists outside the TPC active volume around the boundaries because the field-cage rings are a few centimeters away from the active (instrumented by wires) volume boundaries. The E-field near the field-cage rings is typically stronger. This effect may be physical, but the position uncertainties in this region are large, on the order of $1 \mathrm{~cm}$. Also, the field lines extend slightly beyond the gray box in the $X$ direction. We obtain $X$ coordinates via the nominal drift velocity along $X$ in the TPC. The nominal drift velocity $\left(v_{0}\right)$ is $1.098 \mathrm{~mm} \mathrm{\mu s}^{-1}$. We take the cathode position as $254.8 \mathrm{~cm}$ from an estimate of 
the TPC drift length in MicroBooNE at a LAr temperature of $(89 \mathrm{~K})$. This value has an uncertainty of about $1 \mathrm{~cm}$ along $X$.

Some field lines in figure 27 do not extend across the entire drift length due to a lack of laser coverage. The asymmetry in $Z$ (upstream and downstream) may be due to the difference in the two laser positions and pulse energy settings. While the asymmetry in $Y$ is likely due to the fact that the dichroic mirrors have decreasing reflectance efficiency for larger incident angle.

Field lines in the middle of $Y(Z-X$ view)

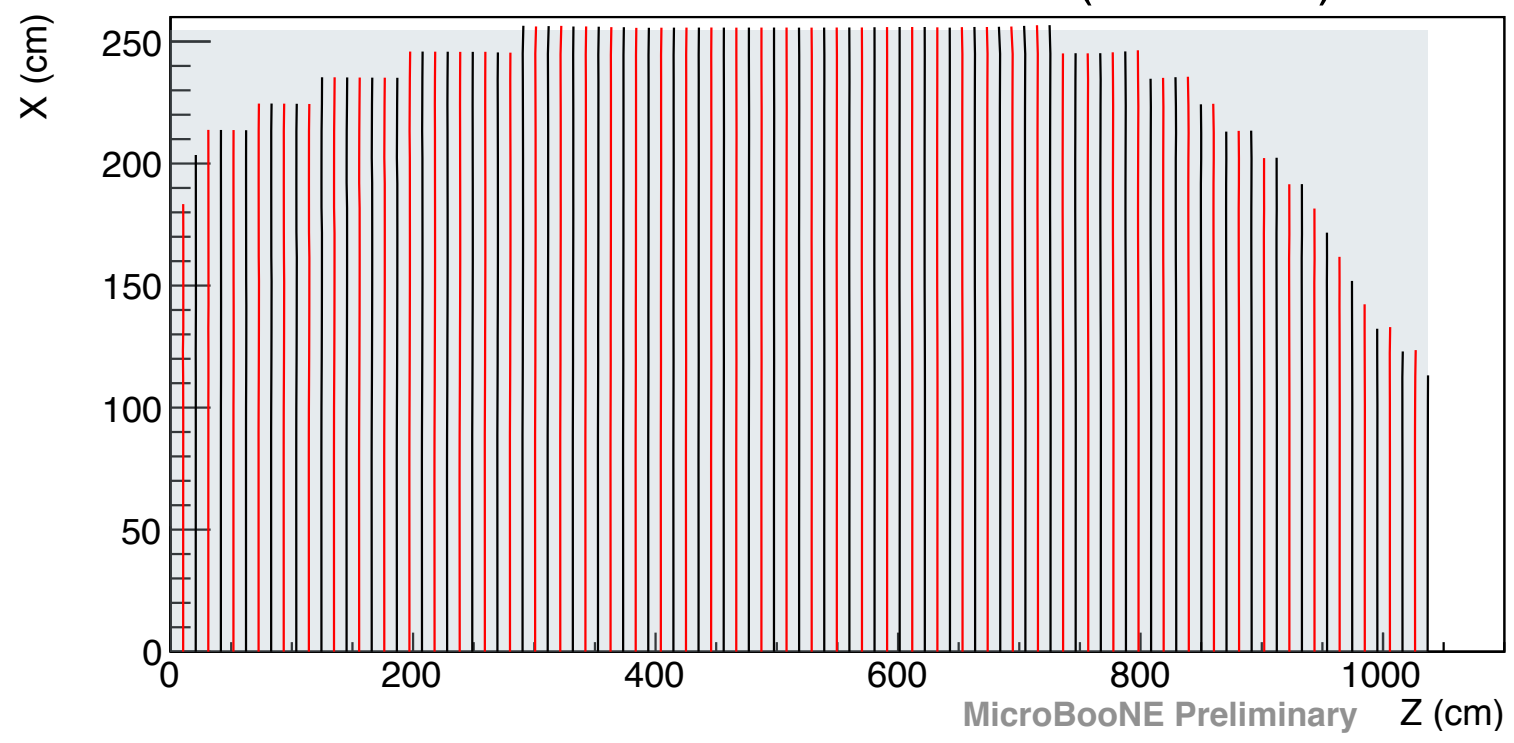

Field lines in the middle of $Z(X-Y$ view)

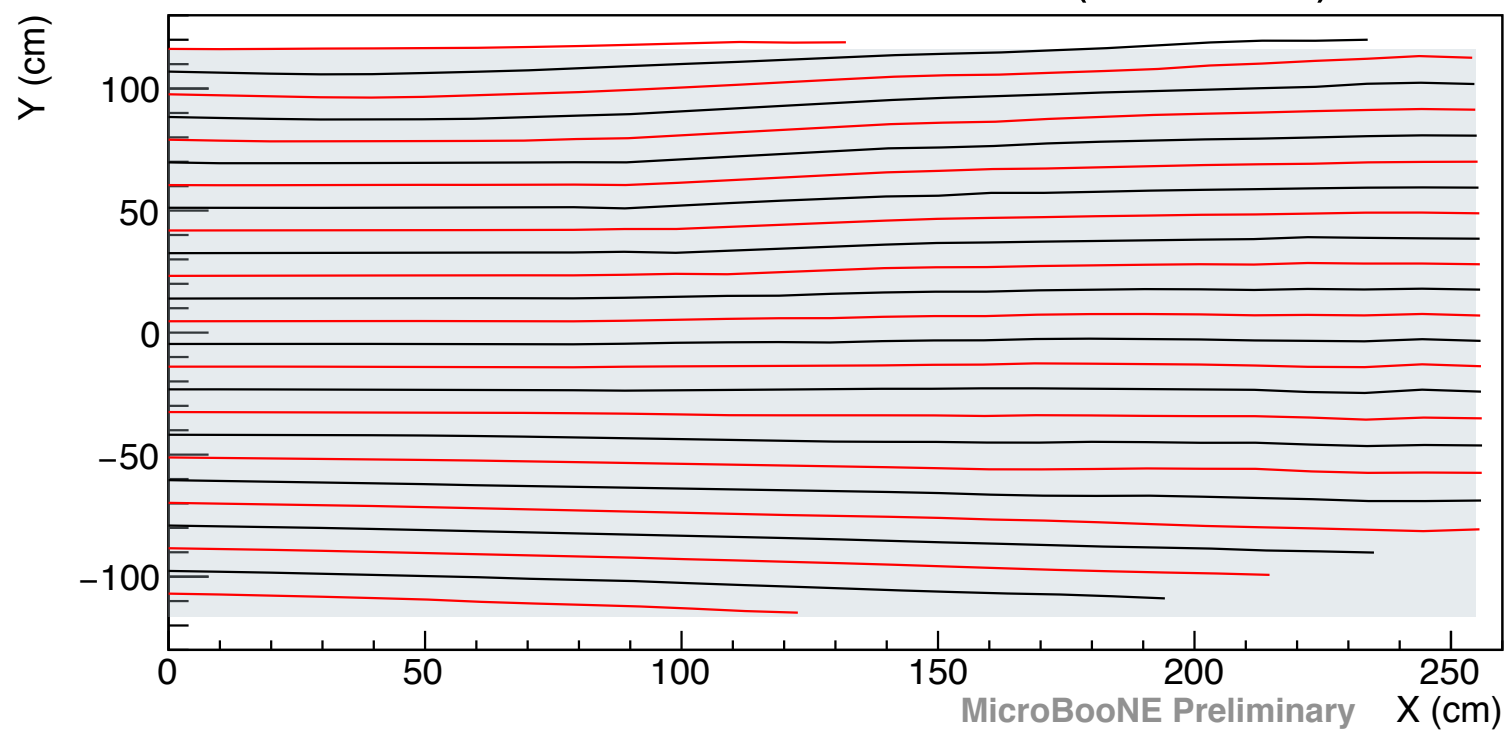

Figure 27. The upper plot shows the projection of the field lines at a central slice in $Y(Y=0)$, and the bottom plot shows the projection of field lines at central $Z(Z=518 \mathrm{~cm})$. The field lines are derived from laser data. Different line colors are used to distinguish neighboring field lines. The gray box shows the projection of the TPC active volume in true spatial coordinates. 


\subsection{Extraction of the local drift velocity}

We illustrate the calculation of the local drift velocity using figure 26. We define a step as going from the read-out position to the first true track point position, or from one track point to the next in true coordinates. Ionization electrons from the first true track point drift to the anode plane and thus the readout position in a time $\Delta t$. The reconstruction procedure, assuming a uniform E-field perpendicular to the read-out plane, assigns the position of the first point at a distance $X_{1}=\left|v_{0}\right| \cdot \Delta t$. All the reconstructed track points are spaced by $\Delta X=\left|v_{0}\right| \cdot \Delta t$ from each other, as they are on a regular grid in reconstructed spatial coordinates. The nominal drift velocity, $v_{0}$, is taken as constant along $X$, so the time difference from any gray point to the next gray point is also $\Delta t$. The $n$-th track point in reconstructed coordinates is associated to the $n$-th true point, where $n$ is an integer between 1 and 24 for the grid used here. The time which is used in the reconstruction of the $n$-th point is $n \Delta t$, so the drift time from the $n$-th point to the readout point is also $n \Delta t$. Similarly, the time associated to the $(n+1)$-th reconstructed or true points is $(n+1) \Delta t$. Therefore, the time of any step $n$ is $\Delta t$, which is the same as the time in between two neighboring gray points along $X$.

We assume the local drift velocity is constant during each step. The step vector $\vec{R}_{n}$ points from the $n$-th to the $(n+1)$-th true point. Eq. (6.1) shows the relation between $\left|\vec{R}_{n}\right|$ and $\Delta X$,

$$
\frac{\left|\vec{R}_{n}\right|}{\Delta X}=\frac{\left|\vec{v}_{n}\right| \Delta t}{\left|\vec{v}_{0}\right| \Delta t}
$$

Thus, the local drift velocity can be calculated as:

$$
\left|\vec{v}_{n}\right|=\frac{\left|\vec{R}_{n}\right|}{\Delta X}\left|\vec{v}_{0}\right|
$$

The local drift velocity $\vec{v}_{n}$ has the opposite direction to $\vec{R}_{n}$. We assign $\vec{v}_{n}$ to the center of step $n$.

If the correction vector for the $n$th reconstructed point has a large uncertainty, it only affects $\vec{R}_{n}$ and $\overrightarrow{R_{n+1}}$. Thus, only the local drift velocity $\overrightarrow{v_{n}}$ and $\overrightarrow{v_{n+1}}$ are influenced.

If there is no spatial displacement, any local drift velocity, $\vec{v}_{n}$, would be the same as the nominal drift velocity, $\vec{v}_{0}$. If the correction vectors on the reconstructed points are ideal, the magnitude of the assumed nominal drift velocity, $\left|\vec{v}_{0}\right|$, is irrelevant for the measurement of the velocity map. Any deviation would be absorbed by the correction map and the step vector $\vec{R}_{n}$ would remain unchanged. The local drift velocity, $\left|\vec{v}_{n}\right|=\frac{\left|\vec{R}_{n}\right|}{\Delta t}=\frac{\left|\vec{R}_{n}\right|}{\Delta X^{\prime}}\left|\vec{v}_{0}^{\prime}\right|=\frac{\left|\vec{R}_{n}\right|}{\Delta X}\left|\vec{v}_{0}\right|$, also would not change. However, the displacement vectors calculated as described in section 5.1 have an angular dependence on the laser tracks. Thus, the magnitude of the nominal drift velocity $\left|\vec{v}_{0}\right|$ can be relevant to the local drift velocity calculation.

The local drift velocity vectors on the regular grid are then interpolated from the drift velocities of the local steps as described in section 5.3.

\subsection{Extraction of the local E-field}

The local E-field is derived from the local drift velocity map. The direction of the E-field is the same as the step vector $\vec{R}_{n}$. The magnitude of the local E-field is determined from the corresponding local drift velocity as illustrated in figure 28 . The drift speed increases from $\sim 0.20 \mathrm{~mm} \mathrm{ss}^{-1}$ to $\sim 1.75 \mathrm{~mm} \mathrm{us}^{-1}$ when the corresponding E-field increases from $\sim 0.02 \mathrm{kV} \mathrm{cm}^{-1}$ to $\sim 0.66 \mathrm{kV} \mathrm{cm}^{-1}$. 
The drift speed as a function of E-field is taken from the LArSoft package and from refs. [26, 27]. The drift speed as a parametric function of the E-field and LAr temperature is given in ref. [27]. In MicroBooNE, the LAr temperature is stably maintained at $89 \mathrm{~K}$, which we use in the drift speed function.

The drift speed has a non-linear, monotonic dependence on the E-field. Thus one can determine the local E-field from the measured local drift speed magnitude and direction. We then use bisection to numerically determine the magnitude of the local E-field. When the distortion of the E-field is not dramatic, the change of drift speed is nearly linear around the nominal field $\left|\vec{E}_{0}\right|=0.274 \mathrm{kV} \mathrm{cm}^{-1}$.

We assign the local E-field vectors to the beginning of the steps (blue points, orange point if the given position is on the anode) shown in figure 26. They share the same positions as the local drift velocities of the steps. We also set the average E-field of two neighboring steps to the midpoint of those two steps. The start of the first step and the end of the last step use the local E-field of the first and last step. The local E-field on the regular grid is formed by interpolation as described in section 5.3.

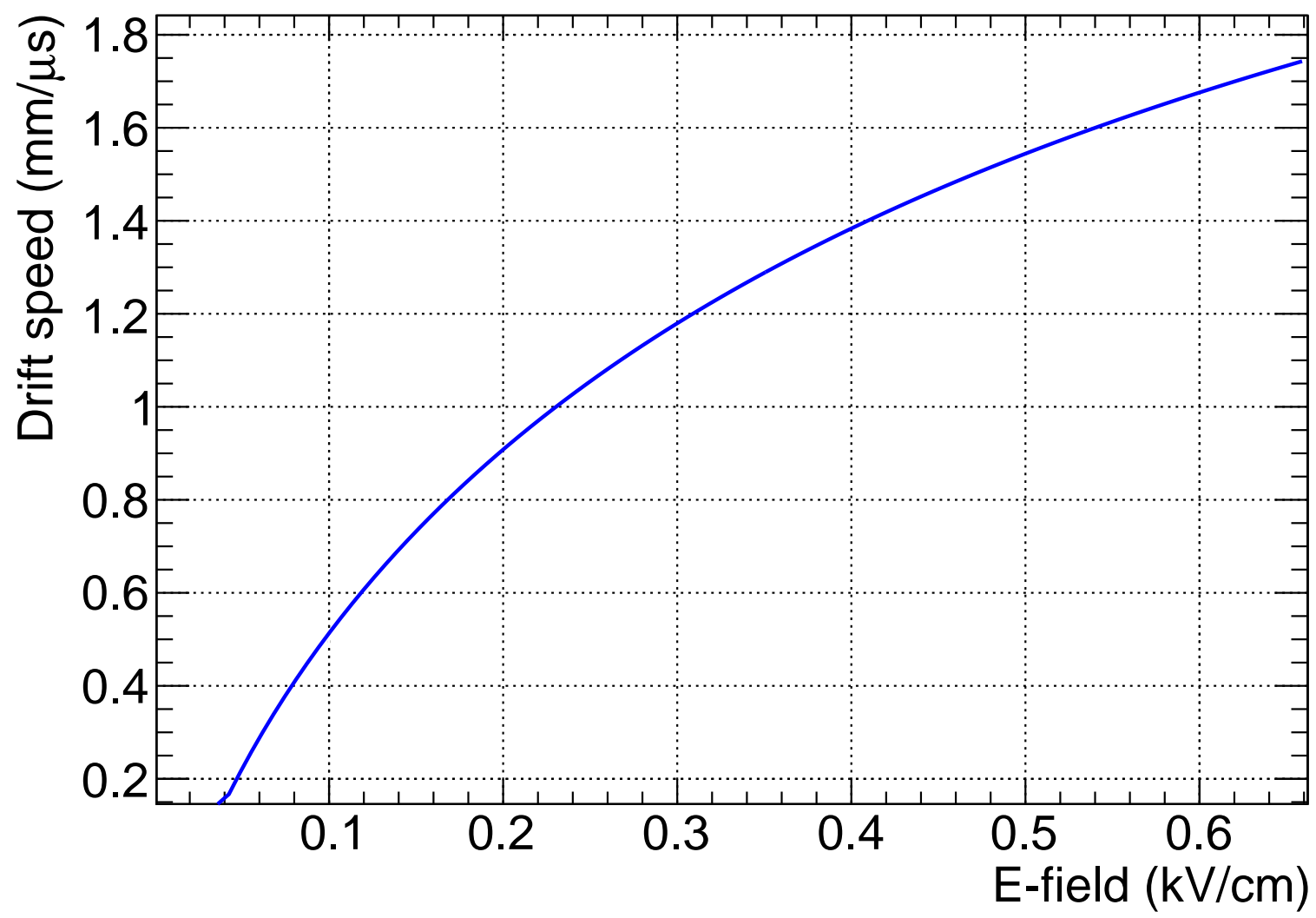

Figure 28. Drift speed as a function of E-field [26, 27], in the range of $0.04 \mathrm{kV} \mathrm{cm}^{-1}$ to $0.66 \mathrm{kV} \mathrm{cm}^{-1}$.

\subsection{Validation of the E-field determination}

The space charge simulation in LArSoft, described in section 4, contains an input E-field map, a correction map and a distortion map. We take the correction map from the space charge simulation to calculate a E-field map. The calculated E-field map is then compared to the simulation truth to evaluate the bias introduced by this method. The bias is defined as the bin-to-bin difference 
of calculated E-field and E-field from the simulation truth. The bias also folds uncertainties of the E-field map and the correction map from the space charge simulation. The E-field map from simulation truth has 21 bins in $X, 21$ bins in $Y$ and 81 bins in $Z$. The first and last bin centers of each direction are located at the TPC limits. To avoid introducing different bias, we use the same binning for the calculated E-field map in validation.

Figures 29 and 30 show the calculated E-field (top) and the E-field from the simulation truth (bottom) at the central $Z$ and an upstream $Z$ slice, respectively. We present $2 \mathrm{D}$ slices of the distortion in the E-field map as a percentage with respect to the magnitude of the nominal E-field $E_{0}=274$ $\mathrm{V} / \mathrm{cm}$ in components, $\left(E_{X}-E_{0}\right) / E_{0}[\%]$ (left), $E_{Y} / E_{0}[\%]$ (middle), $E_{Z} / E_{0}[\%]$ (right). The nominal E-field is a uniform E-field along $X$ in the TPC. At central $Z$, near the cathode, $E_{X}$ is larger than $E_{0}$ by about $9 \%$, and near the anode $E_{X}$ is smaller than $E_{0}$ by about $6 \%$. At central $Z$, the maximum value of $E_{Y}$ is about $15 \%$ of $E_{0}$ and points outwards at the top and the bottom of the TPC. At central $Z, E_{Z}$ is relatively small. The E-field distortion in the $Z$ direction is less than $1 \%$ of $E_{0}$. At an upstream $Z$ slice, near the cathode, $E_{X}$ exceeds $E_{0}$ by about $1.5 \%$ and near the anode $E_{X}$ is below $E_{0}$ by about $1 \%$. The vertical component $E_{Y}$ is about $4 \%$ of $E_{0}$ and points outwards at the top and the bottom of the TPC. The component along the beam, $E_{Z}$, points to the outside of the TPC. The maximum distortion in $E_{Z}$ is about $10 \%$ of $E_{0}$ and the position of the maximum is at $X \sim 160 \mathrm{~cm}$ and $Y \sim 0 \mathrm{~cm}$. The general shape of the calculated E-field distortion agrees well with simulation truth (compare figures 29 and 30).

The bias is the difference between the calculated E-field distortion and the true E-field distortion as introduced in the simulation. Figure 31 shows the bias in $X, Y, Z$ components in $\mathrm{V} \mathrm{cm}^{-1}$. The $Y$ and $Z$ distributions are all narrowly peaked around zero. The bias of $E_{X}$ has a mean value of $0.96 \mathrm{~V} \mathrm{~cm}^{-1}$ and the standard deviation of the bias in the $X, Y$ and $Z$ components are $0.201 \mathrm{~V} \mathrm{~cm}^{-1}$, $0.451 \mathrm{~V} \mathrm{~cm}^{-1}$ and $0.142 \mathrm{~V} \mathrm{~cm}^{-1}$, respectively. Figure 32 shows the bias of the magnitude of the E-field in $\mathrm{V} \mathrm{cm}^{-1}$. The mean and the standard deviation of the bias distribution are $0.95 \mathrm{~V} \mathrm{~cm}^{-1}$ and $0.074 \mathrm{~V} \mathrm{~cm}^{-1}$ respectively. The bias in E-field magnitude is dominated by the bias in $E_{X}$, which is sensitive to the nominal E-field and the drift distance used in the input space charge simulation. The small bias is also visible in figure 29 and 30. This may be caused by our limited knowledge of the cold cathode position. Overall, the bias between the reconstructed and simulated E-fields is negligible compared to the expected E-field distortions, which validates the method of calculating E-field and drift velocity.

\section{Measurements in MicroBooNE from laser data}

Using laser data collected with the MicroBooNE experiment, and the track reconstruction and selection described in section 3, we extract the spatial distortion and correction maps, and the corresponding drift velocity and E-field maps.

\subsection{Distortion maps}

With the method described in section 5, we derive the distortion and correction maps determined from the UV-laser data. We present 2D slices of the 3D maps and the corresponding uncertainties.

Figure 33 shows at central $Z$ the spatial distortion of $d X=X_{\text {reco }}-X_{\text {true }}, d Y=Y_{\text {reco }}-Y_{\text {true }}$ and $d Z=Z_{\text {reco }}-Z_{\text {true }}$ calculated from laser data. The corresponding figures from simulation are 

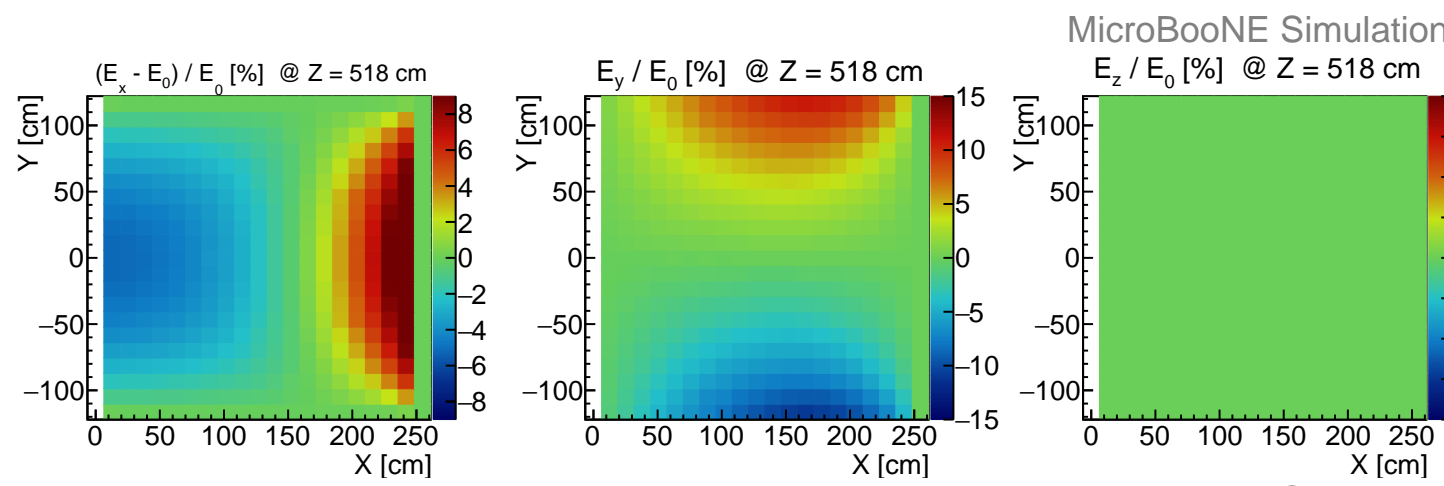

$\mathrm{E}_{\mathrm{z}} / \mathrm{E}_{0}[\%] @ \mathrm{Z}=518 \mathrm{~cm}$

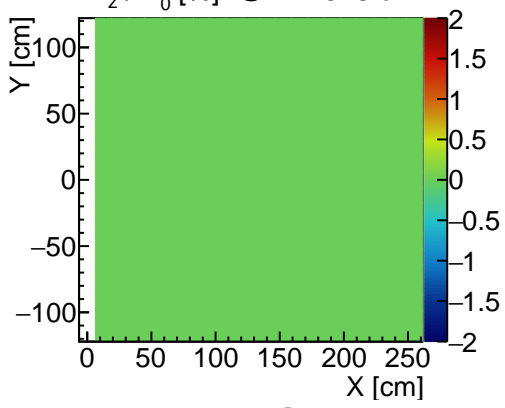

MicroBooNE Simulation
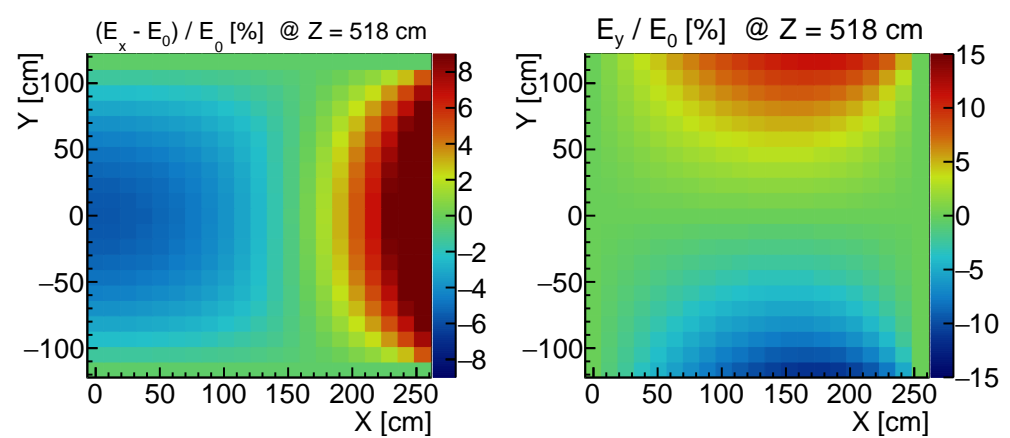

$E_{z} / E_{0}[\%] @ Z=518 \mathrm{~cm}$

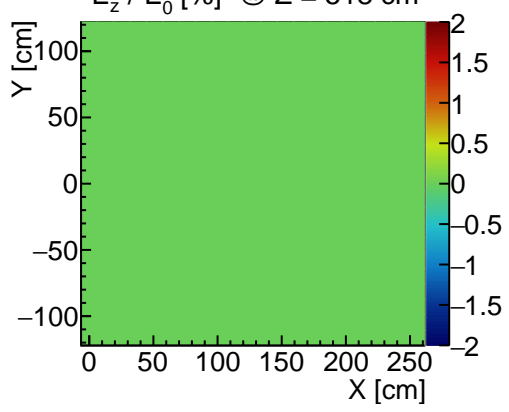

Figure 29. Relative E-field distortions (in percent) with respect to the nominal E-field in components $\left(E_{X}-E_{0}\right) / E_{0}[\%]$ (left), $E_{Y} / E_{0}[\%]$ (middle) and $E_{Z} / E_{0}[\%]$ (right). The upper row shows the result of the calculated E-field from the correction map. The lower row shows the E-field from the space charge simulation, which is used as simulation truth here. Both of them show the E-field distortion at a central slice at $Z=518 \mathrm{~cm}$, in the TPC true spatial coordinates.

Table 2. Spatial distortion measured from laser data for selected representative points in the TPC.

\begin{tabular}{|c|c|c|c|}
\hline Position $(X, Y, Z)[\mathrm{cm}]$ & $d X \pm$ stat. \pm syst. [cm] & $d Y \pm$ stat. \pm syst. [cm] & $d Z \pm$ stat. \pm syst. [cm] \\
\hline$(174.11,-4.65,518.40)$ & $4.22 \pm 0.34 \pm 0.10$ & $-2.00 \pm 0.26 \pm 0.60$ & $-0.01 \pm 0.44 \pm 0.11$ \\
\hline$(30.72,-4.65,518.40)$ & $1.15 \pm 0.02 \pm 0.05$ & $-0.02 \pm 0.52 \pm 0.04$ & $-0.01 \pm 0.06 \pm 0.01$ \\
\hline$(225.32,-60.45,518.40)$ & $1.11 \pm 0.58 \pm 0.37$ & $6.51 \pm 1.04 \pm 0.05$ & $0.09 \pm 0.68 \pm 0.14$ \\
\hline$(225.32,60.45,518.40)$ & $1.46 \pm 0.38 \pm 0.38$ & $-8.20 \pm 0.82 \pm 0.06$ & $-0.01 \pm 0.48 \pm 0.05$ \\
\hline
\end{tabular}

figure 9 and figure 12. The maximal $d X$ observed in data is about $4 \mathrm{~cm}$, located around $X \approx 160 \mathrm{~cm}$ and $Y \approx 0 \mathrm{~cm}$. $d X$ is larger than the simulation and beyond the calculated uncertainty, which indicates that space charge in the simulation may be underestimated. Close to the cathode, the upper and lower edges of $Y$ are distorted by about $15 \mathrm{~cm}$ inwards in the TPC. $d Z$ is relatively small and uniform. The overall shapes of the spatial distortions in data are similar to the simulated shape.

The standard deviation in each bin is calculated from 50 sub-maps as described in section 5.4. The standard deviation for the central $Z$ slice is shown in figure 34 . This is taken as the statistical uncertainty for spatial distortion in each bin. It is of a similar size as the standard deviation calculated from the full laser simulation (figure 13). The systematic uncertainty on data is estimated from the bias of the true and calculated displacement in the full laser simulation (figure 14 in section 5.5.2). 

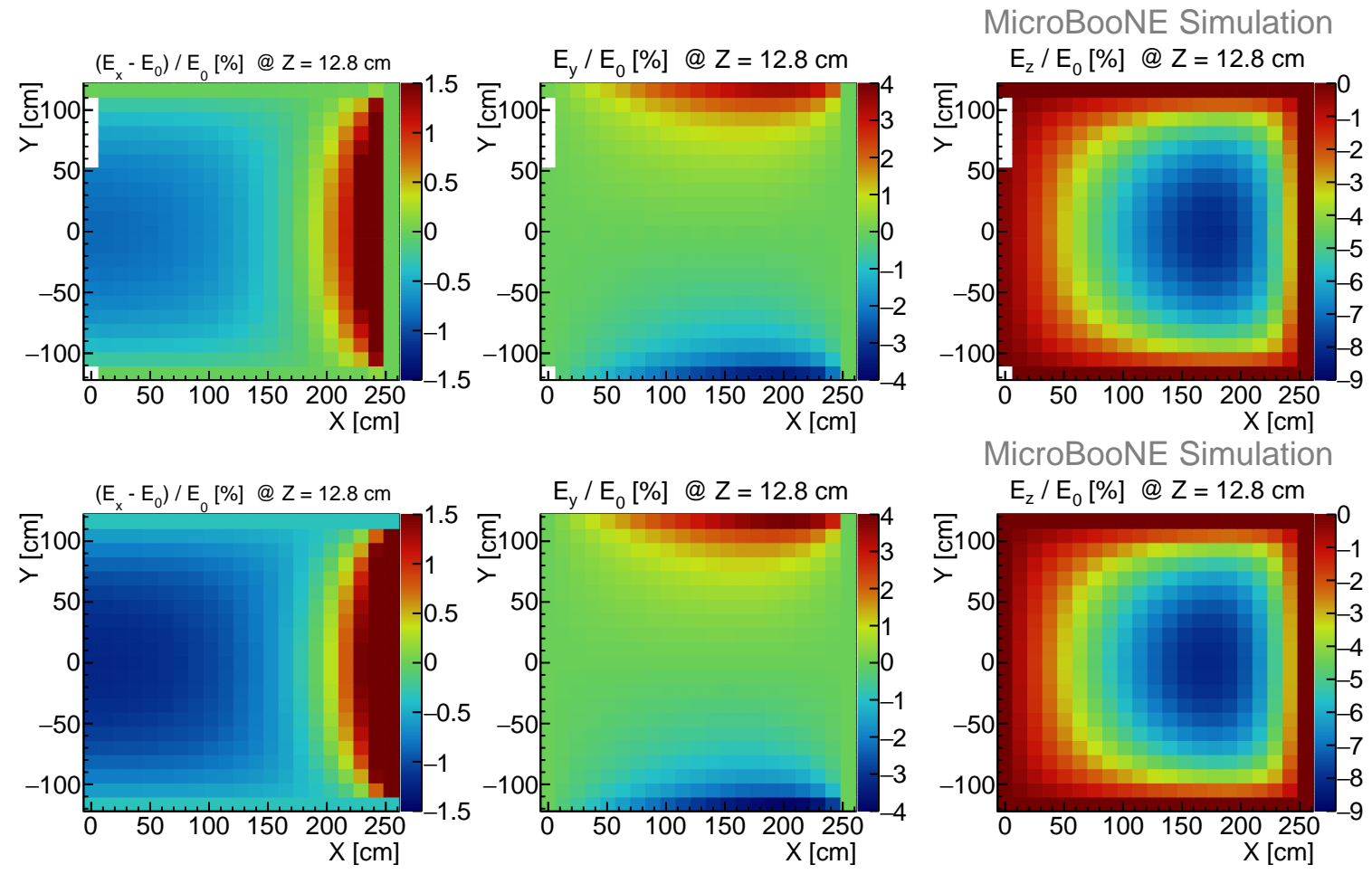

$E_{z} / E_{0}[\%] @ Z=12.8 \mathrm{~cm}$

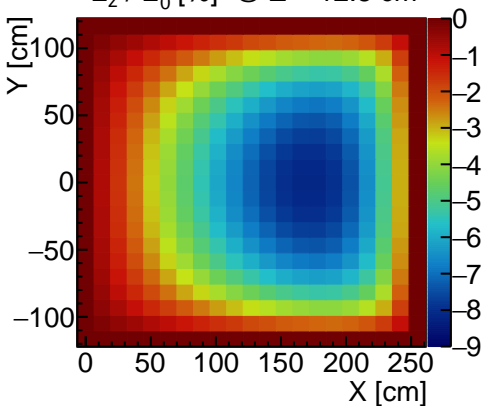

Figure 30. Relative E-field distortion (in percent) with respect to the nominal E-field in components $\left(E_{X}-E_{0}\right) / E_{0}[\%]$ (left), $E_{Y} / E_{0}[\%]$ (middle), $E_{Z} / E_{0}[\%]$ (right). The upper row is the result of the calculated E-field from the correction map. The lower row is the E-field from the space charge simulation, which is used as simulation truth here. Both of them show the E-field distortion at an upstream slice at $Z=12.8 \mathrm{~cm}$ in the TPC true spatial coordinates.
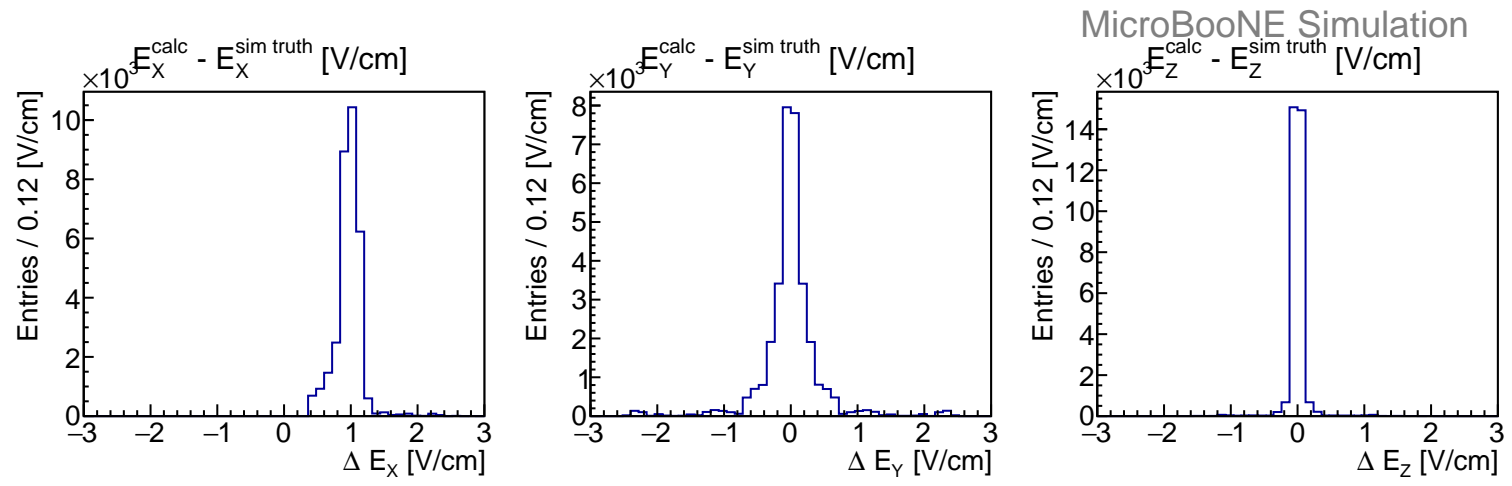

Figure 31. Distributions of bias components $E_{X}^{\mathrm{calc}}-E_{X}^{\text {sim truth }}, E_{Y}^{\mathrm{calc}}-E_{Y}^{\text {sim truth }}$ and $E_{Z}^{\mathrm{calc}}-E_{Z}^{\text {sim truth }}$ in $\mathrm{V} \mathrm{cm}^{-1}$. Each entry of the distribution corresponds to the bias in one bin. The distributions of $\Delta E_{Y}$ and $\Delta E_{Z}$ are narrowly peaked around 0 . Mean of $\Delta E_{X}$ is $0.96 \mathrm{~V} \mathrm{~cm}^{-1}$. The slight bias in $E_{X}$ could be due to the nominal E-field and the drift distance used in the input space charge simulation. 


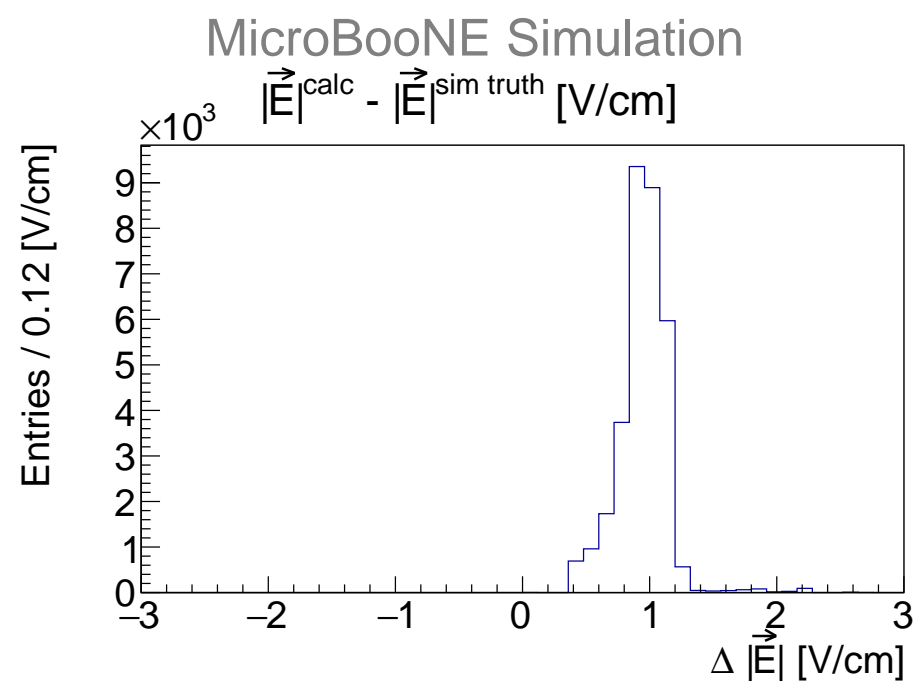

Figure 32. Bias distributions of the local E-field magnitude $|\vec{E}|^{\text {calc }}-|\vec{E}|^{\text {sim truth }}$ in $\mathrm{V} \mathrm{cm}^{-1}$. Each entry of the distribution is the bias in one bin. The calculated E-field is very close to the simulated truth within $0.95 \mathrm{~V} \mathrm{~cm}^{-1}$. The small bias in the local E-field magnitude is dominated by the bias in $E_{X}$.
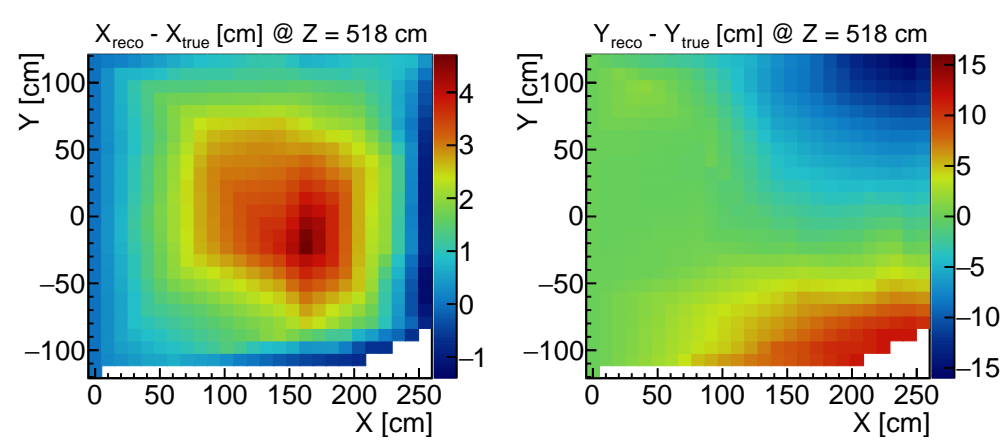

MicroBooNE Laser Data

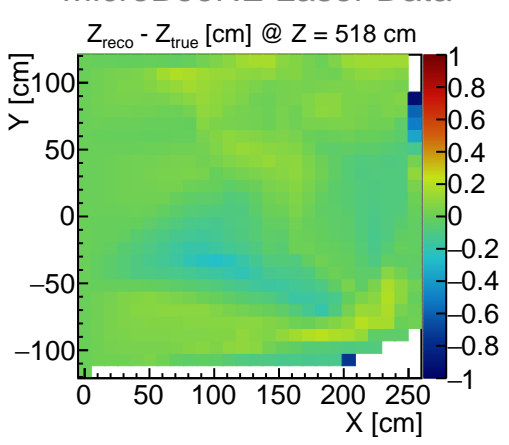

Figure 33. Calculated distortion map from laser data at a central slice in $Z$. Shown are the three components $d X=X_{\text {reco }}-X_{\text {true }}$ (left), $d Y=Y_{\text {reco }}-Y_{\text {true }}$ (middle), and $d Z=Z_{\text {reco }}-Z_{\text {true }}$ (right). In this $Z$ slice the maximum $d X$ is around $4 \mathrm{~cm}$ and the maximum $d Y$ is about $\pm 15 \mathrm{~cm}$. The corresponding figures from simulation are figure 9 and figure 12 .
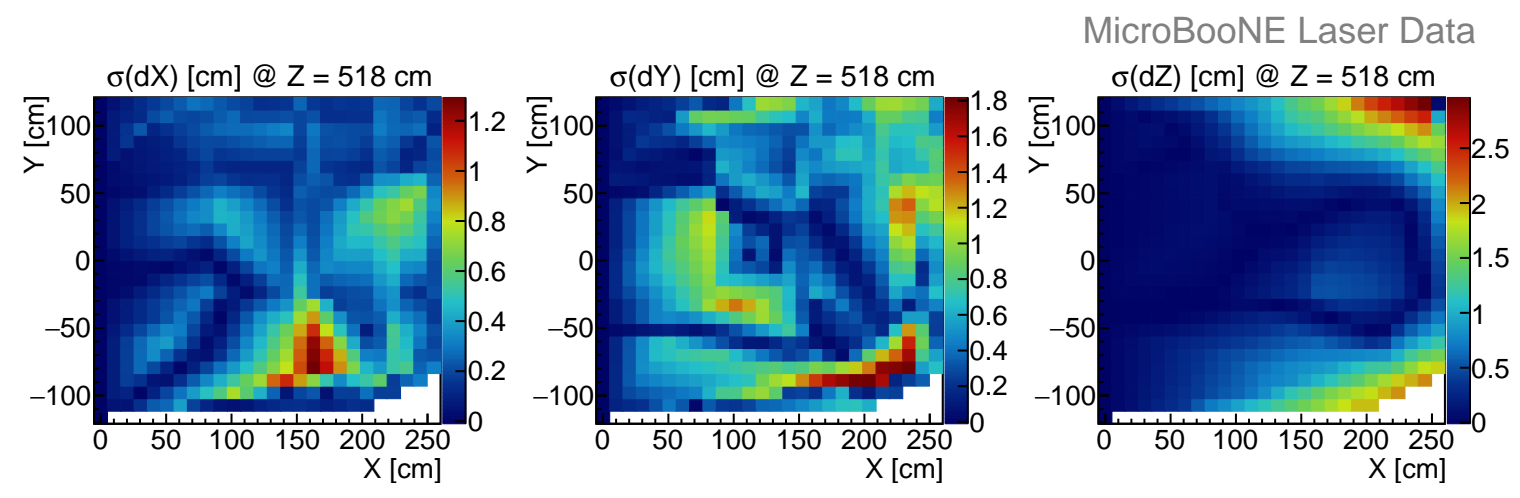

Figure 34. Standard deviations $(\sigma)$ of the spatial distortion $d X, d Y, d Z$ corresponding to figure 33 . 
Selected spatial distortion values, with their uncertainties, are listed in table 2. Given the scale of the spatial distortions, it is crucial to correct for them in order to achieve more accurate tracking and calorimetric information from the TPC.

\subsection{Drift velocity map from laser data}

We calculate the local drift velocity from the correction map, which is based on laser calibration data, as described in section 6.2.

Figure 35 shows the drift velocity components with respect to the nominal drift velocity $v_{0}$. In MicroBooNE, $v_{0}$ is $1.098 \mathrm{~mm} \mathrm{\mu s}^{-1}$. The nominal drift velocity is estimated by looking at the end of cathode-piercing tracks and measuring the drift time for the ionization electrons to travel from the cathode plane to the anode plane. The uncertainty on $v_{0}$ comes from the determination of the cold cathode position and the residual space charge effect at the cathode.
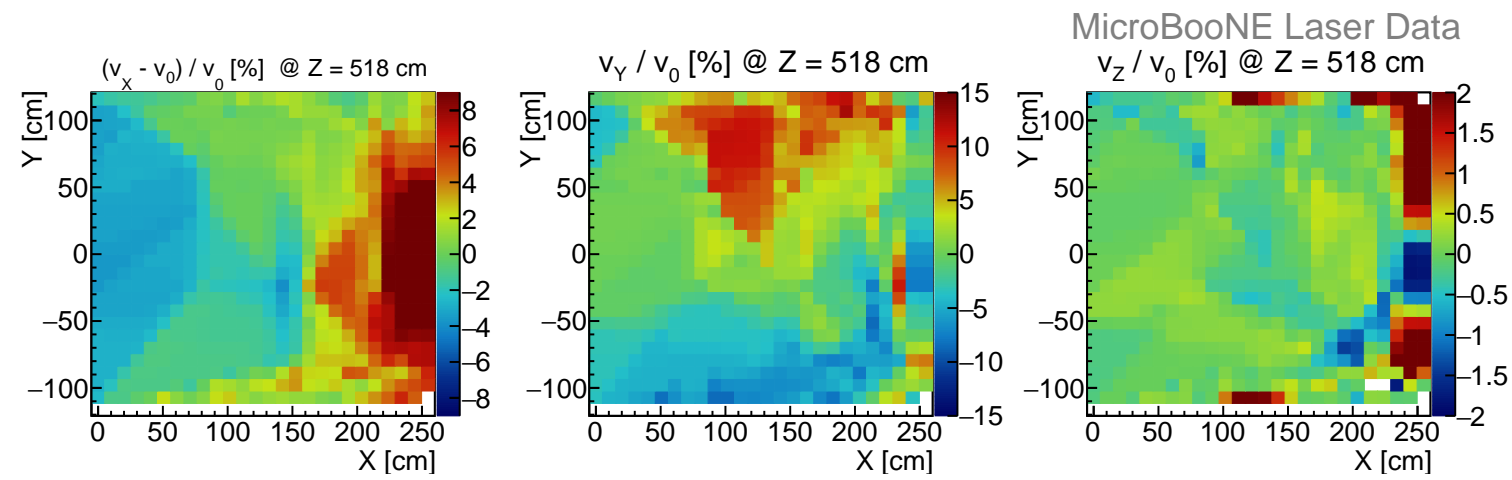

Figure 35. Relative drift velocity components (in percent)for the three coordinates $\left(v_{X}-v_{0}\right) / v_{0}[\%]$ (left), $v_{Y} / v_{0}[\%]$ (middle), and $v_{Z} / v_{0}[\%]$ (right) at a central slice in $Z$. The drift velocity has a maximal distortion from the nominal drift velocity $v_{0}$ of about $10 \%$.

At central $Z$, the drift velocity component along $X$ is about $10 \%$ larger than $v_{0}$ near the cathode and $v_{X}$ is about $6 \%$ smaller than $v_{0}$ near the anode. The area with larger $v_{X}$ is slightly smaller than the area with smaller $v_{X}$. In the upper and lower region, $v_{Y}$ is about $0.1 \mathrm{~mm}^{-1} \mathrm{~s}^{-1}$ pointing outwards. The vertical drift velocity, $v_{Z}$, is relatively small and uniform at central $Z$.

The statistical uncertainty of the drift velocity is derived from the correction map. For each bin of the correction map, 500 correction vectors are generated according to a normal distribution with $\mu$ the central value of the correction vector and $\sigma$ the standard deviation, to produce 500 correction maps. We then propagate these 500 correction maps into velocity maps and in each bin we obtain 500 velocity components. We take the standard deviation from a fit to the distributions in each bin as the statistical uncertainty of the velocity measurement. As we are eventually interested in the E-field, more details on the uncertainties are given in section 6.3.

The systematic uncertainty of the local drift velocity is again determined with a bias study using the full laser simulation. The bias is the difference between the calculated drift velocity and the drift velocity from the input simulation. The calculated drift velocity is taken from the correction map estimated using the full laser simulation. The drift velocity in simulation is the drift velocity taken from the relationship shown in figure 28, corresponding to the E-field from the space charge 
Table 3. Representative local drift velocities measured using MicroBooNE laser data. In all cases, the uncertainty is dominated by statistical uncertainty, which is at least an order of magnitude larger than the systematic uncertainty.

\begin{tabular}{|c|c|c|c|}
\hline Position $(X, Y, Z)[\mathrm{cm}]$ & $v_{X} \pm$ stat. $\left[\mathrm{mm} \mu \mathrm{s}^{-1}\right]$ & $v_{Y} \pm$ stat. $\left[{\left.\mathrm{mm} \mu \mathrm{s}^{-1}\right]}^{-1}\right.$ & $v_{Z} \pm$ stat. $\left[\mathrm{mm} \mu \mathrm{s}^{-1}\right]$ \\
\hline$(30.58,-4.65,518.40)$ & $1.062 \pm 0.001$ & $0.000 \pm 0.014$ & $0.000 \pm 0.001$ \\
\hline$(224.22,-4.65,518.40)$ & $1.197 \pm 0.015$ & $0.002 \pm 0.008$ & $-0.004 \pm 0.016$ \\
\hline$(122.30,-79.05,518.40)$ & $1.086 \pm 0.013$ & $-0.046 \pm 0.005$ & $0.001 \pm 0.006$ \\
\hline$(122.30,79.05,518.40)$ & $1.101 \pm 0.001$ & $0.123 \pm 0.008$ & $0.002 \pm 0.010$ \\
\hline
\end{tabular}

simulation. A set of representative resulting drift velocities and uncertainties measured with laser data is listed in table 3 .

\subsection{E-field map measured from laser data}

We calculate the absolute local E-field from the correction map derived from laser data as described in section 6.3.

Figure 36 shows the components of the E-field map relative to the nominal E-field at central $Z:\left(E_{X}-E_{0}\right) / E_{0}[\%], E_{Y} / E_{0}[\%]$ and $E_{Z} / E_{0}[\%]$. The nominal E-field in MicroBooNE is $E_{0}=$ $273.9 \mathrm{~V} \mathrm{~cm}^{-1}$ along the drift direction $X$, which is derived from the high voltage applied between the cathode and the anode and the estimated drift length in liquid argon.

At central $Z$, the E-field component along the drift direction $X$ is about $10 \%$ larger than $E_{0}$ near the cathode and is about $6 \%$ smaller than $E_{0}$ near the anode. The area with weaker $E_{X}$ is slightly larger than the area with stronger $E_{X}$. In the upper region, $E_{Y}$ is about $30 \mathrm{~V} \mathrm{~cm}^{-1}$ pointing upwards. In the lower region, $E_{Y}$ is about $15 \mathrm{~V} \mathrm{~cm}^{-1}$ pointing downwards. $E_{Z}$ is relatively small and flat at central $Z$, mostly within -2 and $+2 \mathrm{~V} \mathrm{~cm}^{-1}$. The lowest row in $Y$ is empty due to lack of laser coverage.

The dominant contribution to the E-field distortion in MicroBooNE is space charge arising from cosmic rays. Muons from cosmic rays enter the TPC at a rate of $\sim 10 \mathrm{kHz}$. Positive charge, which drifts $\sim 10^{5}$ times slower than the electrons in the same E-field, builds up in the form of an ion cloud near the cathode. If ionization electrons travel through the ion cloud from the cathode side, they will be boosted by the E-field from the attraction of the ion cloud. Along the $X$ direction, the E-field near the cathode plane is typically larger than the nominal E-field. If ionization electrons travel in the ion cloud, they are attracted from the ion cloud from both the $+X$ and $-X$ directions. At the position where the attraction reaches equilibrium in the drift direction, $E_{X}$ is close to $E_{0}$. As the ionization electrons travel further towards the anode, the attraction from the ion cloud pulls the electrons backwards. This reduces the total $E_{X}$ close to the anode due to the static E-field produced by the ion cloud in the opposite direction to $E_{0}$. Similarly, ionization electrons are attracted to the ion cloud in all directions, and indeed we observe $E_{Y}$ pointing outwards in the upper and lower regions of the TPC. The asymmetry of $E_{Y}$ in the upper and lower regions may be due to the cosmic ray muons entering the TPC at the top, with fewer ions created at the bottom. Additionally the poor laser coverage near the bottom of the TPC may contribute to this. Furthermore, convection of the liquid argon inside the TPC can move ion charges. The E-field along the beam direction $E_{Z}$ is very 
small and uniform, because the space charge distribution is almost symmetric with respect to the central $Z$.
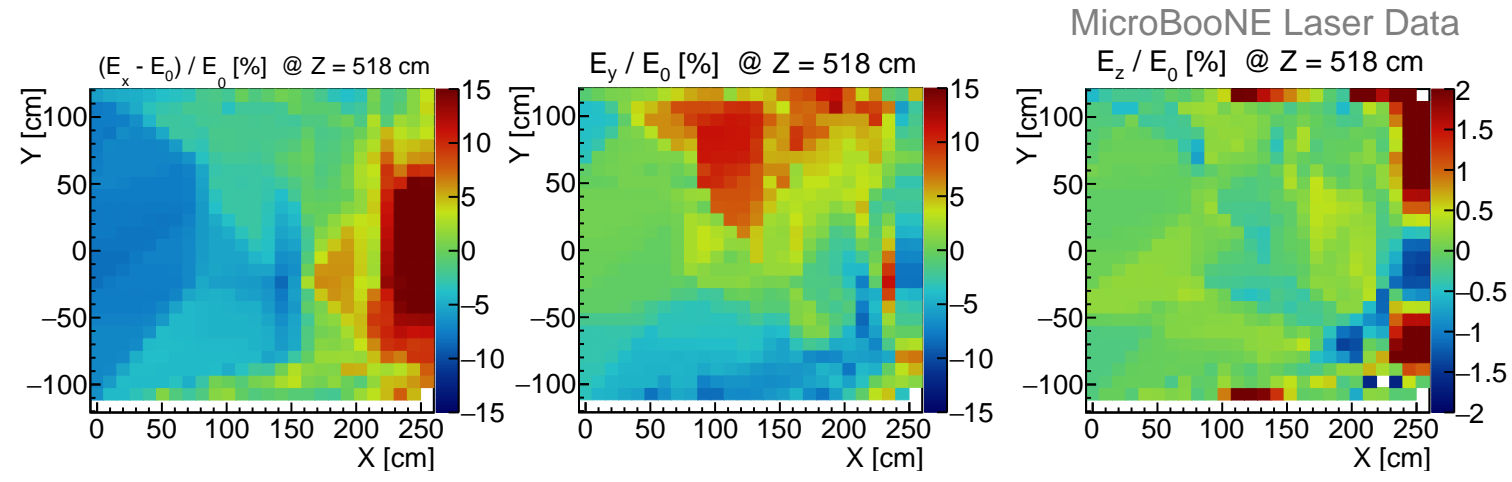

Figure 36. Relative E-field components (in percent) for the three coordinates $\left(E_{X}-E_{0}\right) / E_{0}[\%]$ (left), $E_{Y} / E_{0}[\%]$ (middle) and $E_{Z} / E_{0}[\%]$ (right) at a central slice in $Z$. The maximal relative E-field distortion is about $10 \%$.

The statistical uncertainty of the measured E-field obtained using laser data is calculated by propagating the statistical uncertainty from the correction map in the same way as was done for the velocity map (section 7.2). An example of the distribution of E-field component in a bin can be seen in figure 37 . We take the standard deviation from a fit to the distribution in every bin as the statistical uncertainty of the E-field measurement. In figure 28, around the nominal E-field in MicroBooNE $E_{0}$, and with the E-field distortion up to $50 \mathrm{~V} \mathrm{~cm}^{-1}$, the drift speed varies almost linearly with respect to the magnitude of the E-field. As the error propagation to the E-field shows Gaussian distributions in figure 37 we use the values from the fit.

The statistical uncertainty shown as a percentage of $E_{0}$ at central $Z$ is shown in figure 38 . Typical statistical uncertainties of the E-field component in a bin are less than $2 \%$ of $E_{0}$.

The systematic uncertainty of the E-field measurement performed with laser data is also determined by the bias study using the full laser simulation. The bias is defined as the difference between the simulated E-field (section 6.4) and the calculated E-field using the full laser simulation described in section 5.5.2. The E-field in the simulation is derived from the correction map, based on the space charge simulation, and is binned in $(21,21,81)$ bins in $(X, Y, Z)$. We interpolate the simulated E-field map in the same binning as the calculated one. The deviations that we observe at the edge of the map are due to the tri-linear interpolation lacking surrounding cubes. This systematic uncertainty, shown as a percentage of the nominal E-field $E_{0}$ at central $Z$, is shown in figure 39. Typical systematic uncertainties in a bin are $-2 \%$ to $2 \% E_{0}$.

A set of representative E-field vectors measured by laser data is listed in table 4 . The values are presented as relative E-field distortions in components of $\Delta E_{X}=E_{X}-E_{0}, \Delta E_{Y}=E_{Y}, \Delta E_{Z}=E_{Z}$. The E-field distortion reaches $\sim 30 \mathrm{~V} \mathrm{~cm}^{-1}$, with typical uncertainties of a few $\mathrm{V} \mathrm{cm}^{-1}$.

\section{Time stability studies}

The time stability of the E-field in the TPC is important in order to estimate the required frequency of the E-field measurements. It also provides an assessment of additional uncertainties of the E-field measurement for a single measurement which are not included in section 6.3. 


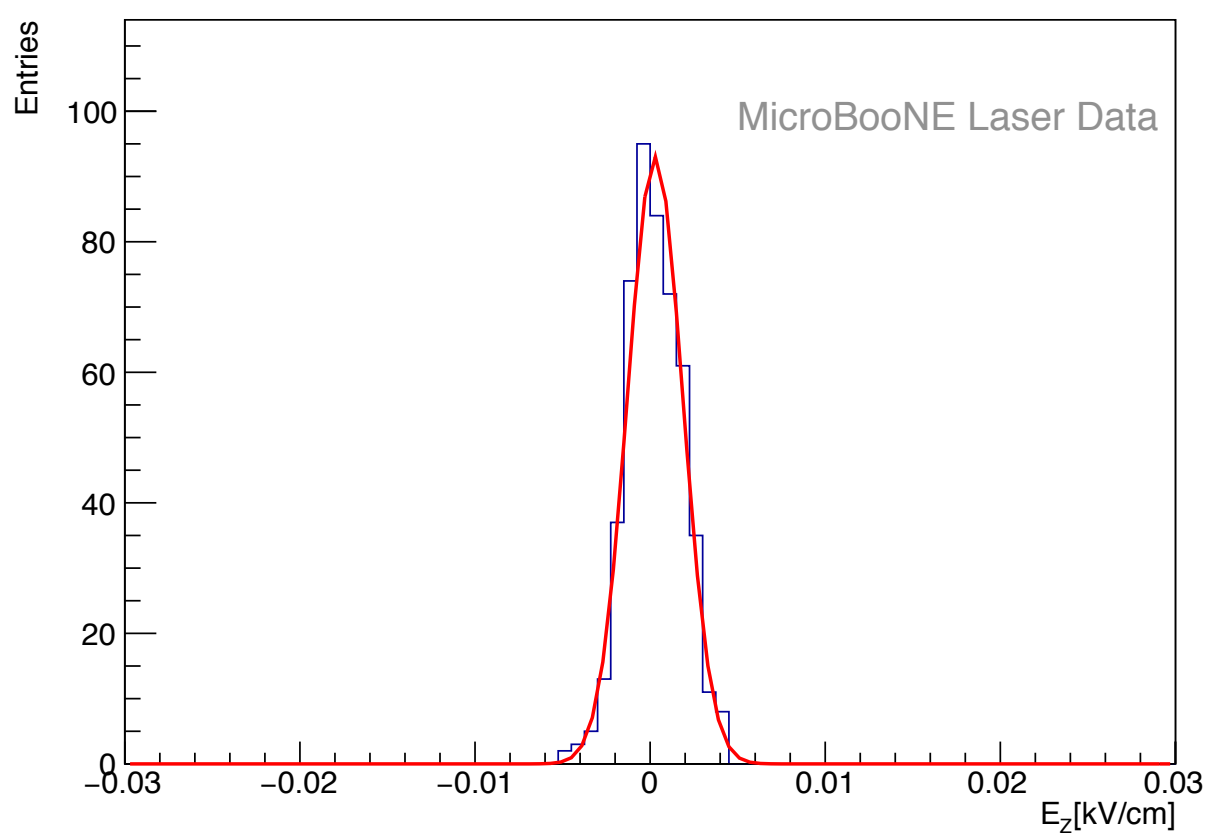

Figure 37. Distribution of the vertical E-field components $E_{Z}$ for 500 initial correction maps used in the error propagation. The red line shows the Gaussian fit.
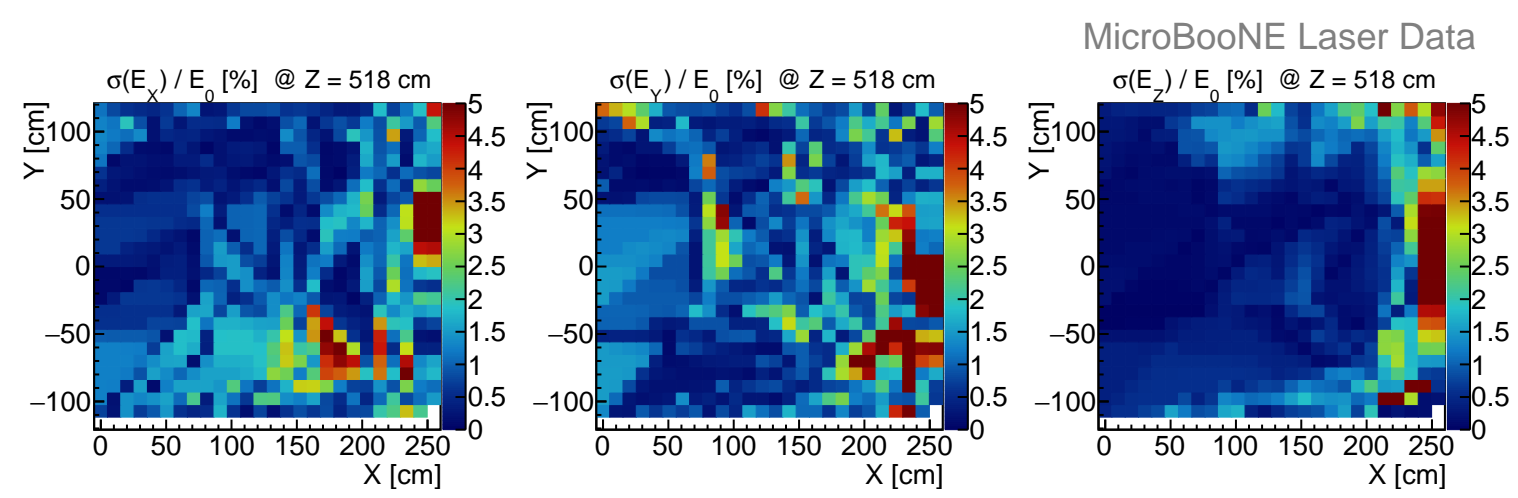

Figure 38. Standard deviation of the E-field components in each bin with respect to the nominal E-field in a central slice in $Z$. From left to right the three components are shown: $\sigma\left(E_{X}\right) / E_{0}[\%], \sigma\left(E_{Y}\right) / E_{0}[\%]$ and $\sigma\left(E_{Z}\right) / E_{0}[\%]$. The deviations are mostly within $2 \%$ of the nominal E-field $E_{0}$, with the exception of some bins. Here the standard deviation of the E-field in each bin is the statistical uncertainty of the E-field measurement with laser data.

Table 4. Representative E-field distortions measured by laser data.

\begin{tabular}{|c|c|c|c|}
\hline Position $(X, Y, Z)[\mathrm{cm}]$ & $\Delta E_{X} \pm$ stat. \pm syst. $\left[\mathrm{V} \mathrm{cm}^{-1}\right]$ & $\Delta E_{Y} \pm$ stat. \pm syst. $\left[\mathrm{V} \mathrm{cm}^{-1}\right]$ & $\Delta E_{Z} \pm$ stat. \pm syst. $\left[\mathrm{V} \mathrm{cm}^{-1}\right]$ \\
\hline$(30.58,-4.65,518.40)$ & $-21.24 \pm 0.25 \pm 1.06$ & $-0.04 \pm 3.31 \pm 0.06$ & $0.19 \pm 0.21 \pm 0.01$ \\
\hline$(224.22,-4.65,518.40)$ & $34.21 \pm 6.85 \pm 1.09$ & $0.51 \pm 2.19 \pm 0.31$ & $-0.93 \pm 4.18 \pm 0.01$ \\
\hline$(122.30,-79.05,518.40)$ & $-11.83 \pm 4.89 \pm 1.29$ & $-11.03 \pm 1.11 \pm 1.33$ & $0.21 \pm 1.49 \pm 0.03$ \\
\hline$(122.30,79.05,518.40)$ & $-5.28 \pm 0.60 \pm 0.29$ & $30.01 \pm 2.06 \pm 0.17$ & $0.37 \pm 2.50 \pm 0.72$ \\
\hline
\end{tabular}



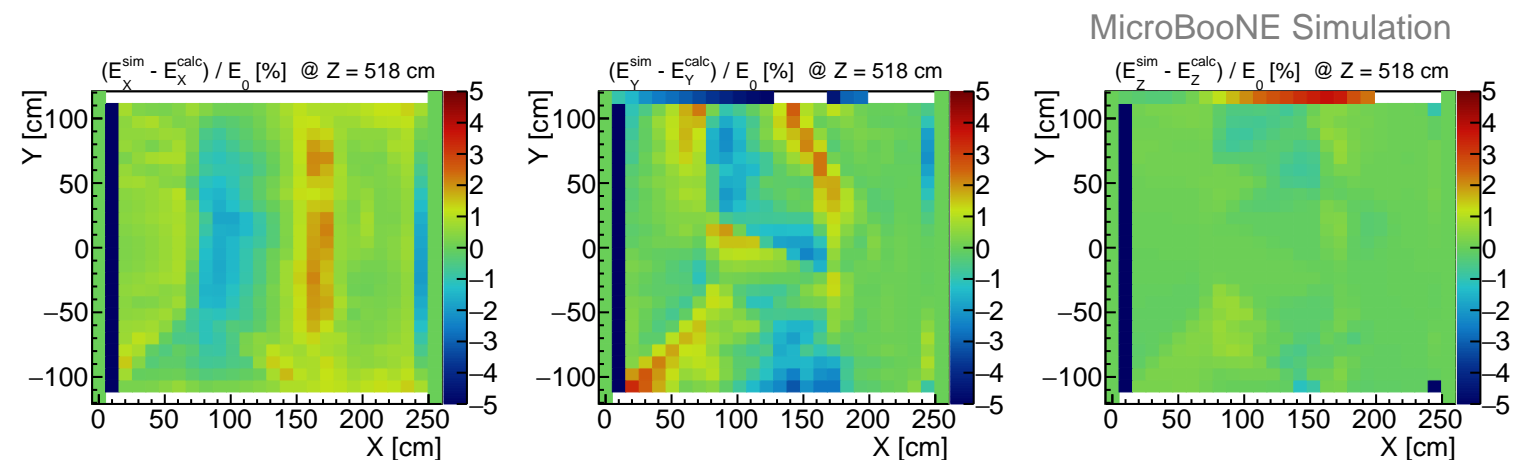

Figure 39. Bias of E-field components as determined from the full laser simulation in percent of the nominal E-field at a central slice in $Z$. From left to right the three coordinates are shown: $\left(E_{X}^{\text {sim }}-E_{X}^{\text {calc }}\right) / E_{0}[\%]$, $\left(E_{Y}^{\text {sim }}-E_{Y}^{\text {calc }}\right) / E_{0}[\%]$ and $\left(E_{Z}^{\text {sim }}-E_{Z}^{\text {calc }}\right) / E_{0}[\%]$. The bias is mostly within $2 \%$ of $E_{0}$. The bias of the E-field determined by the full laser simulation in each bin is used as systematic uncertainty for the E-field measurement with laser data.

A time stability study of the E-field over longer time scales of months or years will be part of an upcoming publication. In this section, we report the results of a time stability study performed over a few hours. One of the major contributions to the E-field distortion in MicroBooNE is charge build-up from cosmic induced ions. The positive charges induced by a single cosmic ray muon, which enters the MicroBooNE detector, typically stay in the TPC for $O(\min )$. Furthermore, the LAr is constantly circulated through filters to keep the concentration of electronegative impurities low, and additionally, there is convection through heat input through the cryostat. These convections could possibly move the charges in the LAr.

To investigate the stability of the E-field, we pulse the laser with the mirror set to point at a constant direction across the TPC in $Z$ over a few hours. The frequency of the laser beam pulse is kept low, at $\sim \mathrm{mHz}$, so multi-photon ionization from the laser would not contribute to space charge. To avoid the possible uncertainties from laser track reconstruction, explained in section 3.1, we conduct the time stability study by looking at the raw waveform signals from the wire readout. If the E-field varies over time, the position (time in the waveform) of the charge deposition of the laser in the TPC also changes due to variations in the drift velocity and path. Thus, the position of the laser induced waveforms in each wire over time is a good representation of the time stability.

We observe the amplitude and the sampling time of the laser signals (peak) in the waveform, where the amplitude is measured in ADC counts and the time in sample ticks. From each raw waveform, an ADC baseline, calculated for that waveform, is subtracted. The ADC unit is then converted to $\mathrm{mV}$ by a known calibration factor. The sample ticks are transformed to $\mu$ s using the fact that every ADC sampling time takes $500 \mathrm{~ns}$. The sampling time of the laser signals is taken with respect to the laser-trigger time, given by a diode in the laser boxes and recording the laser pulse emission. For this time stability study, only waveform signals from the collection plane are used.

First we show the variation of the peak sampling time from three typical wires. The middle plot in figure 40 shows the overlap of 1500 laser pulses from downstream to upstream (right to left). The three wires correspond to different regions in the TPC. The top plot of figure 40 shows one representative waveform out of the 1500 laser events from each chosen wire. The baselines of these 
three waveforms have already been subtracted. As can be seen in the figure, the peak amplitudes of the laser signal in these three wires are similar. The slightly smaller peak amplitude of the first wire is due to the fact that it represents a signal from the laser beam after it traveled in LAr for more than $10 \mathrm{~m}, 3.75 \mathrm{~m}$ more than the next selected wire. The waveforms are fit by Gaussian functions and the mean of the Gaussian is defined as the peak sampling time. The bottom plot of figure 40 shows the histograms of peak sampling times in each of the selected wires. Each histogram only contains peak sampling times of the waveforms from a single wire. The peak sampling time in the top plot contributes to one entry in the corresponding histogram. We then perform a further Gaussian fit to each histogram and obtain $\mu_{1}=943.63 \mu \mathrm{s}, \sigma_{1}=1.52 \mu \mathrm{s}, \mu_{2}=974.92 \mu \mathrm{s}, \sigma_{2}=1.83 \mu \mathrm{s}$, $\mu_{3}=993.95 \mu \mathrm{s}$, and $\sigma_{3}=1.70 \mu \mathrm{s}$ for the three wires, respectively. The width, $\sigma$, of the Gaussian distribution indicates the spread in laser position in the TPC, which reflects the stability of the E-field. In these studies the stability of the laser track itself can be neglected due to mechanical constraints and considerations.

Among all the wires of the collection plane, the largest variation from the Gaussian fit of the waveforms is found to be $\sigma_{\max }=1.93 \mu \mathrm{s}$. On that wire, the best fit gives $\mu=956.23 \mu \mathrm{s}$, corresponding to a spread of the laser position on a single wire of $\sigma_{\max }=2.2 \mathrm{~mm}$. This implies a $0.2 \%$ change in drift velocity along the $X$ direction $\left(v_{X}\right)$ and a time-related variation of the E-field of less than $0.3 \%$.

Figure 41 shows the peak sampling time of the laser signal for more wires over time. The horizontal axis is the laser triggering time with respect to the first laser pulse. The vertical axis shows the relative peak sampling time for the different wires. For simplicity, we skip 100 wires $(\sim 30 \mathrm{~cm})$ in between every wire that is shown. The baseline of the peak sampling time is incremented by $10 \mu$ s from the previous wire that is shown for visibility. The pattern of the peak sampling time over the presented 2 hour range shows correlations with respect to the neighboring wires in time. This implies that the time-related variations are position dependent. Thus, the variations in peak sampling time are likely to be indeed related to the E-field.

In the area with the largest distortion we find variations along $X$ of about $2 \mathrm{~mm}, 0.004 \mathrm{~mm} \mathrm{ss}^{-1}$ and $1.8 \mathrm{~V} \mathrm{~cm}^{-1}$ in spatial displacement, drift velocity and E-field respectively. This is not considered to be significant and thus the E-field in the TPC is found to be stable over a few-hours timescale. 

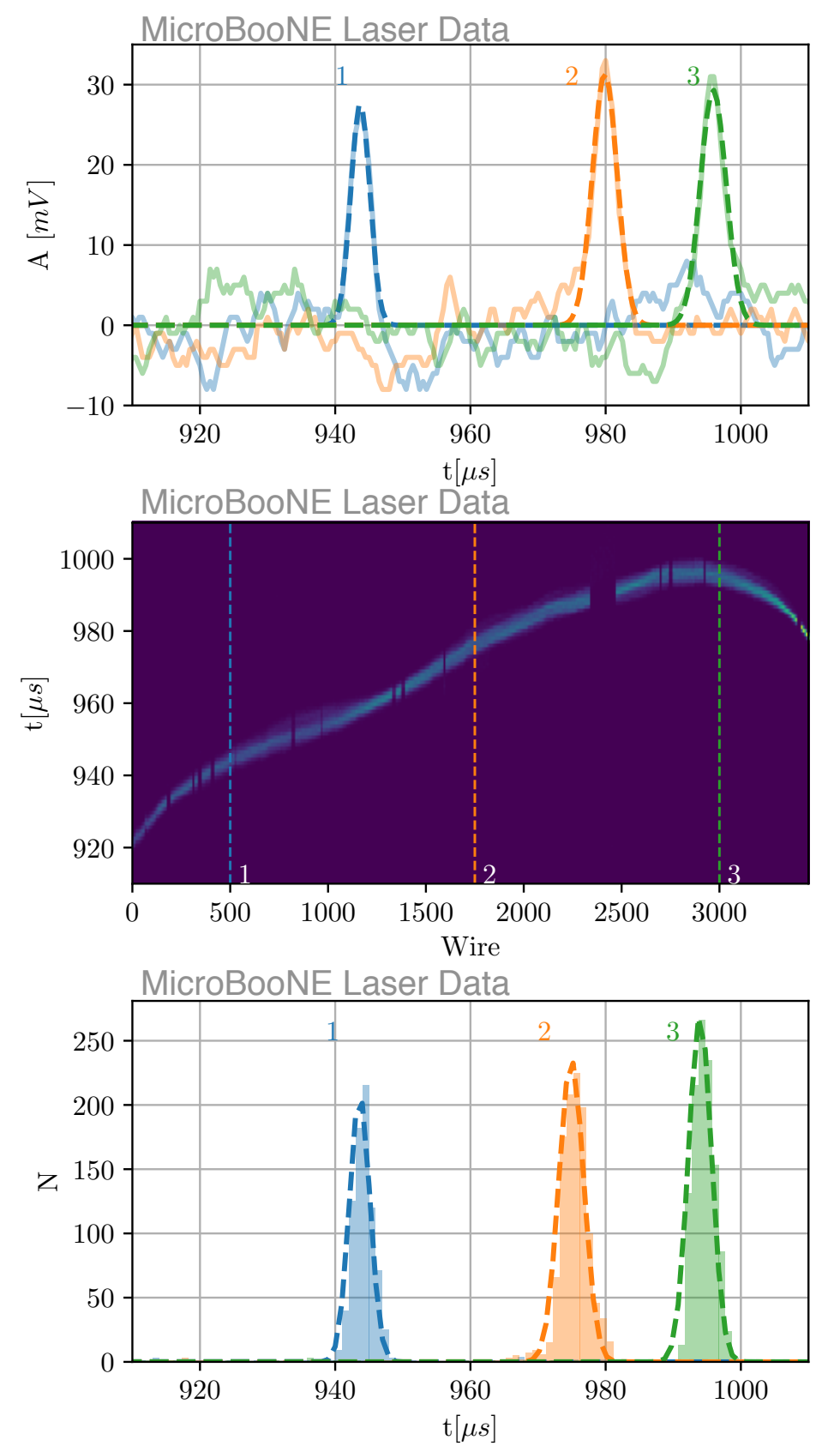

Figure 40. Top: Example of raw waveforms of selected wires in an event: wire 500 (blue, labeled as case 1), wire 1750 (orange, labelled as case 2) and wire 3000 (green, labelled as case 3). Each waveform is fitted by a Gaussian and the central value of the Gaussian defines the peak sampling time. Middle: Overlap of 1500 laser pulses. The tracks are nearly straight with a vertical displacement of about $6.6 \mathrm{~cm}$ over a track lengths of $10.4 \mathrm{~m}$. The horizontal axis in the figure is the wire number and the vertical axis is the drift time. The color illustrated is the sum of amplitudes of the 1500 waveforms. Gaps in the color stream (laser beam) correspond to unresponsive wires in the MicroBooNE read-out. The positions of the three wires 1,2 and 3 are indicated in the plot. Bottom: Histogram of peak sampling times on wire 1, 2 and 3. Each histogram has 1500 entries and each entry is the peak sampling time of a waveform on that wire from a laser pulse. Gaussian fits to each histogram result in means and standard deviations of $\mu_{1}=943.63 \mu \mathrm{s}, \sigma_{1}=1.52 \mu \mathrm{s}$, $\mu_{2}=974.92 \mu \mathrm{s}, \sigma_{2}=1.83 \mu \mathrm{s}$ and $\mu_{3}=993.95 \mu \mathrm{s}, \sigma_{3}=1.70 \mu \mathrm{s}$. 


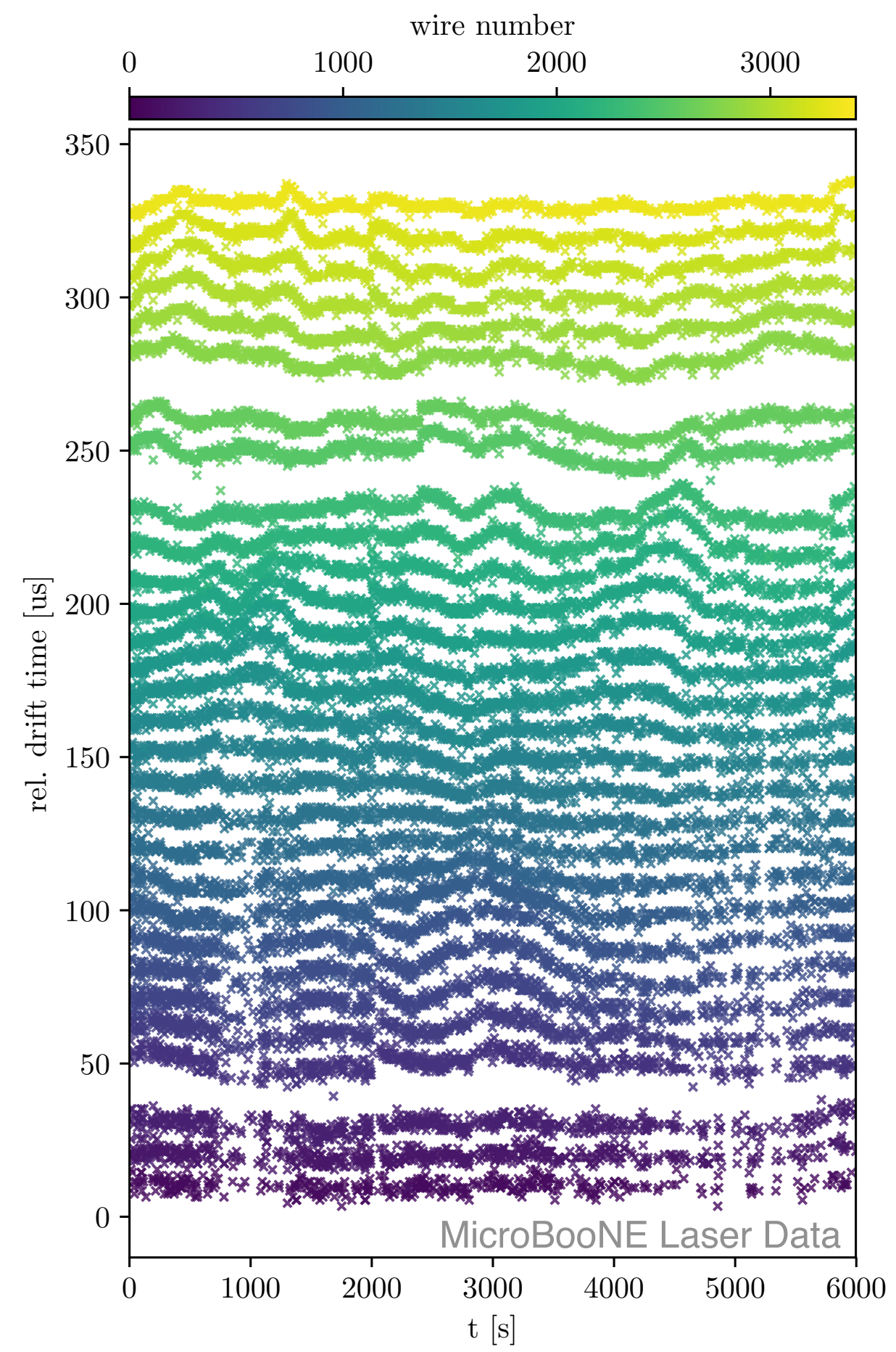

Figure 41. Time evolution of the peak sampling time on different wires. The horizontal axis is the time with respect to the first laser pulse. The vertical axis shows the relative peak sampling time of different wires. The same color indicates that peak sampling time is from the same wire. We skip 100 wires in between every wire that is shown. For display reasons, the baseline of the peak sampling time on each displayed wire are increased by $10 \mu$ s recursively. 


\section{Conclusion}

It is vital for large LArTPCs, such as MicroBooNE and the future SBND and DUNE experiments, to have precise E-field measurements to minimize uncertainties in neutrino event reconstruction, since the E-field of the TPC directly impacts particle tracking, calorimetry, and light yield.

For the first time in a large LArTPC, MicroBooNE has successfully implemented a fully steerable laser system, which can be operated remotely, and has developed a methodology to extract the local E-field, as well as the local drift velocity and spatial displacement, in the TPC active volume. The charge induced by the laser system is an excellent source to examine the TPC readout system. We have observed laser tracks longer than $10 \mathrm{~m}$ long spanning the length of the TPC.

By introducing laser beams along known paths, the spatial displacement of their reconstructed positions affected by E-field distortions can be measured. We determine the E-field map of the MicroBooNE TPC by retracing the drift path of ionization electrons in the TPC E-field and comparing the derived space points to those reconstructed using a nominal E-field. The measured E-field distortion reaches $\sim 30 \mathrm{~V} \mathrm{~cm}^{-1}$ with respect to the nominal field of $273.9 \mathrm{~V} \mathrm{~cm}^{-1}$, and the uncertainties are less than a few $\mathrm{V} \mathrm{cm}^{-1}$. The measured maximal spatial displacement is $\sim 15 \mathrm{~cm}$, and the uncertainties are typically less than $1 \mathrm{~cm}$. In general, the shapes of distortions match the predicted distortions from space charge induced by cosmic ray muons, which is expected to be the largest contribution. Additionally, a time stability study showed that the E-field distortion is stable over a time scale of a few hours.

With the laser setup in MicroBooNE, we measure the E-field, drift velocity and spatial displacement accurately in the bulk of the TPC, including throughout the fiducial volume used for most analyses. The methods established in this work could be adopted by future experiments. The results of these measurements are used to calibrate the position and scale of charge depositions in the MicroBooNE TPC. Using the E-field measurement, we are able to correct for the effect of charge recombination and disentangle it from the correction needed for the electron life time. A good understanding of the drift velocity and E-field, as provided by this work, are essential to understand the impact of charge diffusion in a LArTPC. The low level improvements to the charge calibration provided by this work will help to improve the reconstruction of track length, track direction, measurements of momentum by multiple Coulomb scattering and the vertex of neutrino interactions. These improvements will all improve the performance of particle identification algorithms in MicroBooNE. Future MicroBooNE analyses will use the E-field and spatial calibration based on this work.

\section{Acknowledgments}

This document was prepared by the MicroBooNE collaboration using the resources of the Fermi National Accelerator Laboratory (Fermilab), a U.S. Department of Energy, Office of Science, HEP User Facility. Fermilab is managed by Fermi Research Alliance, LLC (FRA), acting under Contract No. DE-AC02-07CH11359. MicroBooNE is supported by the following: the U.S. Department of Energy, Office of Science, Offices of High Energy Physics and Nuclear Physics; the U.S. National Science Foundation; the Swiss National Science Foundation; the Science and Technology Facilities Council of the United Kingdom; and The Royal Society (United Kingdom). Additional support for 
the laser calibration system and cosmic ray tagger was provided by the Albert Einstein Center for Fundamental Physics (Bern, Switzerland).

\section{References}

[1] DUNE collaboration, The DUNE Far Detector Interim Design Report Volume 1: Physics, Technology and Strategies, arXiv: 1807.10334.

[2] MicroBooNE collaboration, Design and Construction of the MicroBooNE Detector, 2017 JINST 12 P02017 [arXiv: 1612 . 05824].

[3] MicroBooNE, LAR1-ND and ICARUS-WA104 collaborations, A Proposal for a Three Detector Short-Baseline Neutrino Oscillation Program in the Fermilab Booster Neutrino Beam, arXiv: 1503.01520.

[4] B.L. Henson, Mobility of positive ions in liquefied argon and nitrogen, Phys. Rev. 135 (1964) A1002.

[5] T. Dey and T. Lewis, Ion mobility and liquid motion in liquefied argon, J. Phys. D 1 (1968) 1019.

[6] Y. Li et al., Measurement of Longitudinal Electron Diffusion in Liquid Argon, Nucl. Instrum. Meth. A 816 (2016) 160 [arXiv: 1508.07059].

[7] A. Ereditato, I. Kreslo, M. Lüthi, C. Rudolf von Rohr, M. Schenk, T. Strauss et al., A steerable UV laser system for the calibration of liquid argon time projection chambers, 2014 JINST 9 T11007 [arXiv: 1406.6400].

[8] J. Sun, D. Cao and J. Dimmock, Investigating laser-induced ionization of purified liquid argon in a time projection chamber, Nucl. Instrum. Meth. A 370 (1996) 372.

[9] B. Rossi et al., A Prototype liquid Argon Time Projection Chamber for the study of UV laser multi-photonic ionization, 2009 JINST 4 P07011 [arXiv: 0906. 3437].

[10] I. Badhrees et al., Measurement of the two-photon absorption cross-section of liquid argon with a time projection chamber, New J. Phys. 12 (2010) 113024 [arXiv: 1011.6001].

[11] A. Ereditato, C.C. Hsu, S. Janos, I. Kreslo, M. Messina, C. Rudolf von Rohr et al., Design and operation of ARGONTUBE: a 5 m long drift liquid argon TPC, 2013 JINST 8 P07002 [arXiv: 1304.6961].

[12] https://amplitude-laser.com/produit/surelite-i-ii-iii/, (Accessed 08-06-2020).

[13] https://www.zaber.com/products/optical-mounts/T-OMG/specs, (Accessed 23-08-2019).

[14] http://www.continuumlasers.com, (Accessed 23-08-2019).

[15] https://www.altechna.com/products/watt-pilot-motorized-attenuator-enhanced-version/, (Accessed 23-08-2019).

[16] https://www.altechna.com/wp-content/uploads/2018/09/watt-pilot-user-manual-a4-full-7.2.pdf, (Accessed 23-08-2019).

[17] https://thermionics.com/product-category/manipulators/rnn-rotary-seals/, (Accessed 08-06-2020).

[18] MicroBooNE collaboration, Ionization electron signal processing in single phase LArTPCs. Part I. Algorithm Description and quantitative evaluation with MicroBooNE simulation, 2018 JINST 13 P07006 [arXiv: 1802 .08709].

[19] MicroBooNE collaboration, Ionization electron signal processing in single phase LArTPCs. Part II. Data/simulation comparison and performance in MicroBooNE, 2018 JINST 13 P07007 [arXiv: 1804.02583]. 
[20] E.L. Snider and G. Petrillo, LArSoft: Toolkit for Simulation, Reconstruction and Analysis of Liquid Argon TPC Neutrino Detectors, J. Phys. Conf. Ser. 898 (2017) 042057.

[21] MicroBooNE collaboration, The Pandora multi-algorithm approach to automated pattern recognition of cosmic-ray muon and neutrino events in the MicroBooNE detector, Eur. Phys. J. C 78 (2018) 82 [arXiv: 1708.03135].

[22] GEANT4 collaboration, GEANT4: A Simulation toolkit, Nucl. Instrum. Meth. A 506 (2003) 250.

[23] M. Mooney, The MicroBooNE Experiment and the Impact of Space Charge Effects, in Proceedings, Meeting of the APS Division of Particles and Fields (DPF 2015): Ann Arbor, Michigan, U.S.A., 4-8 Aug 2015, arXiv: 1511.01563.

[24] C. Jamin, S. Pion and M. Teillaud, 3D Triangulation Data Structure, in CGAL User and Reference Manual, 4.13 ed., CGAL Editorial Board, (2018).

[25] C. Jamin, S. Pion and M. Teillaud, 3D Triangulations, in CGAL User and Reference Manual, 4.13 ed., CGAL Editorial Board, (2018).

[26] S. Amoruso et al., Analysis of the liquid argon purity in the ICARUS T600 TPC, Nucl. Instrum. Meth. A 516 (2004) 68.

[27] W. Walkowiak, Drift velocity of free electrons in liquid argon, Nucl. Instrum. Meth. A 449 (2000) 288. 